\author{
MÉMOIRE DE MAÎTRISE \\ PRÉSENTÉ À \\ L'UNIVERSITÉ DU QUÉBEC À CHICOUTIMI \\ COMME EXIGENCE PARTIELLE
}

DE LA MAÎTRISE EN ÉTUDES ET INTERVENTIONS RÉGIONALES

PAR

ÉLOISE GAUDREAU

INTERPRÉTATIONS DE LA TENSION ENTRE PRINCIPES DE LUTTE ET PRATIQUE MILITANTE EN MATIÈRE DE RAPPORTS DE GENRE DANS LES ORGANISATIONS LIBERTAIRES : LE CAS DE L'UNION COMMUNISTE LIBERTAIRE (UCL) 


\section{NOTES AUX LECTRICES ET AUX LECTEURS}

La langue reflète les structures, préjugés et rapports de force inhérents à toute société. Ainsi, le «masculin épicène », qui est généralement utilisé, contribue à convoyer le sexisme et les inégalités, puisque, soi-disant pour alléger le texte ${ }^{1}$, on nous enseigne depuis notre plus jeune âge que le «masculin l'emporte».

Dans ce mémoire, je recoure à la féminisation linguistique. En vertu des positions idéologiques que je défends, il est logique que je refuse de considérer que le masculin se confonde avec l'universel et corresponde à un usage « neutre». La langue est politique. La féminisation peut contribuer à rendre visible les femmes et à reconnaître leur contribution au travail politique.

Par conséquent, dans ce mémoire, le recours au masculin (tant au singulier qu'au pluriel) indique qu'il n'est question que de personnes de sexe masculin. Le recours au féminin signifie qu'il est question de personnes de sexe féminin. La forme féminisée à l'aide d'un tiret (ex : militant-e-s) renvoie aux deux sexes, tout comme la féminisation dite « au long » (ex : militants et militantes).

\footnotetext{
${ }^{1}$ L'Académie française résiste à la fẻminisation en invoquant la lourdeur du texte. Or, dans «Féminisation et lourdeur du texte », Pascal Gygax a démontré que la féminisation des titres professionnels n'entravait pas la vitesse de lecture : les lectrices et lecteurs s'habituent dès la seconde occurrence de titre féminisé.
} 


\section{RÉSUMÉ}

Ce mémoire présente une analyse de l'articulation des principes féministes et des pratiques militantes dans les organisations libertaires. Afin de déterminer les termes dans lesquels les militants et militantes l'interprètent, 13 entrevues ont été réalisées avec des militant-e-s de l'UCL (l'Union Communiste Libertaire).

La pertinence de cette recherche repose sur trois éléments. Premièrement, la littérature portant sur les rapports de pouvoir genrés au sein du militantisme mixte s'intéresse surtout aux partis politiques, syndicats et mouvements sociaux, alors que les recherches portant sur le milieu libertaire font rarement du genre leur entrée principale. Par ailleurs, la plupart des analyses étudient le contexte français, où la culture politique militante et le contexte sociohistorique modulent les rapports de genre de façon différente. De plus, la reproduction des rapports de pouvoir genrés en dépit d'une volonté explicite de les subvertir n'a été que très peu explorée, et demeure une question ouverte, tant sur le plan politique que théorique.

Dans la vie militante, les rapports de pouvoir genrés s'articulent principalement autour de l'enjeu de la division sexuelle du travail: invisibilisation du travail des femmes, appropriation des tâches rétribuables par les hommes, sous-représentation médiatique des femmes, disparité (quantitative et qualitative) en matière de prise de parole. Ces situations semblent en contradiction avec les idéaux des libertaires qui cherchent à combattre tous les systèmes d'oppression, tant au niveau de la société que de leurs rapports quotidiens au sein de leurs collectifs.

Les lieux et modalités de la reproduction des rapports de pouvoir permettent de supposer que la mouvance libertaire constitue bel et bien un mouvement social sexué (Kergoat, 1992, 2001 et 2010, Dunezat, 1998, 1999 et 2007). Est-ce que les militant-e-s perçoivent cette sexuation? Et l'interprètent-ils et elles en termes de tension, de contradiction, de cohérence?

Or, cette recherche montre que les militant-e-s de l'UCL n'interprètent pas le rapport entre principes égalitaires et pratiques inégalitaires en termes de contradiction. Ce terme recouvre mal une réalité beaucoup plus complexe, dont cette recherche tentera de rendre compte. Je montrerai que c'est l'idée de la tension qui est la plus pertinente pour illustrer le jeu dialectique et dynamique qui s'élabore entre les principes et les pratiques. Ceux-ci sont constamment réévalués et modifiés, à travers des débats, l'introduction, la modification ou la suppression de stratégies de gestion des rapports de pouvoir. Grâce à ces efforts et stratégies, les militant-e-s sentent que leur organisation devient de plus en plus égalitaire grâce à cette force de travail mise en œuvre pour cheminer vers une cohérence toujours plus grande.

Les militant-e-s identifient les facteurs qui font en sorte que la tension est maintenue. D'une part, il n'y a pas de consensus, à l'UCL, sur ce qui constitue un rapport de pouvoir. L'UCL doit composer avec les définitions en termes d'agressivité et les définitions plus 
systémiques (en termes d'inégalité de position entre groupes sociaux). La coexistence de plusieurs définition complique l'identification des situations qui constituent des rapports de pouvoir. En même temps, les militant-e-s reconnaissent les efforts individuels et collectifs qui sont mis en œuvre pour modifier les rapports de genre. Cet optimisme est cependant tempéré par la conscience de l'inégalité des motivations envers la subversion des rapports de genre; des stratégies sont ridiculisées et banalisées par certains militants et de ce fait, elles semblent avoir moins d'effets.

Cette tension entre principes et pratiques, entre stratégies et résultats est interprétée de diverses façons. D'une part, les libertaires rencontré-e-s ne recourent pas à des explications essentialistes, préférant les explications qui renvoient aux structures sociales patriarcales et aux effets de la socialisation différenciée, notamment en matière de socialisation politique. Les femmes militantes, surtout, imputent la reproduction des rapports de pouvoir genrés à une dynamique interne oppressive envers les femmes. 


\section{REMERCIEMENTS}

Mes premiers remerciements s'adressent à deux personnes qui ne soupçonnaient pas qu'en répondant à mon (trop) précoce «Maman, Papa, c'est quoi la société? », elles ouvriraient la porte à une infinie quête du savoir dans le champ des sciences sociales. Donc, merci à mes parents, sans qui je n'aurais sans doute pas développé cette curiosité, cette exubérance intellectuelle et ce désir de la performance (pour le meilleure et pour le pire).

Comment, ensuite, ne pas remercier ma directrice, Marielle Tremblay, et mon codirecteur, Pierre-André Tremblay? Vous m'avez laissée assez de liberté pour produire un mémoire qui me ressemble et dont je suis fière. Par ailleurs, je vous suis reconnaissante d'avoir tempéré mes ardeurs, rassuré mes craintes, orienté mes errances et d'avoir cru en mon projet. En plus de vos scientifiques conseils, j'ai pu tirer profit du plaisir de longues discussions. Merci également pour les nombreuses relectures et les conseils de dernière minute.

À l'Union communiste libertaire, mes plus solidaires remerciements : cette recherche n'aurait pu voir le jour sans la confiance que vous m'avez accordée et sans l'intérêt des militants et militantes qui ont accepté d'y participer. Merci aussi d'être là, et de défendre avec fougue la possibilité d'un monde: radicalement différent.

Enfin, je ne saurais passer sous silence la contribution cruciale de toutes mes collègues et amies du H5-1340 : Caroline Jean, Josie-Ann Bonneau et Mylène Geoffroy, avec qui j'ai partagé tellement, tellement plus que des idées.

Un «merci » en lettres majuscules à Stéphane Turcotte et à Geneviève Larouche pour m'avoir orientée vers cet objet de recherche et pour toutes les discussions informelles sur le sujet. À Mathieu Bisson pour les échanges académiques, sérieux et pas sérieux. Enfin, à David, qui a vécu avec moi les hauts, mais surtout les bas de mon mémoire : merci pour la présence, le support et les lunches. 


\section{TABLE DES MATIÈRES}

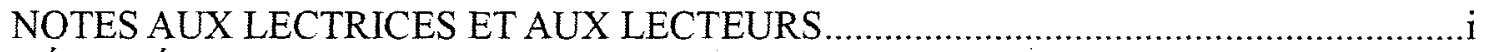

RÉSUMÉ

REMERCIEMENTS ........................................................................................

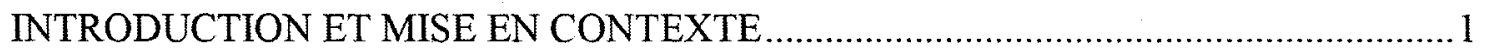

Mise en contexte : la mouvance libertaire contemporaine ...........................................

CHAPITRE 1 PROBLÉMATIQUE ET ÉLÉMENTS THÉORIQUES .............................10

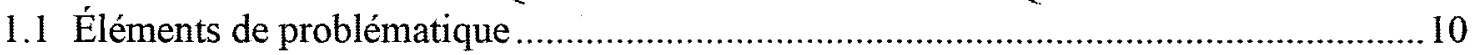

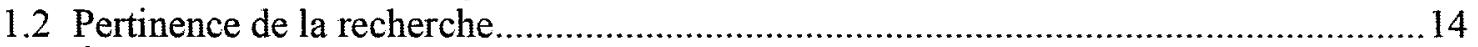

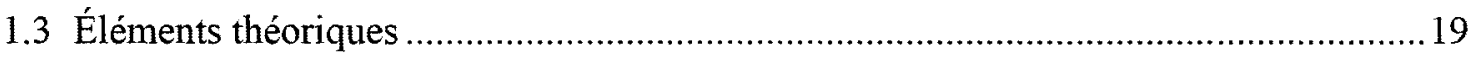

1.3.1 Rapports de genre et rapports de domination ................................................19

1.3.2 Positions sociales et positionnalités..................................................................22

1.3.3 Des rapports de pouvoir genrés?..............................................................2

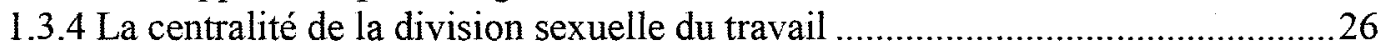

1.3.5 Les mouvements sociaux sexués ………………………………....................30

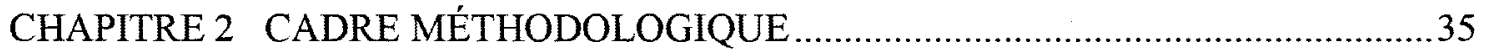

2.1 Approche féministe, épistémologie, rapport sujet-objet et standpoint theory ..............36

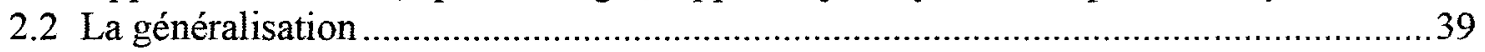

2.3 L'étude de cas comme stratégie de recherche ……................................................40

2.4 Le cas à l'étude : l'union communiste libertaire (UCL) ................................................41

2.5 Technique de collecte de données : entrevues semi-dirigées .......................................43

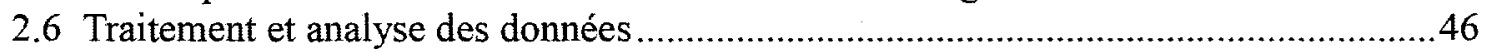

CHAPITRE 3 L'ARTICULATION DES PRINCIPES ET DES PRATIQUES EN

MATIÈRE DE RAPPORTS DE GENRE : «L'AMBIVALENCE NORMATIVE » .........48

3.1 Les principes centraux sont d'abord anticapitalistes ................................................49

3.1.1 Principes associés à la mouvance libertaire et à l'UCL .....................................50

3.1.2 Les motivations individuelles à l'adhésion à l'UCL .........................................51

3.1.3 Les militant-e-s présentent divers degrés de féminisme …………………........52

3.2 L'ambivalence normative : l'écart entre les principes et les pratiques : ........................53

3.2.1 Organisation pro-féministe et sous-représentation des femmes........................54

3.2.2 Le principe de rotation des tâches et la division sexuelle du travail

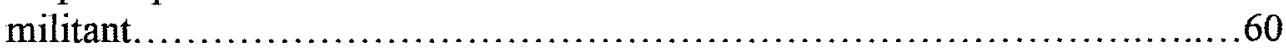

3.2.3 Principes d'équité dans la prise de parole et pratiques discursives oppressives 67

3.2.4 Triple oppression, anarcha-féminisme et hiérarchisation des luttes................... 74

3.2.5 Le principe du " privé est politique » et les rappports de pouvoir dans les espaces informels 
CHAPITRE 4 LES RESSOURCES EXPLICATIVES, OU LES EXPLICATIONS DE LA

TENSION ENTRE PRINCIPES ET PRATIQUES

4.1 Le rapport entre principes et pratiques s'exprime surtout en termes de « tension » qu'en termes de contradiction.

4.2 Les diverses définitions des rapports de pouvoir et leurs impacts sur l'évaluation du

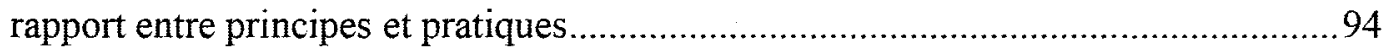

4.3 Des défis liés à l'identification des rapports de pouvoir dans la pratique ...................97

4.4 Par conséquent, l'UCL est définie comme espace égalitaire..................................... 101

4.5 Les défis liés à l'intervention sur les rapports de pouvoir ...................................... 103

4.5.1 L'intervention pour annihiler les rapports de pouvoir genrés: une force de travail qui s'incarne dans des stratégies .............................................................. 104

4.5.2 Aborder les rapports de pouvoir genrés .................................................110

4.6 Réduire la tension entre principes et pratiques? ................................................113

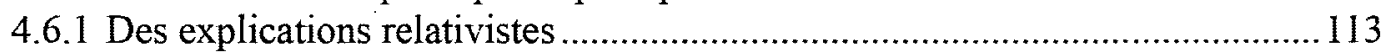

4.6.2 des améliorations à approfondir ........................................................ 116

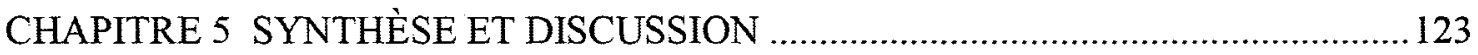

5.2 La socialisation et les structures sociales patriarcales ....................................... 128

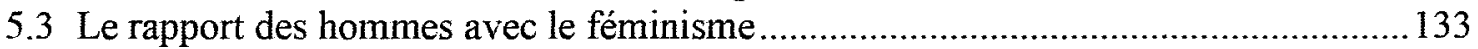

5.4 Une culture militante masculine ... même dans le milieu libertaire...........................136

5.5 L'individualisation de l'analyse des rapports de pouvoir .................................... 140

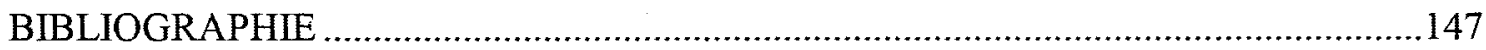

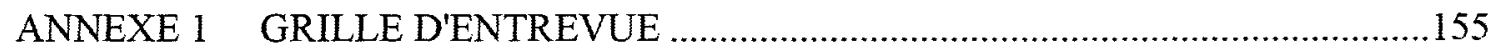

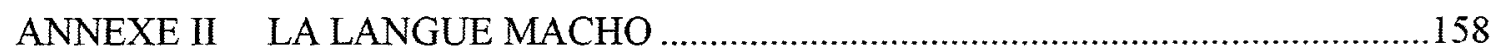

ANNEXE III LISTE DES THÉMATIQUES DU JOURNAL CAUSE COMMUNE .....161

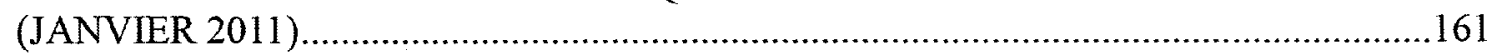




\section{INTRODUCTION ET MISE EN CONTEXTE}

Plusieurs ouvrages mettent en lumière les processus par lesquels les rapports de pouvoir genrés se déploient et se reproduisent dans les activités militantes (Roux et Filleule, 2009), que ce soit dans les partis politiques, les syndicats ou les mouvements sociaux. Les chercheuses et chercheurs y identifient des mécanismes qui contribuent à la construction de positions inégalitaires : division sexuelle du travail militant, invisibilisation du travail des femmes, appropriation des tâches rétribuables ${ }^{2}$ par les hommes, sous-représentation médiatique des femmes, etc. ${ }^{3}$

Cette question m'apparaît particulièrement intéressante au sein des collectifs libertaires québécois, dont le discours et les principes de lutte soulignent l'importance d'articuler la lutte au capitalisme à la lutte au Patriarcat (Dupuis-Déri, 2010). Recourant à des formes organisationnelles qui visent l'éradication des rapports de domination, dont ceux basés sur le genre (Dupuis-Déri, 2009; Kruzynski et Sévigny, 2005), la pratique militante demeure néanmoins empreinte d'une différenciation traditionnelle des rôles, statuts et pouvoirs des personnes en fonction de leur genre (Roux, 2009). Cette étude a pour objet l'articulation entre les principes de lutte (la volonté officielle, le discours) et la reproduction des rapports de pouvoir genrés.

En effet, cette recherche vise à saisir les interprétations que font les militants et les militantes de la contradiction apparente entre le discours antisexiste et des pratiques militantes qui vont dans le sens opposé. Autrement dit, je m'intéresse à l'analyse qui est

${ }^{2}$ La notion de «tâches rétribuables » telle qu'utilisée par les auteur-e-s fait référence aux tâches qui donnent accès à des rétributions sociales et symboliques (reconnaissance et valorisation, augmentation du «capital militant "). Cette rétribution symbolique peut impliquer des tâches qui préparent un accès à des situations professionnelles, à des postes à responsabilités, qui donnent accès à un réseau élargi de relations (Bargel, 2005).

${ }^{3}$ Voir ces auteur-e-s : Bargel, 2005; Blais, 2008, Drapeau, 2007; Dupuis-Déri, 2009; Dunezat, 1998, 2004, 2007; Falquet, 1998; Filleule 2008; Landry, 2007; Monnet, 1998, Quirion, 2008 ; Roux, 2009, Tratt; 2006 
faite de l'articulation de deux normes sociales contradictoires: celle de l'égalité et celle de la division sexuelle du travail/statut/pouvoir (Roux, 2009). Je cherche à identifier les discours mis en place par les militants et les militantes libertaires pour analyser la sexuation de leurs collectifs et organisations, afin de saisir les obstacles ultimes à l'instauration de rapports égalitaires, lorsque ni l'idéologie essentialiste ou l'idée du " grand soir »" ne peuvent être invoquées.

Le premier chapitre du mémoire expose la problématique et les éléments théoriques. J'y présente l'objet d'étude, les questions de recherche, ainsi que la pertinence scientifique et sociale. Dans la deuxième partie, je détaille mon cadre théorique. $J$ 'y expose les intuitions fondamentales qui ont guidé mon analyse, soit la force du genre en tant que structure sociale, la notion de mouvement social sexué et la question du poids épistémologique du vécu. J'appelle par ailleurs à concevoir les rapports de genre comme des rapports de pouvoir, lesquels comportent une dimension structurelle et une dimension relationnelle, à la fois position sociale et assignation à ces positions (Anthias, 1998, Grolleau et Kruzynski, 2007). Je placerai ensuite ces concepts dans le cadre militant, afin d'y souligner le caractère central de la division sexuelle du travail. La permanence de cette division sexuelle du travail dans les organisations militantes m'amène à les considérer sous l'angle des mouvements sociaux sexués (Kergoat, 1992, Dunezat, 1998 et 1999).

Le second chapitre fait état de mon cheminement méthodologique. Cette recherche s'appuie sur une étude de cas, qui compte treize entrevues, réalisées auprès de personnes militant au sein de deux collectifs locaux de l'Union des communistes libertaires (UCL). De plus, des précisions contextuelles sur les contours théoriques de la notion d'《anarchie » ou de «libertaire » sont proposées afin d'en souligner les principes centraux et les formes organisationnelles qui y sont favorisées.

\footnotetext{
${ }^{4}$ L'idée du « grand soir » fait référence au moment de la rupture révolutionnaire, qui marquerait la fin entre un ordre ancien et un ordre nouveau. Pendant longtemps, les groupes de gauche et d'extrême-gauche (communistes, anarchistes, socialistes) ont évacué les critiques des femmes insatisfaites de leur position subordonnée dans le mouvement en arguant que la révolution créera un monde égalitaire.
} 
L'analyse de mes données m'a permis d'identifier certaines zones de tension entre les principes et les pratiques. Il me fallait effectivement confirmer l'existence et les modalités des rapports de pouvoir genrés, et ainsi confirmer mes attentes de recherche issues de la littérature. Ces tensions, qui sont présentées au troisième chapitre, concernent la sousreprésentation quantitative des femmes, la division sexuelle du travail, les rapports de pouvoir dans la sphère informelle et la hiérarchisation des luttes qui entrent en contradiction, ou en tension, avec certains principes chers au milieu libertaire et à l'UCL : la rotation des tâches, la rotation des prises de parole, la reconnaissance de la triple oppression $^{5}$ ou que le privé est politique.

Le quatrième chapitre discute des interprétations militantes de ces rapports de pouvoir genrés. Ainsi, une variété de définitions des rapports de pouvoir se conjugue aux défis de repérer et nommer les rapports de pouvoir dans la pratique militante. Par ailleurs, les personnes interviewées identifient les difficultés rencontrées dans la mise en cuvre de stratégies pour lutter contre les rapports genrés, puisque le fait d'adopter des principes féministes ne se traduit pas nécessairement par un comportement égalitaire en l'absence de «force de travail » pour éliminer les hiérarchies et cheminer vers l'égalité. Enfin, je présente les interprétations des «résultats », c'est-à-dire les améliorations observées en matière de rapports de genre et l'identification du travail qu'il reste à faire.

Pour terminer, je présenterai une discussion sur les interprétations qui apparaissent en filigrane des entrevues, et qui permettent d'expliquer, en ultime instance, la reproduction des rapports de pouvoir genrés dans les organisations libertaires. Il appert que ce phénomène s'avère complexe, et constitue le produit de plusieurs causes qui s'alimentent mutuellement. Ainsi, la socialisation genrée, l'impact des structures sociales inégalitaires, le rapport particulier des hommes au féminisme, la persistance d'une culture militante masculine, la dynamique organisationnelle ainsi que les rapports interindividuels inégaux se combinent pour teinter la façon particulière dont opèrent les rapports de genre dans une

\footnotetext{
${ }^{5}$ La triple oppression est une analyse qui reconnaît trois systèmes d'oppression interdépendants : le
} capitalisme, le Patriarcat et l'État. 
organisation.

\section{MISE EN CONTEXTE : LA MOUVANCE LIBERTAIRE CONTEMPORAINE}

Définir le concept de «collectifs militants libertaires » n'est pas chose aisée. D'une part, cela implique la sélection d'une étiquette conceptuelle qui ne fera pas l'unanimité des militant-e-s. D'autre part, la gauche radicale/anarchiste, qui s'avère un milieu diversifié sur le plan des objectifs et des pratiques, ne représente pas un objet clairement identifiable et bien délimité (Luck, 2008; Gagnon et Rioux, 1998). Il demeure toutefois possible de penser « la gauche de la gauche » comme un « tout » en s'attachant aux similitudes en matière de principes et de formes organisationnelles (celle du « collectif $»)$ et, ainsi, de penser ce « tout » comme représentant la « mouvance libertaire ».

Plusieurs désignations analogues coexistent (libertaires, radicaux, anarchistes, autogestionnaires, frange radicale, autonomie collective, antiautoritaire). Sur le plan de la pensée politique, il est possible d'établir des distinctions théoriques et historiques entre libertaires et anarchistes, lesquels, pour plusieurs, renvoient aux anarchistes de la fin du $19^{\mathrm{e}}$ siècle plutôt qu'à ceux de la mouvance altermondialiste post-Seattle ${ }^{6}$ (Baillargeon, 2005; Dupuis-Déri, 2010). Or chez Kruzynski (2005) comme chez Luck (2008), les deux termes sont utilisés de façon relativement indistincte, bien que certain-e-s militant-e-s refusent l'une ou l'autre de ces étiquettes ${ }^{7}$. Le terme « anarchiste » étant généralement connoté négativement (Baillargeon, 2005), je recourrai au terme « libertaire », qui me semble plus approprié, parce qu'il trace bien la différence historique entre les anarchistes post-Seattle et les autres.

Le milieu libertaire se caractérise par une variété de tactiques, de pratiques, d'objectifs, de

\footnotetext{
${ }^{6}$ La contestation de Seattle (1999) marque un point tournant dans la constitution du mouvement altermondialiste et de sa frange plus radicale, la mouvance libertaire. Au Québec, lanarchisme, bien que présent dans les années 1990 , a effectué un retour sur la scène politique, surtout à l'occasion du contresommet de Québec en avril 2001 (Dupuis-Déri, 2009).

7 « Moi, je suis pas anarchiste, je suis communiste libertaire! » (dit un militant dans une discussion informelle). Une autre militante refusait le concept d'autogestion, puisqu'il lui semblait référer à l'idée de « gestion » qui la dégoûtait.
} 
projets (Dupuis-Déri, 2009) : espaces de vie libérés, projets collectifs, émissions de radio, groupes d'action directe, désobéissance civile, manifestations, éducation populaire, etc., dans le but de bloquer des institutions ou construire des alternatives exemptes de rapports de domination (Kruzynski et Delisle-L'Heureux, 2007). Il couvre une pluralité de thématiques: antiracisme, écologie radicale, féminisme, libération de prisonniers politiques à l'étranger, justice migrante, etc.

De plus, les divers groupes libertaires ne présentent pas d'unité en matière de référents théoriques, puisque, contrairement aux marxistes, les libertaires ne réfléchissent pas en termes théoriques ou philosophiques. ils et elles proposent plutôt une réflexion éthique à propos de la pratique et des moyens de l'organisation (Graeber, 2004).

Pour cette raison, les référents théoriques sont variés et empruntent à divers courants : coopérativisme, insurrectionisme, anarcho-syndicalismes, anarcho-communismes, plateformisme, marxisme (Baillargeon, 2005; Graeber, 2004; Schmidt et Van der Walt, 2009).

Les « principes de lutte » représentent les idéaux et idées fondamentales du collectif, qui sont formalisés dans le discours et qui encadrent, d'une certaine façon, la conduite idéale des militant-e-s et déterminent le type d'action collective menée. Ces principes organisationnels sont constitués d'idées, de valeurs et de positions politiques qui composent le fondement du collectif. Par ailleurs, il s'agit d'éléments du discours public qui révèlent la façon dont l'organisation souhaite être perçue. Ces idées fondamentales sont le noyau de l'organisation, celui avec lequel les personnes qui souhaitent s'impliquer doivent concorder, souvent sous peine d'expulsion.

Breton et Kruzynski (2010) font référence à une «culture politique libertaire » qui permettrait d'unir des collectifs aux tactiques et objectifs divers sous une même chape conceptuelle. Cette culture anime des groupes qui se rangent généralement derrière des valeurs antiautoritaires et anticapitalistes, et valorisent la liberté, la solidarité et l'égalité 
(Baillargeon, 2005; Breton et Kruzynski, 2010; Dupuis-Déri 2010; Luck, 2008).

À cet égard, les libertaires rejettent les formes de pouvoir considérées illégitimes (le « pouvoir sur ») (Baillargeon, 2001), tout comme les hiérarchies et les systèmes d'oppression (capitalisme, racisme, sexisme). Par ailleurs, les libertaires critiquent la logique partisane électoraliste des partis politiques (Luck, 2008). Ils et elles considèrent que la véritable démocratie est anarchiste et non libérale, c'est-à-dire qu'elle est l'affaire de toutes et tous, et non seulement d'une élite, et qu'elle doit faire l'objet d'un processus ouvert et égalitaire permettant la participation (Graeber, 2005).

Par ailleurs, les libertaires ne se limitent pas aux manifestations de l'oppression, mais à ce qui est analysé comme en constituant les racines, les causes profondes.

Ainsi, malgré une apparente diversité, tous ces groupes sont " des mouvements que l'on peut qualifier de libertaires en raison de leurs modes d'organisation et d'action " (Luck, 2008: 131). Cela signifie que les libertaires recourent à des formes organisationnelles particulières, où les fins (principes) doivent transparaître dans les moyens utilisés (pratiques et formes organisationnelles) pour s'inscrire dans « une très longue tradition anarchiste selon laquelle les organisations militantes devraient incarner les principes de l'anarchisme » (Dupuis-Déri, 2009: 198).

Cette forme organisationnelle est celle du «collectif $»$ : elle repose sur des principes égalitaires et horizontaux, où il y a absence de leadership formel, de «postes » assignés, de tâches réservées. L'idée d'autogestion suppose l'absence de distinction entre la base et l'organisation dans les processus de prise de décision, qui se veulent égalitaires, participatifs, délibératifs et consensuels (Piotte, 2007; Graeber, 2005). L'assignation des tâches vise à ce que tous et toutes puissent se partager alternativement les responsabilités qui auront été déterminées par elles et eux (Drapeau, 2007). 
Le « collectif » désigne une association volontaire de personnes qui partagent des objectifs communs et s'étant dotées d'une visibilité et d'une identité publique (un nom, un logo, etc.) et ayant mis en place des procédures de coordination interne (réunions périodiques, moyens de communication collectifs) (Luck, 2008: 27). L'avantage de cette définition est qu'elle utilise les pratiques organisationnelles comme éléments de définition, ce qui permet de prendre en compte les groupes informels que sont souvent les collectifs. En effet, cette acception de l'« organisation »n'implique pas nécessairement un statut juridique ou des règles formelles gouvernant l'adhésion individuelle.

En somme, ce sont « ces modes d'organisation et de prise de décision [qui] constituent des éléments de convergence, un facteur de cohérence au sein d'une nébuleuse relativement hétérogène politiquement » (Luck, 2008: 41).

Plusieurs auteur-e-s libertaires offrent des points de vue divergents à propos du degré de convergence entre le féminisme et l'anarchisme. Or, comme il a été exposé ici, cette communauté de lutte n'a rien d'automatique, vu la persistance de rapports de pouvoir qui affectent tant les priorités stratégiques de l'organisation que le statut des femmes dans celleci.

L'anarcha-féminisme suppose une communauté de luttes entre le féminisme et l'anarchisme (Baillargeon, 2001). Or, c'est le sexisme de la pensée anarchiste, ou à tout le moins, l'invisibilisation des "questions femmes » qui a animé le travail et la pensée d'Emma Goldman, et inspiré ce courant de l'anarchisme. L'anarcha-féminisme est une réponse aux anarchistes qui justifient les rapports sociaux de sexe inégaux ou qui considèrent que la transformation des rapports de genre est une question «à côté » ou «moins importante » que la lutte contre l'État et le capitalisme.

Selon certain-e-s, lutter contre toutes les dominations et les oppressions signifie également la lutte contre la domination des hommes sur les femmes (Brown, 1993), mais cette 
articulation ne va pas de soi, étant donné la prégnance du «machisme anarchiste » (Monnet, 2001). Dans les faits, au Québec, plusieurs collectifs libertaires se positionnent en faveur du féminisme et dénoncent le Patriarcat, le sexisme, l'hétéronormativité et l'homophobie (Dupuis-Déri, 2010). Certains groupes libertaires sont plus explicitement organisés autour du principe de l'«anarcha-féminisme » (Baillargeon, 2001), pour marquer la rupture avec une tradition anarchiste considérée comme sexiste. Par exemple, Proudhon, à qui est attribué l'introduction de l'appellation « anarchisme » (Baillargeon, 2001; Luck, 2008), est considéré comme un misogyne notoire (Dupuis-Déri, 2010).

D'autres récusent l'idée que la tradition anarchiste ait été (et soit) sexiste : l'oppression des femmes n'y aurait pas nécessairement été assimilée à l'oppression du capitalisme et plusieurs auteurs auraient pris en compte les rapports de genre (Schmidt et Van der Walt, 2009). Il est vrai que d'autres «penseurs barbus » ont critiqué l'état des rapports hommesfemmes, notamment Bakounine, qui a dénoncé le manque de cohérence de certains de ses contemporains entre les idées égalitaires présentées dans leurs écrits et leur attitude dans la sphère domestique (Zabalazza).

The movement's perspective on women's emancipation followed a similar logic of trying to find a means of linking struggle against the specific problems faced by women within the larger project of revolutionary class struggle. In line with its commitment to economic and social inequality as well as individual freedom, the anarchist tradition aimed to create equality between the sexes as part of its project of creating a new society (Schmidt and Van der Walt, 2009: 324)

Pour leur part, les féministes qui s'impliquent dans le milieu anticapitaliste dénoncent la centralité du capitalisme comme système unique ou prioritaire pour l'explication des rapports de pouvoir (Blais et Courcy, 2010). Pour cette raison, la question de la persistance du sexisme et des rapports de pouvoir genrés m'apparaît intéressante. Selon moi, il est nécessaire d'explorer cette contradiction, pour aller au-delà de l'idée que «la couleur politique ne change rien aux comportements de domination des hommes » (Monnet, 2001: 468). 
Il convient de souligner la variété des féminismes dans le milieu libertaire (Breton et coll., 2007). Pour les collectifs retenus pour cette recherche, c'est surtout le féminisme dit «radical» qui fait office de base de réflexion. Appartenant à la «deuxième vague » féministe, ce type de féminisme s'organise sur la base de la non-mixité femme, et tient un discours anticapitaliste et anti-patriarcal. L'oppression des femmes y est étroitement associée à l'oppression économique, et centrée sur la division du travail. Cette posture sera abordée plus avant dans mon cadre théorique, puisque c'est l'angle d'analyse que j'ai retenu. Par contre, d'autres tendances du féminisme influencent le milieu libertaire québécois et les collectifs que j'ai retenus pour ce mémoire. Les réflexions issues du black feminism, ou du women of colour feminism ont nourri une réflexion qui se veut plus intersectionnelle, c'est-à-dire qui prend en compte plusieurs rapports sociaux de domination dans son analyse, d'où le principe de triple oppression que j'ai abordé plus tôt. Bien que. plus ancrée dans le milieu libertaire anglophone, la réflexion queer radical enrichit les réflexions et la pratique, en critiquant l'identité sexuelle, thématique jugée négligée par les féministes radicales (Breton et coll., 2007). Ces féministes critiquent la non-mixité femme et proposent une non-mixité féministe, et s'attaquent aux questions d'hétéronormativité tout en critiquant l'identité sexuelle. 


\section{CHAPITRE 1}

\section{PROBLÉMATIQUE ET ÉLÉMENTS THÉORIQUES}

\section{1 ÉLÉMENTS DE PROBLÉMATIQUE}

Cette recherche porte sur l'articulation entre les principes de luttes et la pratique militante, en matière de rapports sociaux de sexe, dans les organisations militantes de la frange libertaire. Elle vise à saisir la façon dont les militantes et militants interprètent et analysent la confrontation de deux normes sociales antagonistes : l'idée d'égalité portée par la pensée libertaire contemporaine et la division sexuelle du travail qui y est (re)produite (Roux, 2009).

C'est cette apparente contradiction qui constitue le problème de recherche. D'une part, le fait que les libertaires s'attachent à lutter contre toutes les formes de domination et d'oppression, dont, en principe, celle des hommes sur les femmes (Dupuis-Déri, 2009; Monnet, 2001) et qu'ils recourent à des formes organisationnelles favorisant l'horizontalité pourrait porter à présumer que les rapports entre les hommes et les femmes s'y articuleraient différemment.

Pourtant, la littérature portant sur l'état des rapports de pouvoir au sein du militantisme mixte (partis politiques, syndicats, mouvements sociaux, projets alternatifs) fait état d'inégalités entre les « sexes » et de domination des hommes sur les femmes ${ }^{8}$. Les collectifs anarchistes, malgré leurs principes anti-oppression et leurs formes organisationnelles

${ }^{8}$ Voir ces auteur-e-s : Bargel, 2005; Blais, 2008, Drapeau, 2007; Dupuis-Déri, 2009; Dunezat, 1998, 2004, 2007; Falquet, 1998; Filleule 2008; Landry, 2007; Monnet, 1998, Quirion, 2008 ; Roux, 2009, Tratt; 2006 
horizontales, n'échappent pas à cette domination. En effet, «si les organisations militantes anarchistes actuelles se déclarent généralement antisexistes, la misogynie en parole et en acte y sévit tout de même » (Dupuis-Déri, 2009: 188) : division sexuelle du travail militant, permanence des normes de genre, esthétique militante viriliste (Dupuis-Déri, 2010), sousreprésentation des femmes (Luck, 2008) et invisibilisation du travail de celles-ci. Les auteur-e-s qui s'intéressent aux libertaires traitent presque inévitablement de l'état des rapports de genre dans ces collectifs, et les articulent en termes de pouvoir (Drapeau, 2007; Luck, 2008; Kruzynski et coll., 2007; Monnet, 2001; Quirion, 2008).

Par conséquent, il appert que le «machisme anarchiste» ou l'«anarchosexisme» constituent bel et bien des paramètres de la mouvance «antiautoritaire » (Dupuis-Déri, 2010; Monnet 2001; Thiers-Vidal, 2002) :

Pour une anarchaféministe, anarchisme et féminisme sont des synonymes, mais on ne peut s'arrêter à dire cela. Car les anarchistes ne l'entendent pas de cette façon. Elles/ils luttent contre toutes les formes de hiérarchie et de domination afin de les éliminer de nos relations sociales et politiques. En théorie, done l'anarchisme inclut la critique de la domination masculine, mais encore faut-il la reconnaître. En réalité, la mouvance respecte et reconduit les lois patriarcales, d'où une mouvance largement constituée d'hommes, blancs, et hétérosexuels (Monnet, 2001; 468-469)

En somme, cette apparente contradiction entre les principes de luttes (importance théorique de lutter contre l'oppression patriarcale) et la pratique militante interpelle la question du rapport entre les fins et les moyens de l'action, mais surtout du rapport entre les principes de luttes et les pratiques militantes, en matière d'antisexisme, de féminisme et de rapports de genre.

L'objectif de cette recherche est d'explorer les interprétations militantes de la confrontation de la norme d'égalité et la (re) production de la division sexuelle du travail, tant dans le cadre militant que non militant.

Les questions de recherche de ce mémoire s'inspirent des questionnements de Patricia Roux 
(2009), qui s'intéresse à la confrontation du discours d'égalité entre les hommes et les femmes à la persistance de la division sexuelle du travail.

Principalement, je cherche à savoir : comment les militantes et les militants de collectifs libertaires interprètent-ils et elles l'articulation des principes de lutte au sexisme et une pratique militante caractérisée par la reproduction de rapports de pouvoir basés sur le genre? Quels raisonnements mettent-ils et elles en place pour expliquer l'écart entre leurs expériences quotidiennes et les principes d'égalité valorisés? (Roux, 2009: 111).

Autrement dit: à quel point les personnes militantes s'accommodent-elles ou pas des logiques patriarcales du militantisme, c'est-à-dire des rapports de pouvoir qui sont contraires aux principes de luttes? Comment, selon elles, s'articulent les principes antisexistes et la pratique militante?

Plus précisément, je me demande :

- Les personnes militantes perçoivent-elles du sexisme, des rapports de pouvoirs ou plutôt une égalité au sein de leur pratique militante?

- Quels sont les lieux où une contradiction/tension est perçue et comment est interprétée cette contradiction/tension?

- Et finalement quelles analyses sociologiques peuvent être tirées de ces explications militantes?

Ces questions me permettront d'atteindre les objectifs de cette recherche. Celle-ci vise à explorer les explications mobilisées par les militant-e-s pour gérer l'articulation entre un discours de lutte au sexisme et une pratique militante empreinte de rapports de pouvoir basés sur le genre. Je souhaite dégager des éléments explicatifs qui me permettront de mieux comprendre le point de vue des actrices et acteurs sociaux engagé-e-s dans la subversion des rapports sociaux de sexe. Je cherche également à identifier les obstacles à la transformation sociale, en me basant sur les interprétations et représentations des militantes 
et militants libertaires. Si d'une part, le genre possède une dimension structurelle qui oriente les personnes (par la socialisation, notamment), celles-ci sont néanmoins dotées d'une capacité de distanciation critique qui leur permet de réaménager leurs rapports avec les autres. Autrement dit, les personnes militantes interrogées réfléchissent-elles plutôt en termes de structure ou en termes d'agencéité? Quelle place accorder au « genre en tant que structure sociale » (Risman, 2004) qui détermine les individus, quelle place accorder aux approches du positionnement social à travers l'interaction (Anthias, 1998; Grolleau et Kruzynski, 2007)?

$\grave{A}$ cet égard, je m'efforcerai d'articuler deux types d'explication: une qui repose sur l'acteur/actrice et sa position sociale, et une qui fait référence au poids du genre en tant que structure sociale. Je tenterai d'illustrer que « les pratiques et les discours militants n'obéissent pas au simple ordre de la reproduction sociale : la mobilisation est un processus qui contribue à fabriquer la sexuation du social, mais aussi un rapport à cette sexuation. » (Dunezat, 2007: 269).

C'est ce que cette recherche propose d'explorer : quel rapport à la sexuation est développé par les interviewé-e-s, et ultimement, voir si, et comment, le rapport à cette sexuation est lui-même genré.

Ainsi, j'aborderai la question du genre en tant que structure sociale. En ce sens, si les militantes et militants échouent à contrer les rapports de pouvoir genrés qui se situent au niveau interindividuel, il faut prendre conscience que les rapports de genre ne se déploient pas que dans les ramifications sociales, où, peut-être, le pouvoir est le plus visible (Foucault, 1976). Le genre est inscrit dans les institutions, dans la pratique politique et s'incarne dans des structures d'inégalités; le genre fait partie de la vie sociale, détermine les possibilités d'action des agents et leurs options de vie (Risman, 2004). L'analyse proposée dans ce mémoire relativise les « motivations de l'acteur-actrice » pour mettre en lumière le poids des socialisations primaire et secondaire en tant que processus visant l'apprentissage 
et l'inculcation du genre. À cet effet, le processus de socialisation politique est lui-même sexué (Bargel, 2005). Quelle place occupe cette analyse dans l'interprétation des militant-e$\mathrm{s}$ et militants rencontré-e-s?

En somme, à travers ma recherche, je prévois :

- Vérifier si les militantes et militants perçoivent des rapports de pouvoir genrés au sein de leur collectif.

- Identifier les analyses que formulent les personnes militantes pour expliquer le rapport entre les principes de luttes et les pratiques militantes.

- Situer les interprétations qui réfèrent au genre en tant que structure sociale et celles qui expliquent la (re)production des rapports de pouvoir genrés en terme de dynamique interne.

\subsection{PERTINENCE DE LA RECHERCHE}

Bien qu'un mémoire de maîtrise propose une bien modeste contribution à l'avancement des connaissances, il peut néanmoins comporter un certain intérêt dérivé de sa pertinence sociale et scientifique.

La pertinence de cette recherche consiste notamment à étudier une frange militante peu étudiée, soit la mouvance libertaire. Par ailleurs, l'étude du contexte québécois viendra préciser des analyses principalement ancrées dans une autre culture nationale (surtout française). Je mintéresse à un angle souvent sous-exploité dans les études portant sur la mouvance libertaire, puisque je fais des rapports de pouvoir genrés l'entrée principale. Enfin, comme la littérature s'interroge encore sur les modalités de reproduction des rapports de pouvoir genrés, ma recherche pourra apporter une contribution à ces réflexions.

Dans un premier temps, il s'avère pertinent de s'intéresser à la mouvance libertaire, puisque la plupart des études portant sur le militantisme mixte s'intéressent aux partis politiques, aux syndicats et aux mouvements sociaux, qui se distinguent de la mouvance libertaire sur 
le plan des formes organisationnelles et des principes. Ceci influence nécessairement le régime de genre ${ }^{9}$ des organisations étudiées.

En effet, le milieu libertaire possède des caractéristiques particulières qui me poussent à m'y intéresser pour l'étude des rapports de genre : refus des hiérarchies formelles, désir manifeste et explicite de lutter contre le sexisme, maitrise du langage du féminisme radical, refus discursif de l'assignation des tâches.

Il est évident que le fait de défendre intellectuellement un principe ne se traduit pas automatiquement et nécessairement par l'adoption d'un comportement qui soit cohérent avec les idées défendues. Toutefois, dire cela ne suffit pas à évacuer le questionnement scientifique et sociologique qui sous-tend la recherche d'explications à la (re) production des rapports de genre dans le militantisme, et plus précisément dans l'anarchisme, qui place au centre de sa réflexion et de sa pratique les principes anti-hiérarchiques.

Bien sûr, cette modeste recherche n'apportera pas une réponse définitive aux questions ouvertes et aux spéculations constantes sur les raisons qui sous-tendent la reproduction des rapports de pouvoir genrés dans les collectifs anarchistes. Toutefois, les explications qui concernent les instances militantes en général (partis politiques, syndicats, mouvements sociaux) me semblent insatisfaisantes puisque ces organisations reposent souvent sur des hiérarchies formelles ${ }^{10}$ et ne s'affichent généralement pas ouvertement et publiquement comme féministes. Les interprétations militantes qui y sont présentées font souvent état d'une essentialisation/biologisation des comportements qui est généralement absente chez les libertaires. Les chercheur-e-s rapportent des commentaires ouvertement sexistes et la verbalisation de stéréotypes sociaux sur les genres de la part de leurs interviewé-e-s.

\footnotetext{
${ }^{9}$ Le régime de genre est défini comme étant un « set of arrangements about gender » (Connell, 2002), c'est-àdire la forme particulière que prennent les arrangements de genre dans une organisation: qui s'implique, quelle division du travail, quelles divisions sociales sont créées et reconnues? Bien que correspondant généralement à l'organisation de genre de la société, «The gender regimes of institution usually correspond to the overall gender order, but may depart from it » (Connell, $2002: 54$ ).

${ }^{10}$ Hiérarchies formelles : par exemple, le fait d'avoir une présidence, une vice-présidece et autres postes attribués par un système méritocratique (le vote majoritaire).
} 
En second lieu, je m'intéresse à l'opérationnalisation des rapports de genre au sein de la culture politique libertaire québécoise, alors que la littérature francophone à propos des rapports de genre dans le militantisme s'intéresse surtout au contexte français ${ }^{11}$.

Or, cette distinction n'est pas que «géographique ». Si les rapports de genre comportent plusieurs invariants, dont une stabilité historique et une constance transculturelle qui se nourrit de stéréotypes similaires (Descarries et Mathieu, 2009), la culture politique nationale influence néanmoins la façon dont les rapports de genre sont vécus et interprétés.

Force est de constater que les contextes «nationaux » exercent une influence déterminante sur les organisations anarchistes, ainsi que sur leur rhétorique de justification des structures patriarcales et des comportements sexistes. Le contexte national est le fruit de conflits historiques passés et actuels; en France par exemple, l'accent mis sur l'universalisme fait en sorte que les revendications des féministes sont perçues comme une entorse à ce principe d'unité (Dupuis-Déri, 2009).

Par contre, au Québec, il est considéré légitime que des groupes sociaux occupant des positions inégales puissent être traités différemment, sans que ce ne soit interprété comme une atteinte à l'Universel (idée de la discrimination positive). Considérées comme une «minorité » économique et politique, il est généralement considéré légitime que les femmes revendiquent des conditions différentes pour atteindre l'égalité. Par ailleurs, grâce à la prégnance qu'a eue le mouvement des femmes québécois, il est «plus difficile » pour les hommes libertaires « de critiquer ouvertement les féministes au Québec qu'en France » (Dupuis-Déri, 2009: 196). Ces éléments influencent donc l'arrangement et l'analyse des rapports de genre, d'où la pertinence d'une analyse du contexte québécois.

\footnotetext{
${ }^{11}$ C'est le cas de Alvarez et Parini, 2005; Bargel, 2005, 2007 et 2009; Dunezat, 1998, 1999 2006, 2007, 2008 et 2009; Falquet, 2005; Filleule, 2008; Filleule et Roux, 2009; Kergoat, 1992, 2001 et 2010; Luck, 2008, Monnet, 1998 et 2001; Roux, 2005; Thiers-Vidal, 2001 et 2002.
} 
En ce sens, les analyses du milieu anarchiste français font état de rapports de pouvoirs genrés qui ne semblaient pas s'appliquer au cas à l'étude : par exemple, la condamnation, par les anarchistes français, d'un campement féministe non mixte (Dupuis-Déri, 2009), la misogynie «décomplexée » ou le mépris du féminisme (Monnet, 2001). Or, dans les collectifs libertaires québécois, l'alternance des tours de parole homme-femme va souvent de soi et fait partie du «politiquement correct», de sorte qu'elle ne fait habituellement pas l'objet d'une remise en question. D'autre part, la non-mixité, quoique critiquée informellement, ne fait pas l'objet d'opposition formelle ou organisée (Dupuis-Déri, 2009: 202).

Il ne s'agit pas de nier que la domination masculine opère dans les groupes québécois, mais plutôt de mettre en lumière les différents registres sur lesquels elle se décline. Les groupes anarchistes français comme québécois utilisent des arguments pour s'opposer aux féministes, mais ce sont sur des questions différentes et en ayant recours à des arguments différents (Dupuis-Déri, 2009).

Troisièmement, si plusieurs recherches portant sur la mouvance libertaire abordent la question des rapports de genre, pour la plupart, il ne s'agit pas de leur principal objet. Bien qu'elles adoptent un cadre d'analyse féministe, Drapeau (2007) et Quirion (2008) étudient les rapports de pouvoir en général, tandis que Kruzynski et coll. $(2005,2007)$ s'occupent surtout de décrire et analyser le milieu antiautoritaire.

Plus près de mon objet, Blais (2008) étudie les rapports entre les féministes radicales et les hommes proféministes réunis en mixité dans une action commune. Thiers-Vidal (2001 et 2002) s'est intéressé au milieu anarchiste, mais sa thèse et son article se concentrent surtout sur l'effet de la position sociale de genre sur la façon de penser les rapports sociaux de sexe, principalement chez les hommes. Pour sa part, Dupuis-Déri a consacré quelques articles (2008 et 2009) au rapport entre le féminisme et l'anarchisme et sur le rapport entre les hommes proféministes et anarchistes et le féminisme. 
Cette recherche, dont l'objet concerne les rapports de genre dans le milieu anarchiste mixte québécois s'avère une recherche relativement originale, qui combine les analyses sur les «logiques patriarcales du militantisme » dans l'ensemble des sphères militantes (partis politiques, syndicats, association de quartier, mouvements sociaux) afin de déterminer comment se manifeste la domination masculine dans le militantisme et comment les pratiques militantes contribuent à la reproduction des rapports sociaux de sexe (Roux et Filleule, 2009), avec les analyses qui portent sur les rapports de pouvoir (de race, de sexe, de classe) dans le milieu libertaire.

En dernier lieu, malgré le fait que les collectifs libertaires constituent une réalité relativement marginale, même au sein de la gauche (Delisle-L'Heureux et Kruzynski, 2007), il n'en reste pas moins que la façon dont les rapports de genre s'y articulent et y sont analysés par les personnes militantes s'avère pertinente pour une compréhension plus large de leur persistance à d'autres échelles. En effet, cette recherche étudie les obstacles les plus subtils à la reproduction des rapports de pouvoir basés sur le genre. Je porte mon attention là où la volonté explicite de lutte au sexisme semble ne pas suffire à l'éradiquer, là où est révélée toute la force du genre en tant que structure sociale (Risman, 2004), mais aussi là où les individus participent à la (re)production des rapports sociaux inégalitaires.

La littérature scientifique discute encore des raisons qui font qu'un désir de lutter contre le sexisme et les oppressions ne se traduit pas par une éradication des rapports de pouvoir genrés. Je souhaite aborder la question sous un angle particulier, soit l'analyse de cette contradiction par les militants et militantes libertaires, espérant y trouver une piste afin de mieux comprendre la reproduction des rapports de pouvoir genrés. Peut-être que porter mon attention sur la façon dont les personnes militantes se représentent et interprètent les rapports de pouvoir dans leur collectif me fournira-t-elle les pistes nécessaires à une meilleure compréhension des facteurs de leur reproduction. Ces rapports de pouvoir sontils visibles? Comment s'écartent-ils des principes défendus? Qu'est-ce qui est fait pour 
assurer une plus grande cohérence entre principes et pratiques?

\section{3 ÉLÉMENTS THÉORIQUES}

Avant d'aller plus loin dans l'analyse, les concepts fondamentaux de cette recherche doivent être identifiés et définis.

Dans un premier temps, je spécifierai ce que j'entends par « rapports de pouvoir » et « rapports de genre », et ce qui les lie. Je montrerai ensuite la centralité de la question de la division sexuelle du travail dans le maintien et la reproduction des rapports de pouvoir genrés.

Pour terminer, je présenterai le concept de «mouvement social sexué », qui permet de mettre en relief la façon dont les rapports de genre sont constitutifs des organisations militantes. Cela me permettra de justifier la pertinence d'une approche qui prend le genre en considération pour l'étude du militantisme et des organisations militantes.

\subsubsection{RAPPORTS DE GENRE ET RAPPORTS DE DOMINATION}

Le concept de «genre » me semble plus pertinent que le concept de « sexe " pour approcher mon objet de recherche. En effet, le sexe renvoie à une réalité biologique fixe alors que le genre désigne le produit d'une construction sociale, qui constitue à la fois un rapport social que je souhaite rendre visible et une catégorie d'analyse. Je montrerai également en quoi le rapport de genre constitue un rapport de domination.

En tant que rapport social, le genre doit être appréhendé en termes de rapport de pouvoir, c'est-à-dire en termes de domination. Le genre n'est pas la simple traduction culturelle des différences naturelles du sexe, comme le proposent certains analystes qui parlent en termes de «culture masculine » et «culture féminine ». Cette conception renvoie aux mêmes univers fixes que le concept de «sexe», et ne s'attarde pas aux mécanismes qui les instituent et les nourrissent. 
En fait, le genre repose à la fois sur un principe de différence et un principe de hiérarchie entre ce qui est perçu comme constituant un marqueur de différence (le corps). Ce double principe implique un rapport de domination entre groupes inégalement situés: "Ces rapports sociaux reposent d'abord et avant tout sur un rapport hiérarchique entre les sexes; il s'agit bien d'un rapport de pouvoir, d'un rapport de «classe» - et non d'un simple principe de «classement » (Kergoat 2010a :63). Par ailleurs, le genre « constitue plutôt le principe même de la reproduction d'un ordre social inégalitaire caractérisé par la domination du masculin sur le féminin qui surdétermine la différence biologique » (Bargel et Latté, 2007: 63).

En somme, le genre désigne un rapport social de pouvoir qui instaure et crée des catégories binaires : les « hommes » et les « femmes », et les hiérarchisent (Héritier, 2002, Mathieu, 2001, Roventa-Frusumani, 2009). Ces catégories ne constituent pas, par conséquent, des entités naturelles, biologiques ou anatomiques (Héritier, 2002; Monnet, 1998).

Autrement dit, le genre est conçu comme une organisation sociale qui divise l'humanité en deux groupes de sexe, leur assigne des fonctions différentes et hiérarchisées, institue l'hétérosexualité en norme dominante et légitime la domination masculine (Filleule, 2007: 7).

En tant que catégorie d'analyse, la variable « sexe " occulte les variables sociologiques que je viens de décrire, celles qui opèrent entre le sexe biologique et les situations sociales (Alvarez, 2005: 112-114). Pour cette raison, le recours au concept de «genre » permet de mieux prendre en compte le caractère socialement construit des catégories hommes/femmes (Filleule, 2008; Monnet, 1998; Éphésia, 1995) et la primauté des mécanismes sociaux qui assignent les individus à certains comportements et certaines tâches.

Dans une recherche à propos du militantisme, on peut comprendre le genre «à la fois 
comme un outil conceptuel propre à dévoiler certains mécanismes du pouvoir, comme un rapport social de domination qui détermine en partie les pratiques, les mobilisations et les projets politiques » (Roux et Filleule, 2009: 14). Le concept de genre, qui fait référence à la position sociale des individus plutôt qu'à une «essence » masculine et féminine, représente une catégorie d'analyse qui sert mon projet de mémoire.

Il s'agit d'un concept pertinent à l'analyse du militantisme; il inclut l'ensemble des discours et des pratiques qui contribuent à construire la différence sexuelle, constitue en effet un analyseur puissant des rapports de domination du « masculin » sur le « féminin » dans toutes les dimensions des activités sociales (Politix, 2007: 3).

Je voudrais réitérer le fait que je considère les rapports de genre comme des rapports de domination. Comme je l'ai dit plus tôt, les genres masculin et féminin sont définis et hiérarchisés via un rapport social. Ce rapport social n'est pas neutre; il est inégalitaire, basé sur la domination du groupe des hommes sur celui des femmes ${ }^{12}$. Ce double processus (différenciation, hiérarchisation) s'observe également au sein des pratiques militantes libertaires. En effet, le genre est un marqueur de la position sociale et un type de rapport social qui assigne des individus à une certaine place dans les rapports sociaux et dans la division du travail. La prise en compte de ce rapport de pouvoir permet de parler en termes de domination masculine et non seulement en termes de différence culturelle: «Penser le genre en termes de différence plutôt que de domination nie le procédé par lequel les différences sont créées et le pouvoir attribué » (Monnet, 1998 : 22).

Ainsi, les rapports sociaux de sexe sont définis comme la forme de rapports de pouvoir qui produisent une des divisions fondamentales de la société : celle entre groupes sociaux de sexe antagoniques. La division sexuelle du travail constitue l'enjeu autour duquel ces groupes sont sans cesse recréés, reconfigurés (Kergoat et coll., 1992 citée par Bargel et

\footnotetext{
${ }^{12}$ Delphy, 2001; Bargel, 2005; Vidal, 2004; Ephesia, 1995; Daune-Richard et Devreux, 1992; Delphy, 2001; Devreux, 2004; Drapeau, 2007; French, 1984; Guillaumin, 1978; Mathieu, 1991)
} 
Dunezat, 2009). La centralité de la division sexuelle du travail dans la reproduction des rapports de genre sera abordée un peu plus loin.

Les rapports sociaux de sexe ne sont pas des relations aléatoires : ils font système et sont supportés par un système qui organise ces rapports particuliers, à travers des institutions politiques, juridiques, médiatiques, culturelles. Ce système, le Patriarcat, permet qu'un groupe/classe de sexe se trouve en position de pouvoir ET qu'il contribue au positionnement social (l'exclusion structurelle des femmes) des genres par ces rapports de pouvoir. Le Patriarcat est donc le système social qui permet l'oppression d'un groupe social d'opprimées, les femmes (Delphy, 2001). Il précède de loin l'avènement du capitalisme mais se retrouve renforcé par lui, et est profondément enraciné: grâce à lui, les inégalités sont institutionnalisées, transformées en simples «différences», qui sont socialement légitimées (Ribé, 2009: 164).

En somme, lorsqu'on parle de rapports de genre, il se rappeler qu'il est question d'un rapport social de domination. Le genre peut être assimilé à une tension qui traverse la société, et qui se cristallise autour d'enjeux, pour lesquels s'affrontent les groupes sociaux en confrontation (Kergoat, 2010b: 28). Dans le cas des rapports sociaux de sexe, la tension se fait sentir autour de l'enjeu central de la division du travail. La tension désigne surtout les intérêts divergents des groupes sociaux et non seulement le conflit ouvert.

\subsubsection{POSITIONS SOCIALES ET POSITIONNALITÉS}

Les rapports de pouvoir (basés sur la race, la classe, le sexe) ont tous comme substrat la conjonction d'un double mécanisme de différenciation et de hiérarchisation des différences (Anthias, 1998), comme je viens de le montrer pour la question du genre.

En fait, la réflexion d'Anthias (1998) permet de comprendre que le genre réfère à la fois à une position dans les rapports sociaux (un résultat) et à une positionalité, qui représente le mécanisme d'assignation à ces positions: 
"La notion de «positionnalité " comprend à la fois une référence à la position sociale (comme un ensemble d'effets; soit comme un résultat) et au positionnement social (comme un ensemble de pratiques, d'actions et de significations, soit comme un processus) [...] Ainsi, la « positionnalité » est l'espace à l'intersection des structures (position sociale/impacts sociaux) et de l'" agencéité » (positionnement social/sens et pratique » (Anthias 2001, citée par Kruzinsky, 2004) ${ }^{13}$.

Cette analyse illustre les mécanismes par lesquels les individus sont socialement situés. Les différentes étiquettes catégorielles attribuées aux individus (sexe, classe, race) révèlent des positions dans l'ordre social en vertu des catégories attribuées. Anthias montre comment les options et les possibilités sont semblables chez des individus similairement situés (Anthias, 1998), Cette position détermine les potentialités pour l'individu et pour la catégorie sociale auquel il/elle est rattaché-e. En effet, les étiquettes qui nous sont attribuées (noire, femme, lesbienne) ne font pas que dévoiler une identité; elles révèlent une position dans l'ordre social, et déterminent les perspectives de vie des individus (Anthias, 1998: 507), et, dans le même ordre d'idée, leurs possibilités militantes.

En effet, certains « statuts externes » prédisposent à prendre le côté avantageux de la relation de pouvoir (être un homme, être blanc, être hétérosexuel, être scolarisé...) (Landry, $2007: 343)$

Par conséquent, les rapports de pouvoir font intervenir des forces qui dépassent, sans l'exclure, le niveau interindividuel pour révéler des structures sociales à l'œuvre. C'est pourquoi Anthias rajoute la notion de positionnalité, qui réfère aux processus de stratification/hiérarchisation des catégories, aux processus de reproduction des conséquences sociales inégales et aux processus par lesquels elles sont incarnées dans des institutions, structures, lois, relations de pouvoir. Les positionnalités, en tant que mécanismes, font appel au domaine de l'expérience (relations où chacun-e est défini-e comme différent-e et assigné-e à une place), de l'organisation (comme les catégories imprègnent l'appareil organisationnel) et de la représentation symbolique des catégories de

\footnotetext{
${ }^{13}$ C'est moi qui souligne en gras.
} 
différence (Anthias, 1998). «Ainsi, la « positionnalité » est l'espace à l'intersection des structures (position sociale/impacts sociaux) et de l'«agencéité) (positionnement social/sens et pratique) » (Anthias 2001, citée par Kruzynski, $2004: 230-231$ ).

\subsubsection{DES RAPPORTS DE POUVOIR GENRÉS?}

En somme, l'étude des rapports de pouvoir genrés dans le militantisme amène à joindre la micro-politique du pouvoir (dans les groupes) à la macro-politique (structures), et ce, afin de politiser et situer socialement les relations entre personnes qui sont assignées à des catégories sociales hiérarchisées. En ce sens, je conçois que le pouvoir est à la fois relation et substance, comme je viens de le montrer.

Ainsi, un «rapport de pouvoir » doit être compris en termes de «pouvoir sur » une autre personne (French, 1986) et-doit être assimilé à une relation de domination, foncièrement inégalitaire qui s'opère entre personnes inégalement situées dans des systèmes hiérarchiques sociaux. Par ailleurs, il importe de reconnaître que ces rapports inégaux relèvent d'une construction sociale, idéologique et historique de la domination des hommes sur les femmes (Hirata et coll., 2000).

Par conséquent, lorsque je parle de rapports de pouvoir genrés, je fais référence à ces rapports de domination et d'oppression qui impliquent une relation inégalitaire entre un homme (en tant que membre du groupe des hommes) et une femme (en tant que membre du groupe des femmes). Ceci implique des relations systématiques (qui font système au sein du Patriarcat). Ces rapports sociaux sont antérieurs et postérieurs à la rencontre, mais s'opèrent néanmoins à l'échelle des relations interindividuelles. La division sexuelle du travail constitue l'enjeu des rapports entre les groupes sociaux de sexe, qui entretiennent un rapport antagoniste autour de cet enjeu (Kergoat, 2010). 
Plusieurs ${ }^{14}$ utilisent une conception démesurément large du concept de pouvoir, qu'ils et elles confondent avec « influence ». Cette confusion occasionne une assimilation de toute relation humaine à une relation de pouvoir (au sens de domination). Or, l'influence implique la liberté du sujet d'adhérer ou non, et implique l'action de convaincre, tandis que le pouvoir concerne la capacité de commander, de s'imposer et de déterminer l'agir des autres. Foucault et d'autres tenants de l'approche relationnelle tendent à penser que toutes les relations humaines sont imprégnées de pouvoir (Piotte, 2007). «L'usage fréquent, mais abusif, de l'expression «relations de pouvoir» en lieu et place de «relation de domination » conduit à réduire la domination à un rapport de force susceptible d'être inversé dans certaines circonstances et à méconnaître les effets irréductibles qui lui sont propres » (Hirata et coll., 2000: 45).

Ce qui caractérise un rapport de pouvoir au sens où je l'entends, c'est le conflit d'intérêts (ce que Kergoat appelle «enjeu ») manifeste ou latent (Lukes, 1974). Ce conflit d'intérêts n'est pas simplement interpersonnel, puisqu'il réfère à différents groupes sociaux et aux institutions qui permettent la permanence des différentiels de pouvoir entre groupes (Lukes, 1974). Les groupes en position de domination ont la possibilité de déterminer les valeurs et les normes sociales, se portant garants de l'universel et désignant les «autres» revendications comme "particulières » (Hirata et coll., 2000). L'une des caractéristiques d'un rapport de pouvoir (domination) est que le groupe en position de domination déploie des efforts pour empêcher les demandes «particulières » et leur mécontentement de se traduire en demandes politiques (contrôle de la mise à l'agenda, empêchement de considérer ces demandes comme universelles) (Hirata et coll., 2000; Lukes, 1974).

Enfin, la confusion entre pouvoir et domination contribue à réifier et dépolitiser les relations de pouvoir, en les confinant aux relations inter-individuelles. Par conséquent, la notion de rapport de pouvoir (domination) dépasse la notion de différend interindividuel

\footnotetext{
${ }^{14}$ Ce sont pour la plupart des auteurs qui s'intéressent à la dynamique de groupe et qui considèrent que le pouvoir et la hiérarchie sont normaux et souhaitables pour le fonctionnement des groupes. Voir par exemple Landry (2007) ou Vercauteren (2007).
} 
(«ce qui apparaît entre individus au fil des rencontre ») (Kergoat, 2010), et revêt une dimension structurelle qui se répercute jusque dans les structures militantes:

Les positions de pouvoir des hommes, au sommet des institutions sociales, depuis des siècles, leur ont permis de structurer les institutions, de créer des lois, de légitimer des contenus de connaissance, d'établir des codes moraux, en imposant leur style de gouvernance, les normes et les comportements masculins dans la directions des institutions sociales et de la famille (Roventa-Frusumani, 2009:63).

\subsubsection{LA CENTRALITÉ DE LA DIVISION SEXUELLE DU TRAVAIL}

Comme mentionné au point précédent les rapports de pouvoir se nouent autour de la question de conflit d'intérêts et d'enjeux fondamentaux : "Le rapport social peut être assimilé à une tension qui traverse la société; cette tension se cristallise peu à peu en enjeux autour desquels, pour produire la société, pour la reproduire ou pour «inventer de nouvelles façons de penser et d'agir », les êtres humains sont en confrontation permanente. Ces enjeux sont constitutifs des groupes sociaux " (Kergoat, 200I: 87). Les rapports sociaux de sexe sont organisés autour du concept de la division sexuelle du travail (Kergoat, 2001). Cette division du travail est consolidée par les relations de pouvoir et par le système du pouvoir (le Patriarcat) (Roventa-Frusumani, 2009), ce qui en fait le «nœud de l'oppression des femmes » (Bereni et coll., 2008).

Je définirai ici le concept de division sexuelle du travail, puis j'en montrerai la centralité comme enjeu fondamental des rapports de genre (Juteau, Kergoat, 1992 et 2001; Bereni et coll., 2008). Enfin, j'aborderai la question plus précise de division sexuelle du travail militant.

En fait, la notion de « travail » doit être prise dans son acception large, c'est-à-dire comme "production du vivre », au-delà de l'idée du travail salarié ou professionnel, (Kergoat 2001). En incluant le travail domestique et invisible, la «relation de service » qui caractérise souvent la relation des hommes et des femmes est mise en lumière. Dans le cadre militant, l'acception large de la notion de « travail » permet de prendre en compte des 
tâches comme les efforts de gestion des rapports de pouvoir ou bien le soutien émotif, l'entretien de la dynamique de groupe.

Le concept de division sexuelle du travail (DST) désigne une assignation des activités sociales (travail) entre les groupes sociaux des hommes et des femmes, à travers leur rapport mutuel, qui se déclinent en termes de domination. La DST se décompose en deux mécanismes : la ségrégation horizontale (principe de séparation) et la ségrégation verticale (principe de hiérarchie) (Hirata et coll., 2000).

La ségrégation horizontale désigne le fait qu'il y a des tâches « de femmes » et des tâches «d'hommes ». La DST opère en divisant le "professionnel », «public» (homme) et « domestique » « privé » (femme) et légitime les rapports asymétriques entre les hommes et les femmes (Descarries et Mathieu, 2009). Or, ces sphères sont en fait articulées l'une avec l'autre; c'est le fait du travail gratuit des femmes qui détermine ce qui est domestique et non la nature des tâches effectuées (ces tâches varient selon les contextes culturels et historiques). Par ailleurs, c'est le travail gratuit des femmes qui permet à la sphère marchande, et à l'économie capitaliste de fonctionner (Bereni et coll., 2008; Delphy, 2001). «Le système capitaliste se développe par l'activité productive des travailleurs (hommes) et la reproduction non payée assurée par la femme du prolétaire (la «prolétaire du prolétaire ») » (Roventa-Frusumani, 2009: 16).

La ségrégation verticale réfère au principe de hiérarchie qui fait en sorte que les tâches dévolues aux femmes sont dévalorisées par rapport à celles des hommes, soit sur le plan symbolique (faible reconnaissance, déni du statut de travail), soit sur le plan salarial (faible rémunération). Par conséquent, les tâches des femmes sont rarement considérées comme du «travail », puisqu'elles sont analysées par la prolongation des qualités naturelles des femmes et non comme émanant d'une compétence quelconque (Filleule, 2008; Hirata et coll., 2000). 
La DST a, à travers le temps, contribué à la construction de stéréotypes et attentes de genre, ce qui fait que le commandement, l'autorité, l'autonomie, la création et l'originalité sont généralement associés à la masculinité alors que le service, le soin, l'assistance, le soutien psychologique, le statut subordonné sont associés à la féminité (Descarries et Mathieu, 2009).

Bien que cela constitue une étape importante dans la réflexion sur les rapports de genre, s'arrêter aux "positions », c'est-à-dire au constat de la différence entre les tâches assignées aux femmes et aux hommes, est insuffisant. Les «positionnalités» doivent aussi être prises en compte. Ainsi, il est crucial de s'intéresser aux mécanismes par lesquels le travail est assigné. La division sexuelle du travail repose sur un type particulier de rapports de pouvoir; le rapport de genre (ou rapport social de sexe) (Bereni et coll., 2008).

La division du travail entre les hommes et les femmes représentent, dans la perspective féministe matérialiste et radicale, l'un des mécanismes les plus prégnants de l'oppression des femmes. Il s'agit de la base matérielle et économique du système de subordination des femmes (Delphy, 2001, Hirata et coll., 2000) et s'incarne dans le mode de production domestique, longtemps déconsidéré puisque ce travail n'apparait pas lié au marché et à la sphère de la production marchande; il se caractérise plutôt par le don, où la gratuité du travail des femmes est prise pour acquis (Delphy, 2001).

Par conséquent, la tension fondamentale, l'enjeu, ou le conflit d'intérêts qui caractérise les rapports de genre en tant que rapports sociaux est la question du travail. Plus précisément, on fait appel à la question de la division du travail; qui fait quoi, et quelle est la valeur relative des tâches effectuées par chaque «sexe». En somme, les groupes sociaux sexués se construisent par tension, opposition et antagonisme, autour d'un enjeu : celui de la division du travail (Kergoat, 2001a).

Dans la sphère militante, la division sexuelle du travail est transversale, en ce sens que les 
processus de séparation et de hiérarchisation se retrouvent dans plusieurs milieux et époques. Ainsi, au sein du militantisme, la division sexuelle opère à droite, comme à gauche, jusque dans les organisations dites «progressistes » ou «féministes » (Bargel et Dunezat, 2009; Roux et coll., 2009).

Recourir au genre comme catégorie d'analyse permet de rendre compte de la DSTM et de rendre visible à la fois la ségrégation horizontale et verticale, tout comme la tendance à la dévalorisation (ou carrément invisibilisation) du travail des femmes. De plus, ce concept permet de mettre en lumière le caractère sexué des organisations militantes mixtes.

En effet, les militantes sont souvent assignées à du travail invisible, qui n'est reconnu par aucune rétribution sociale ou symbolique, puisqu'il est perçu comme un prolongement de leurs dispositions naturelles, et se solde par l'appropriation collective de leur travail (Filleule, 2008, Kergoat, 2001). Les principes de séparation et de hiérarchie opèrent à travers la captation, par les hommes, des tâches à forte "valeur ajoutée ». Cette appropriation semble généralement légitime aux militant-e-s puisque l'idéologie naturaliste enseigne que le support émotif ou le maintien de l'harmonie ne sont pas du travail, mais constitue plutôt une prolongation des qualités naturelles des femmes, leur tendance naturelle au «caring» (Anthias, 1998, Kergoat 2001a, Zabalazza). Or, la mouvance libertaire contemporaine étant articulée autour de l'idée de vivre «ici et maintenant »ses idéaux anti-domination, le travail effectué pour éradiquer les rapports de pouvoir (surtout effectué par les militantes) devrait conséquemment être considéré comme du travail politique.

Or, le travail de gestion des rapports de pouvoir se situe à l'opposé d'une tâche « rétribuable», c'est-à-dire une tâche qui donne lieu à une reconnaissance publique (porteparole média, conférence, animation d'atelier) ou qui contribue à développer des compétences/contacts qui sont transférables à la sphère professionnelle, là où l'engagement politique façonne l'orientation professionnelle (Bargel, 2005). Ces tâches rétribuables sont 
plus souvent effectuées par des hommes. Ainsi, l'on voit comment les principes de séparation et de hiérarchie s'appliquent au militantisme.

\subsubsection{LES MOUVEMENTS SOCIAUX SEXUÉS}

La transversalité des rapports de genre et la centralité de la division sexuelle du travail, jusqu'au cœur du militantisme, justifient le recours à une approche en termes de mouvement social sexué.

Le concept de mouvement social sexué (MSS) est introduit par Kergoat (1992) et repris par Dunezat (1997, 1998, 2006, 2007 et 2008) et Bargel (2005). Ces auteur-e-s s'efforcent de théoriser l'action des rapports de genre sur la constitution et le développement des mouvements sociaux. Cette conception a été rendue possible grâce au développement de la sociologie des mouvements sociaux, qui les a longtemps considérés comme étant «neutres», c'est-à-dire que les pratiques masculines (blanches, de classe moyenne) définissaient une norme à laquelle les «autres» (femmes, pauvres, noir-e-s) devaient s'adapter en intégrant les comportements des groupes dominants.

Je présenterai les développements de la sociologie des mouvements sociaux qui ont rendu possible la prise en compte du «sexe » et du «genre » dans les mobilisations mixtes, puis je définirai le concept de mouvement social sexué. Je terminerai en justifiant la pertinence du recours à ce concept pour ma recherche.

Dans un premier temps, la sociologie des mouvements sociaux a évolué vers une prise en compte graduelle du sexe, du genre et des rapports de genre. Jusqu'à la fin des années soixante-dix, la sociologie des mouvements sociaux a été une histoire conceptuelle largement asexuée (Bargel et Dunezat, 2009; Hirata et coll, 2000). En se voulant «neutre», c'est-à-dire indifférente aux rapports de classe, de « race » ou de « sexe » qui structurent le militantisme, la sociologie a invisibilisé tant les «luttes de femmes » que la contribution des femmes (Trat,1997; Hirata et coll., 2000). Ce sont «les angles morts des analyses 
traditionnelles », qui ignorent l'effet structurant des rapports de genre sur la dynamique interne du mouvement, la formulation des revendications et l'évolution de ce mouvement (Filleule, Mathieu et Roux, 2007).

La sociologie des mouvements sociaux a évolué et, graduellement, s'est intéressée au rapport entre le sexe et le militantisme, et ses effets sur la dynamique des mouvements sociaux. Au départ, elle s'intéresse à la «place des femmes » dans les mouvements sociaux. Le genre y alors est utilisé comme équivalent du sexe. Cette approche, basée sur le «gender gap » demeure utilisée aujourd'hui; ces auteur-e-s traitent encore les pratiques masculines comme la norme, ce qui veut dire que les pratiques militantes n'y sont pas vues comme « genrées » ou « sexuées » (Bargel et Dunezat, 2009).

C'est vers la fin des années soixante-dix que la sociologie des mouvements sociaux commence à s'intéresser aux rapports sociaux de sexe (RSS), ce qui permet l'analyse des rapports de domination "qui constituent et structurent les mobilisations et le militantisme » (Bargel et Dunezat, 2009). Cette nouvelle approche permet aussi de penser la sexuation du social, c'est-à-dire la façon dont les RSS imprègnent le tissu social. Au sein du militantisme, cela signifie que ce sont, en partie, les rapports entre les groupes de sexes qui contribuent à la (re)production du genre dans les mobilisations mixtes. L'avantage de l'approche en termes de MSS est qu'elle prend en compte l'existence de la domination masculine au sein même des mobilisations, ce qui fournit une analyse plus riche que les approches $\mathrm{du}$ 《gender gap $»^{15}$ ou l'approche des contraintes externes (Militantisme et hiérarchie, 2007).

Using gender as a conceptual hinge for theorizing social movements has exposed eurocentric, middle-class male biases lurking in frameworks and perspectives assumed to be universal, or at least universally applied (Kaplan, 1009; Ferree, 1992; Noonan 1995; Robnett 1996, 1997). The application and critique of social movement theory through a gender lens

\footnotetext{
${ }^{15}$ L'approche du « gender gap » parle en termes de différences d'attitude entre les hommes et les femmes. Si cette approche identifie des inégalités, elle ne remet pas en question la construction de ces différences, ce qui fait qu'elle risque de verser dans l'essentialisme de sexe et d'analyser le « gap " comme un fossé à traverser pour les femmes, les pratiques masculines étant considérées comme la norme.
} 
has expanded and transformed our knowledge about social movements and gender both generally, and as expressed in particular national, class and cultural context and historical junctures (Kuumba, 1992).

C'est Danièle Kergoat (1992) qui a introduit le concept de « mouvement social sexué » dans son étude sur une mobilisation mixte, dans laquelle, en dépit de leur nombre majoritaire, les femmes ont dû lutter pour se constituer en «sujet collectif» afin de réussir à garder la direction du mouvement de la grève des infirmières. C'est ce qui a amené Kergoat à conclure que l'analyse des mobilisations doit impérativement prendre en compte les rapports sociaux de sexe afin de voir comment ceux-ci « concourent à formuler les objectifs de lutte, objectifs qui peuvent devenir alors des enjeux collectifs pour les hommes et pour les femmes » (Kergoat, $1992:$ 122). C'est ainsi que l'analyse des mouvements sociaux s'enrichit; on ne considère plus les femmes comme un «plus » qui vient "colorer» le militantisme; on reconnaît que les rapports sociaux de sexe sont constitutifs des organisations militantes et en structurent les développements subséquents.

En somme, il s'agit de considérer que les organisations militantes sont façonnées par les rapports de genre. Les mouvements sociaux et les organisations ne sont pas «neutres »; ils font intervenir des groupes sociaux qui y partagent les mêmes tensions et antagonismes que dans la société en général (Dunezat, 1997). Ainsi, « la démarche, en termes de mouvement social sexué a orienté différents travaux montrant que les rapports sociaux de sexe - avec d'autres rapports de pouvoir (classe, race., etc.) - jouent sur toute la dynamique d'un mouvement social et sur les trajectoires militantes, processus qui contribuent à fabriquer la sexuation du social » (Dunezat, 2007: 269) genèse, sélection des revendications et des stratégies, trajectoire du mouvement.

Malgré que le genre fasse l'objet d'un intérêt croissant dans les sciences sociales françaises, particulièrement en sociologie des mobilisations et des «études sur les femmes», la sociologie dominante (certain-e-s diront: traditionnelle) ne prend pas nécessairement en compte les RSS dans l'analyse des mobilisations et du militantisme mixte, ou utilise le 
genre comme équivalent du sexe. Le concept de MSS a été très peu repris dans la sociologie des mobilisations (Trat, 1997) même si plusieurs chercheuses féministes prennent les RSS en compte dans leur analyse, sans nommer le concept comme tel.

Le concept de «mouvement social sexué » (MSS) offre des avantages analytiques certains pour mon objet de recherche. Il autorise une conception du militantisme comme un produit ET un mécanisme de (re)production des rapports de genre (Bargel et Dunezat, 2009). Le genre y est par conséquent approché comme «un système politique qui organise les rapports de domination et comme un processus que les militant-e-s mettent en œuvre dans leurs pratiques, leurs mobilisations et leurs projets politiques » (Roux et coll., 2005 : 9).

En même temps, le concept des MSS permet de rendre visible la division sexuelle du travail militant, en mettant en lumière le travail invisible (sans rétribution sociale ou symbolique) effectué par les femmes militantes (Filleule, 2008).

Aborder mon projet de recherche sous l'angle théorique des rapports sociaux de sexe me permet de prendre en compte le niveau de la dynamique interne des collectifs de l'UCL comme lieu de reproduction des rapports sociaux. L'approche en termes de MSS offre plusieurs possibilités analytiques. D'abord, elle permet de s'interroger sur plusieurs dimensions du mouvement social (entre la dynamique interne et les structures sociales) en axant la réflexion sur les mécanismes par lesquels les femmes sont exclues et de comprendre que les modes d'organisation sont genrés (Filleule, 2008). D'une part, il s'agit de s'intéresser aux « raisons externes », c'est-à-dire la division et la hiérarchie des sexes comme principes organisateurs de la société, en tant que structures sociales. Il s'agit aussi de responsabiliser les individu-e-s comme vecteurs de reproduction, mais de résistance aux structures sociales. Il s'agit de s'interroger sur la façon dont le fonctionnement de l'UCL est genré et correspond à une structuration androcentrée. Ainsi, l'approche en terme de MSS me semble pouvoir faire le pont entre les approches structuraliste et de l'agencéité, les deux niveaux étant évidemment interreliés. 
Par ailleurs, l'approche en terme de MSS permet, en étudiant les rapports entre le groupe de sexe dominant et le groupe de sexe dominé, d'expliquer: la sous-représentation des femmes, les comportements en réunion, la division du travail, les structures, règles, modes d'actions, la sélection des revendications. Dunezat fait l'hypothèse que les rapports de genre au sein de l'organisation en façonnent la structure et les règles de fonctionnement (Dunezat, 1998). Ce concept permet d'appréhender de façon pertinente les logiques patriarcales du militantisme en mettant à l'avant-plan la sexuation des pratiques militantes, leur nonneutralité de genre qui en colore tous les aspects (Militantisme et hiérarchie, 2007).

En somme, les genres «masculin » et «féminin » sont considérés comme des entités construites par un rapport social. Ce rapport social n'est pas neutre; il s'agit d'un rapport de pouvoir articulé autour d'une tension fondamentale; l'enjeu de la division sexuelle du travail. Ce mécanisme, procédant d'un rapport de domination des hommes sur les femmes, assigne ces dernières à des positions subalternes dans l'ordre social. Par contre, même si les rapports sociaux comportent une dimension conflictuelle, ils ne se caractérisent pas par un conflit ouvert prenant l'aspect d'un face-à-face entre deux groupes : les hommes ne luttent pas, «ils ne font que remoduler incessamment au mieux de leurs intérêts à travers leur enjeu, la division sexuelle du travail » (Kergoat, 2010a : 65). Appliquée au militantisme, cette approche permet de réfléchir les organisations militantes et les mouvements sociaux à travers le prisme du mouvement social sexué. 


\section{CHAPITRE 2}

\section{CADRE MÉTHODOLOGIQUE}

Afin de répondre à ma question de recherche de façon cohérente et scientifique, j'ai entrepris une recherche qui repose sur l'étude de cas comme stratégie de recherche, dans une perspective féministe. Ainsi, j'ai étudié deux des trois collectifs locaux qui composent l'Union communiste libertaire (UCL). Les données ont été recueillies par des entrevues semi-dirigées auprès de six femmes et sept hommes, qui sont membres ou sympathisant-es. Le matériel produit par l'UCL (journal Cause Commune, site Internet) a complété la collecte de données, dans une perspective de triangulation.

Ce mémoire s'inscrit dans une approche féministe, c'est-à-dire qu'il adopte une perspective qui vise à rendre visibles les rapports de genre, qui sont généralement occultés dans la recherche traditionnelle (non féministe). Cela implique de mettre en lumière non seulement la sexuation du collectif étudié (Dunezat, 1997), mais aussi les processus qui insèrent les individus dans ces rôles différenciés, en partant des analyses des militant-e-s sur ce sujet

Sur le plan méthodologique, le recours à une approche féministe n'impose pas de méthode de recherche spécifique. Elle implique plutôt un regard, une perspective particulière sur la méthode (Reinharz, $1992: 241$; Ollivier et Tremblay, $2000: 21$ ) et une prise de conscience, toutes deux en lien avec la reconnaissance de la différence genrée et son impact sur le processus de recherche (Fontana et Frey, 2005 : 710-711). D'ailleurs, dans mes entrevues, le genre des interviewé-e-s a influencé non seulement l'analyse qu'ils et elles proposaient, mais également les circonstances d'entrevue (qualité de l'interaction, désirabilité sociale, 
solidarité/antagonisme, séduction, méfiance).

Dans un premier temps sera présentée une mise en contexte de la «mouvance libertaire » ou encore «antiautoritaire » (Kruzynski et Delisle-L'Heureux, 2007). Il s'agira en même temps d'une mise en contexte historique et sociologique, qui me permettra par ailleurs d'insister sur la centralité de la lutte aux systèmes d'oppression, tant sur le plan macro que sur le plan du ici et maintenant.

Ensuite, j'aborderai l'approche féministe de la recherche. À cet égard, la standpoint theory ${ }^{16}$ nous éclaire sur ce qu'elle implique en matière de rapport sujet-objet et en matière d'objectivité. Pour terminer, seront abordés les aspects proprement méthodologiques de la recherche : les techniques de collecte de données et le traitement et analyse de celles-ci.

\subsection{APPROCHE FÉMINISTE, ÉPISTÉMOLOGIE, RAPPORT SUJET-OBJET ET STANDPOINT THEORY}

Dans cette recherche, j'adopte une position épistémologique où je propose une analyse des données à travers le filtre de la théorie du «standpoint ». La standpoint theory propose une réflexion sur le lien entre sujet et objet de recherche. Plusieurs générations d'auteures s'y sont intéressées, des féministes marxistes jusqu'aux post-modernes. En ce qui concerne mon cadre d'analyse, il se situe plus près du« point de vue privilégié » (des groupes opprimés sur les mécanismes d'oppression) que de l'approche en termes de relativité des points de vue.

Selon l'approche que je favorise, la connaissance est vue comme étant socialement située (Campbell et Fonow, 2009; Harding 1995 et 2004; Hirata, 1988; Ollivier et Tremblay,

\footnotetext{
${ }^{16}$ L'usage de l'équivalent francophone de standpoint theory, soit «théorie du point de vue » est rejeté par plusieurs féministes qui lui préfèrent le substantif anglophone, parce que le standpoint ne constitue pas simplement un "point de vue de femmes ". Il s'agit plutôt de conditions sociales et matérielles d'un groupe social (les hommes, les femmes, les beiges, les noires) qui structure la pensée. Je suis d'accord avec cette critique et c'est pourquoi le terme anglophone sera utilisé dans cette recherche.
} 
2000). La définition traditionnelle de neutralité et d'objectivité en science y est remise en question à travers le constat que la production de connaissance est une activité politique, liée aux questions de pouvoir. Par conséquent, le standpoint déconstruit le « faux universalisme » du chercheur « universel » et « désengagé » (Harding, 2004) qui ne serait pas situé socialement. Pour cette raison, les critères qui déterminent la connaissance scientifique (objectivité et neutralité) émanent du groupe producteur de connaissances (surtout des chercheurs de sexe masculin, beiges ${ }^{17}$, économiquement privilégiés, hétérosexuels et occidentaux). Par conséquent, le genre, en tant qu'élément de la position sociale, influence la production de savoir, l'approche de la çonnaissance, en raison du « poids épistémologique du vécu »(Thiers-Vidal, 2002). Ces chercheuses prennent en considération la fonction idéologique de la science, liée aux intérêts particuliers qui orientent le regard de la personne chercheuse (Delphy, 2001).

À cet égard, c'est surtout sur le plan épistémologique que la recherche féministe influence la méthodologie, en s'attachant notamment à considérer que les savoirs et les connaissances sont structurés par la position que l'individu occupe au sein des diverses hiérarchies sociales. L'asymétrie de vécu entre hommes et femmes au sein de ces rapports donne lieu à la même asymétrie dans l'analyse des rapports de genre (Harding, 2004, Thiers-Vidal, 2002). Il s'agit de prendre en considération la fonction idéologique de la science, qui demeure liée aux intérêts particuliers qui orientent le regard de la personne chercheuse (Delphy, 2001). Dans ma recherche, cette approche vaut autant pour les analyses produites par la chercheuse, que pour celles proposées par les militant-e-s lorsqu'appelé-e-s à se positionner sur les rapports de genre dans leur organisation.

De plus, ce mémoire propose une révision des notions traditionnelles d'objectivité et de neutralité, et ce, pour deux raisons principales.

D'une part, mon processus de recherche a impliqué la conjugaison d'un engagement

${ }^{17}$ En référence à la peau plutôt beige des « blancs » (Dupuis-Déri, 2009) 
politique envers la transformation des rapports sociaux de sexe avec le désengagement intellectuel scientifique nécessaire à la production de connaissances scientifiques (Reinharz, 1992). Désengagement qui demeure toutefois relatif, en vertu du fait que la science ne constitue pas une activité sociale neutre (Harding, 2004). Par ailleurs, mon engagement féministe préalable, connu de la plupart des interviewé-e-s, a sans doute eu un impact sur les réponses offertes. Cela a pu orienter les réponses des hommes militants dans une optique soit de désirabilité sociale (répondre de façon à bien paraître aux yeux de l'intervieweuse) ou d'antagonisme (être sur la défensive sachant qu'on a une militante féministe devant soi). Par contre, une solidarité dans l'engagement envers la transformation des rapports sociaux de sexe a sans doute fait en sorte que la qualité de l'interaction a été supérieure dans les entrevues avec les femmes militantes.

D'autre part, ma recherche s'effectue auprès d'un groupe militant dont je suis sympathisante. Je dois par contre spécifier que mon adhésion, qui a coïncidé avec le début de mon étude, n'était pas motivée par des considérations scientifiques, mais bien par une adhésion sincère aux principes de l'organisation étudiée.

Si le fait d'étudier l'organisation dans laquelle je m'implique comporte des avantages indéniables (compréhension des enjeux et des dynamiques, facilité de recrutement d'interviewé-e-s), cela interpelle clairement la question du rapport sujet-objet. Si certain-e-s revendiquent la nécessité de l'extériorité du sujet par rapport à l'objet, d'autres approches se revendiquent plutôt d'une subjectivité assumée, comme les chercheuses et chercheurs du Collectif de recherche sur l'autonomie collective (CRAC) de l'Université Concordia.

Enfin, avec ses forces et ses limites, la subjectivité assumée intègre l'engagement politique à la démarche de recherche et ne nie pas l'impact des biais; elle en propose l'annonce aux lecteurs et lectrices qui pourront se servir des autres recherches sur la question pour la construction de connaissance. Ainsi, la production épistémique repose sur le concept de strong objectivity tel que proposé par Harding (1991), qui renvoie la notion d'objectivité à 
l'extérieur des limites d'un seul propos; par la prise en considération d'un ensemble de propos scientifiques. Étant donné que la connaissance est le produit de rapports sociaux et de la défense de divers intérêts (might makes right), la strong objectivity propose de reconnaître le caractère socialement situé de la production de connaissance, par la détection des valeurs et des intérêts qui sont en jeu dans une recherche (Harding, 1991). Ainsi, plusieurs recherches supposément «neutres » étaient ouvertement racistes et sexistes, parce qu'elles reflétaient les convictions des «faiseurs de science ». Une culture valorisant le sexisme et le racisme définirait ces positions comme «objectives » ou « neutres ».

D'ailleurs, la neutralité n'est pas nécessairement souhaitable: on doit sortir de l'idée traditionnelle qui lie neutralité et objectivité, en ce sens que maximiser l'objectivité est aussi vu comme maximisant la neutralité (Harding, 1991).

\subsection{LA GÉNÉRALISATION}

La généralisation se fait par rapport à des propositions théoriques, et non à des populations ou univers: le cas ne représente pas un échantillon à partir duquel on peut généraliser des théories ou des fréquences. L'univers de généralisation des propositions théoriques (Yin, 1994) de cette étude de cas est la mouvance libertaire.

Le cas que j'ai retenu (l'UCL) est représentatif sur le plan théorique (Roy, $2009: 216$ ), ce qui en ferait un «cas typique " (Yin, 1994). En effet, les principes de lutte de l'Union communiste libertaire rejoignent les idéaux anti-oppression et la «culture politique » du milieu libertaire (tel que définie plus tôt), surtout sur le plan des pratiques organisationnelles: horizontalité, rotation des tâches, démocratie directe, consensus. Je m'attends donc à ce que la littérature identifie en matière de rapports de pouvoir genrés se retrouve aussi dans l'UCL.

Par contre, certaines caractéristiques de l'UCL pourraient influencer le déploiement des rapports de pouvoir genrés et participer à la constitution d'un régime de genre spécifique à 
cette organisation. D'une part, je m'attends à des variations par rapport au contexte français, puisque la culture nationale influence les organisations anarchistes ainsi que leurs logiques de justification (Dupuis-Déri, 2010). De plus, l'UCL est un groupe de tradition communiste qui travaille au développement théorique, à la diffusion d'information et aux plates-formes Internet. Les femmes sont généralement nettement sous-représentées dans ce type de groupe et surreprésentées dans des groupes qui sont plus près des tâches traditionnellement associées aux femmes (Kruzynski et coll., 2007).

\subsection{L'ÉTUDE DE CAS COMME STRATÉGIE DE RECHERCHE}

Cette recherche étant exploratoire, il est pertinent de recourir à un design de recherche reposant sur l'étude de cas. Par ailleurs, le choix de cette stratégie de recherche apparait justifié dans un cas comme le mien, où je souhaite aborder les conditions contextuelles qui influencent les phénomènes investigués (Yin, 1994).

L'étude de cas permet d'étudier en profondeur un cas ou un nombre limité de cas, pour aborder des questions qui dépassent ce cas. En fait, le cas est considéré comme un soussystème qui permet de comprendre un système plus large, notamment le contexte sociopolitique et l'organisation et la société "vue comme un tout intact et intégré » (Gagnon, $2005: 14$ ). Une étude de cas qui repose sur un nombre réduit de sites autorise la prise en compte d'un grand nombre de facteurs de causalité, ouvrant la porte à une profondeur d'analyse supérieure (Roy, 2009).

Le design de l'étude de cas fait l'objet de critiques, notamment en ce qui a trait à la validité interne et la valeur de sa généralisation (Yin, 1994). Or, la recherche qualitative ne vise pas la généralisation statistique, mais plutôt la «généralisation analytique », soit l'extension des conclusions issues du cas à un ensemble de propositions théoriques (Miles et Huberman, 2003; Roy, 2009; Yin, 1994) et à un contexte délimité par la théorie. Dans ce cas, les conclusions de mon étude pourraient s'étendre à des collectifs comportant des caractéristiques similaires. 
En ce qui a trait aux critiques concernant la validité, on accuse généralement l'étude de cas d'être subjective et d'introduire des biais à toutes les étapes (Gauthier, 2009). Pour assurer une meilleure rigueur, j'utiliserai des sources multiples de preuves (Yin, 1994) et je soulignerai les valeurs et intérêts qui caractérisent mon projet de production de connaissance et qui amènent à reconsidérer la notion même d'objectivité.

Afin d'assurer une meilleure validité externe (qui a rapport avec le domaine d'application de la généralisation analytique), il est conseillé de recourir à la réplication, c'est-à-dire de mener des̀ études de cas multiples dès que le temps et les ressources le permettent (Yin, 2003). En raisòn des contraintes liées à la faisabilité ${ }^{18}$, cette étude de cas repose sur un cas unique, mais réparti sur deux sites distincts; chaque collectif local étudié est vu comme une « unité pleine et entière » (Miles et Huberman, 2003 : 311). En effet, puisqu'ils présentent deux dynamiques de groupe séparées, mais s'inscrivent dans la même plate-forme militante, les deux collectifs locaux constituent chacun un « site ».

J'ai utilisé une approche dite « orientée-variable », qui secondarise la dynamique propre de chaque site pour se concentrer davantage sur certains thèmes récurrents transversaux (Miles et Huberman, 2003).

\subsection{LE CAS À L'ÉTUDE : L'UNION COMMUNISTE LIBERTAIRE (UCL)}

J'ai choisi de recourir à l'étude de cas. La façon dont la «mouvance libertaire » est définie dans mon cadre théorique me laissait le choix d'un éventail assez large de groupes au Québec. Certains critères ont permis de réduire davantage les groupes potentiels :

- L'égalité femme/homme et la lutte au sexisme doivent être des principes de lutte explicites (affirmée dans des documents d'information)

- Le collectif doit exister (sous cette forme ou sous une autre bannière) depuis au moins un an et être actif (tenir des réunions sur une base régulière et organiser des actions).

- Le collectif doit être mixte, sans être nécessairement paritaire.

\footnotetext{
${ }^{18}$ Le temps requis pour une maîtrise ne laisse que très peu de temps pour la cueillette et le traitement des données. La réplication n'y est pas possible, en raison également des ressources matérielles limitées.
} 
Mon choix s'est porté sur l'Union communiste libertaire, notamment pour des considérations liées à la faisabilité : mon entrée dans ce groupe a été facilitée par le fait que j'étais sympathisante du collectif local de Chicoutimi. Par conséquent, mon intérêt politique pour l'UCL n'est pas que scientifique. J'adhère également aux principes politiques et aux stratégies de l'organisation, et mon engagement a précédé et suivra ce projet de recherche

L'Union des Communistes libertaires (UCL) existe depuis 2008, en remplacement de la section régionale québécoise de la NEFAC (North Eastern Federation of Anarchist Communists). L'Union régionale du Québec formait une organisation dans l'organisation depuis plusieurs années déjà. D'une part, la question de la langue et les défis liées à la traduction représentaient une première barrière. Par ailleurs, la différence en matière de contexte socio-politique entre les États-Unis, l'Ontario et le Québec rendait difficile l'édification de campagnes communes. L'Union régionale du Québec a rompu avec la NEFAC, et en octobre 2008, l'UCL est née lors du camp de refondation.

L'UCL est une fédération de militant-e-s, réuni-e-s au sein de collectifs locaux, qui s'identifient à la tradition communiste dans l'anarchisme, c'est-à-dire qu'elles et ils endossent les principes du communisme, mais refusent la phase transitoire par un État. Les voies d'action prônées sont l'implication des militant-e-s dans les mouvements sociaux, dans une perspective de radicalisation de ces derniers. Par ailleurs, l'UCL se consacre au développement théorique et à la diffusion des idées anarchistes par des actions collectives et voies de communication (blogues, émission de radio «Voix de faits», journal «Cause Commune $»$, publications).

L'UCL est une organisation plate-formiste, c'est-à-dire qu'elle est organisée autour de certains principes fondamentaux, qui sont inspirés de la plate-forme d'Archinov, un texte de 1926. Ces principes sont :

- l'unité théorique et tactique 
- le fédéralisme et démocratie directe

- la responsabilité collective

- le privé est politique (ne se trouve pas dans la plate-forme originale)

En tant que fédération, l'UCL compte aussi un secrétariat fédéral. Il s'agit d'une instance élective dont la constitution change à chaque congrès annuel. Le secrétariat ne possède aucun pouvoir décisionnel et s'assure principalement de coordonner le travail entre les diverses instances. C'est le Collectif local qui demeure l'instance décisionnelle locale de la fédération; elle a le pouvoir de décision sur les activités locales.

Au moment de la rédaction, l'UCL regroupait trois collectifs locaux en activité :

- Québec : Collectif « La Nuit » : (environ) 8 membres, 8 sympathisant-e-s

- Montréal : Collectif «La Commune» : (environ) 15 membres, 20 sympathisant-e-s

- Chicoutimi: Collectif «Emma Goldman»: (environ) 5 membres, 6 sympathisant-e-s

Auparavant, des collectifs ont été actifs à St-Jérôme, Drummondville et à Sherbrooke. Par ailleurs, l'UCL compte aussi des sympathisant-e-s qui ne sont rattaché-e-s à aucun collectif local.

\subsection{TECHNIQUE DE COLLECTE DE DONNÉES : ENTREVUES SEMI-DIRIGÉES}

Afin de recueillir les données nécessaires à l'analyse, des entrevues individuelles semidirigées ont été menées. La thématique de la recherche et ses objectifs justifient le recours à ce type de méthode de collecte de données.

Le choix de l'entrevue comme méthode de collecte de données m'apparaissait tout indiqué puisque je m'intéresse aux représentations et interprétations de l'articulation des principes et des pratiques en matière de rapports de genre. Les informations fournies par les militant-e-s constituent des données complémentaires et uniques à l'étude d'un objet de recherche. Cela enrichit sans contredit les savoirs théoriques (Guignon et Morrissette, 2006; Ollivier et 
Tremblay, 2000), surtout dans le cas d'une recherche exploratoire où les données existantes ne sont pas suffisantes pour recourir à une enquête par questionnaire.

L'avantage de l'entrevue par rapport à d'autres techniques de collecte de données est qu'elle permet de comprendre des situations complexes (Savoie-Zajc, 2009) en s'appuyant sur la façon dont les individus expliquent leurs pratiques et la façon dont ils les vivent, en recourant à leurs propres termes (Ollivier et Tremblay, 2000).

Afin d'identifier les thématiques pertinentes pour la construction du questionnaire, je me suis basée sur la littérature et sur cette réflexion :

Que faut-il «regarder» pour «voir» les rapports sociaux de sexe? À cette question, il est aisé de répondre «tout» au nom de la transversalité de ces rapports, et nous partageons une telle approche. Elle est cependant difficile à mettre en pratique. L'étude du mouvement des chômeurs et des chômeuses de Morlaix nous a conduits à isoler différents «espaces» permettant de voir fonctionner les rapports sociaux de sexe dans un mouvement social: la répartition quantitative des femmes et des hommes dans le mouvement, le comportement en assemblée générale, le type de structure, la définition des objectifs et des revendications, la division des tâches, les modes d'action, le rapport du mouvement avec son «environnement», les pratiques verbales et physiques dans les rapports entre femmes et hommes, la présence ou non d'un «sujet social femmes» (Kergoat 1992: 123). Le découpage est bien sûr purement théorique (Dunezat, $1998: 169){ }^{19}$

Le guide d'entrevue est constitué de 4 sections. La première recueillait des informations générales et consistait en quelques questions portant sur l'âge, l'ancienneté et le statut de la personne militante. En second lieu, j'ai interrogé les personnes militantes par rapport aux principes de l'UCL afin de vérifier si la personne présentait une sensibilité théorique par rapport au féminisme, et si elle analysait que son organisation faisait montre de cette même sensibilité. Par ailleurs, j'ai demandé aux personnes militantes de me dire ce qu'impliquent pour elles d'être libertaire et d'être féminist. La section suivante concernait les pratiques militantes (ratio hommes-femmes, thématiques des actions, répartition des tâches, prise de parole et leadership). En dernier lieu, j'ai interrogé les militant-e-s à propos des rapports

\footnotetext{
${ }^{19}$ Les italiques sont de moi.
} 
entre principes et pratiques, notamment sur les implications pratiques des principes féministes et égalitaires. Le guide d'entrevue est à l'annexe 1 .

Après les entrevues, j'ai consigné les notes concernant la dynamique d'entrevue, les informations marquantes et le protocole d'entrevue. Des questions ont ainsi été retranchées en cours de route, soit parce qu'elles me semblaient répétitives, soit parce qu'elles ne donnaient pas lieu à des réponses utiles. Par exemple, après quelques entrevues, la question sur la définition du sexisme a été retranchée, puisque je ne m'intéresse pas aux définitions théoriques du sexisme, mais à ses manifestations.

Les 13 entrevues semi-dirigées ( 6 avec des femmes, 7 avec des hommes) ont été réalisées en janvier et février 2011, sauf une, qui a été terminée en juin 2011. Les entrevues, dont la durée a varié entre quarante-cinq minutes et une heure trente, ont eu lieu dans un lieu choisi par l'interviewé-e (domicile, lieu public, bureau). J'ai enregistré les entrevues sur un support numérique audio.

Étant donné la nature qualitative de mon étude et de mon analyse, j'ai recouru à un processus d'échantillonnage théorique, non représentatif du point de vue statistique (SavoieZajc, 2005). Par ailleurs, sachant que la saturation théorique constitue un critère subjectif et hypothétique (Patton, 1990) pour fixer la quantité d'entrevues à réaliser, ce sont surtout les contraintes de la recherche qui ont influencé le nombre d'entrevues. L'échéancier serré et les ressources disponibles (surtout financières) pour effectuer des séjours prolongés à Montréal et à Québec m'ont finalement décidé à réaliser treize entrevues. Je souhaitais interroger un nombre égal d'hommes et de femmes, mais j'ai éprouvé des difficultés à recruter des femmes militantes. J'ai d'ailleurs dû assouplir mes critères dans un cas (celui portant sur l'expérience) afin d'atteindre mes objectifs en matière de recrutement, puisqu'il me manquait une interviewée, étant donné que les femmes sont moins nombreuses à l'UCL, et que la plupart des celles-ci sont arrivées relativement récemment à l'UCL.

Les critères pour la sélection étaient les suivants : 
- Statut : membres ou sympathisantes

- Expérience : personnes impliquées dans le collectif depuis au moins six mois.

- Engagement : avoir participé à au moins la moitié des réunions et actions depuis six mois

Composition de l'échantillon

\begin{tabular}{|c|c|c|}
\hline & Hommes & Femmes \\
\hline Québec & 2 & 2 \\
\hline Montréal & 5 & 4 \\
\hline Total & 7 & 6 \\
\hline
\end{tabular}

\subsection{TRAITEMENT ET ANALYSE DES DONNÉES}

J'ai d'abord transcrit intégralement les entrevues. Par la suite, j'ai effectué un codage intuitif et inductif à l'aide du logiciel libre WeftQda. Les extraits ont été classés, sans catégorisation a priori. J'ai dû refaire le codage, parce que mon inexpérience s'est traduite par la création de codes trop détaillés et aléatoires.

Le second codage a été réalisé à l'aide du logiciel Nvivo 2. Cette ancienne version du logiciel est beaucoup plus conviviale que WeftQda, tout en offrant des fonctionnalités suffisantes. Par ailleurs, ma première expérience de codage a fait en sorte que j'ai pu aborder la seconde de façon plus organisée et plus méthodique. Mon matériel m'était plus familier et j'avais eu le temps de réfléchir sur la forme que pourrait prendre mon analyse.

Mon arborescence a été composée des grandes catégories suivantes:

- principes : anti-oppression, féministes, non hiérarchiques, anticapitalistes, etc;

- activités militantes: publications, actions publiques, développement théorique, division des tâches, leadership, prise de parole, processus, intégration des nouvelles personnes, etc.;

- processus transversaux : division sexuelle du travail, sous-représentation, rapports 
égalitaires, rapports de pouvoir, articulation des luttes, etc.;

- stratégies : disempowerment, féminisation, alternance homme-femme, clarification des malaises, etc.;

- analyses de l'articulation entre principes et pratiques (manque de solution, mieux qu'avant, mieux qu'ailleurs, travail à faire, etc.).

Cette arborescence a servi de structure à la rédaction des chapitres d'analyse : un premier qui expose les contradictions entre les principes et les pratiques, un second qui met en lumière les interprétations empiriques de ces tensions et un dernier qui propose un retour théorique sur la possibilité de la cohérence féministe dans une organisation mixte. 


\section{CHAPITRE 3}

\section{L'ARTICULATION DES PRINCIPES ET DES PRATIQUES EN MATIÈRE DE RAPPORTS DE GENRE : «L'AMBIVALENCE NORMATIVE »}

Comme je l'ai souligné au chapitre précédent, les libertaires "contemporain-e-s 》 (postSeattle) sont organisés autour de principes égalitaires et antisexistes, refusant toute forme de hiérarchie et d'oppression. Or, plusieurs auteur-e-s soulignent l'inégalité des statuts entre hommes et femmes, l'invisibilisation du travail des femmes, leur sous-représentation ainsi qu'une division sexuelle du travail militant dans les mouvements sociaux, dont la mouvance libertaire $^{20}$. Cet écart entre les principes et les pratiques militantes dans les organisations libertaires devait être investigué davantage.

Malgré ces présomptions issues de la littérature, il me paraissait scientifiquement impossible de prendre pour acquis que ces dynamiques allaient nécessairement se retrouver à l'UCL. Il était tout aussi impossible d'en présumer l'arrangement particulier, chaque organisation étant structurée selon un régime de genre ${ }^{21}$ particulier. Je devais donc confirmer à la fois l'importance que revêtent ces principes égalitaires et antisexistes pour les personnes militantes rencontrées et les mettre en rapport avec les pratiques (les mécanismes, les processus d'assignation).

\footnotetext{
${ }^{20}$ Voir entre autres: Bargel; 2005 et 2009; Blais; 2008; Drapeau, 2007; Dunezat; 1998, 1999, 2006, 2007, 2008 et 2009; Dupuis-Déri, 2009; Filleule et coll., 2007; Filleule, 2008; Kergoat, 2001; Luck, 2008; Momet, 2001; Quirion, 2008; Roux, 2005 et 2009.

${ }^{21}$ Le concept de régime de genre désigne les formes et arrangements particuliers des rapports de genre dans une organisation (qui est recruté, comment est divisé le travail, quelles divisions sociales sont reconnues). Les régimes de genres correspondent souvent à l'ordre de genre de la société, mais peuvent aussi s'en distinguer (Connell, 2002)
} 
Ce chapitre vise à mettre en lumière "l'ambivalence normative » entre ce qui est valorisé sur le plan du discours et ce qui est révélé par la pratique militante. À travers l'illustration de la tension entre, d'une part, les principes valorisés, et les inégalités (re)produites dans la pratique militante ${ }^{22}$, je cherche à montrer les zones de tension et la « sexuation du social », à travers l'organisation du travail militant, pour voir «comment les rapports sociaux de sexe traversaient les mobilisations » (Dunezat, 2007) ${ }^{23}$.

Je présenterai en premier lieu les analyses que les interviewé-e-s proposent des principes qui caractérisent leur organisation et la mouvance libertaire en plus des motivations individuelles des militant-e-s à s'impliquer dans l'Union communiste libertaire (UCL). Dans un deuxième temps, je mettrai en relief les diverses oppositions entre principes et pratiques.

\subsection{LES PRINCIPES CENTRAUX SONT D'ABORD ANTICAPITALISTES}

Dans l'approche du MSS, il est pertinent de s'interroger sur les motivations individuelles à l'engagement. Cela fournit des indices sur les divisons sociales reconnues et considérées prioritaires. Par conséquent, les militant-e-s ont été questionnés sur les principes qui, selon eux et elles, caractérisaient leur organisation et la mouvance libertaire. Par la suite, je les ai invité-e-s à s'exprimer sur le cheminement et les raisons qui les ont amenés à s'impliquer à l'UCL en particulier. Tant les principes nommés que les motivations individuelles à l'engagement permettent de conclure à la centralité des principes anti-oppression et anticapitaliste, alors que la question des rapports de genre semble pour sa part moins déterminante, sauf pour les femmes militantes.

\footnotetext{
${ }^{22}$ Ce questionnement est issu de Roux, 2005.

${ }^{23}$ Pour le concept de "mouvement social sexué », voir Danièle Kergoat (1992 et 2001) et Xavier Dunezat $(1998,1999,2006$ et 2007$)$ ainsi que mon cadre conceptuel.
} 


\subsubsection{PRINCIPES ASSOCIÉS À LA MOUVANCE LIBERTAIRE ET À L'UCL}

Que signifie le fait d'être libertaire pour les personnes interrogées? Elles insistent toutes sur l'importance des principes d'autonomie, d'opposition aux hiérarchies et oppressions, en plus de souligner la nécessité d'entretenir des rapports égalitaires les un-e-s avec les autres. La question des relations de genre n'est abordée que par deux hommes, alors que plusieurs militants ne spécifient pas l'égalité des hommes et des femmes. Les femmes militantes insistent davantage sur la question du féminisme, et en font une condition de l'anarchisme.

Tsé être libertaire sans être fëministe, c'est pas être libertaire, à mon anis. Josiane

D'autre part, plusieurs insistent sur la nécessité d'inscrire les idéaux dans la pratique ici et maintenant. Cela laisse supposer que l'abolition des hiérarchies entre les hommes et les femmes devrait aussi être transcrite dans la pratique.

On a discuté de ce qu'on appelle l'éthique libertaire. Qui implique une façon de voir la vie et de voir son quotidien, pis une façon de voir qui est congruente. avec notre vision de l'après-révolution. Ca implique, dès maintenant, d'appliquer des principes de base qui sont critiques des rapports sociaux qu'on qu'on produit et reproduit dans notre quotidien.- Émilie

Par contre, invité-e-s à se prononcer sur les buts et les principes de l'UCL, toutes les personnes rencontrées abordent prioritairement l'aspect communiste libertaire de l'organisation, qu'ils et elles définissent comme la proposition d'une société organisée sur les bases économiques du communisme, sans la domination étatique. Cela suppose donc la centralité du capitalisme et de l'État comme systèmes d'oppression fondamentaux.

Ensuite, les hommes et les femmes n'insistent pas sur les mêmes principes. Après le principe communiste libertaire, les hommes abordent principalement les questions de stratégies, de rupture radicale et de révolution, tandis que les femmes axent principalement leurs interventions sur la question de l'opposition au Patriarcat, des principes féministes et de l'antisexisme, tout en insistant sur l'attrait de la forme organisationnelle du «collectif». Cela laisse supposer que les militantes accordent plus d'importance aux principes féministes et qu'elles y pensent plus spontanément, étant donné que cette lutte concerne une 
oppression qu'elles vivent directement et qu'elles ont intérêt à éradiquer.

\subsubsection{LES MOTIVATIONS INDIVIDUELLES À L'ADHÉSION À L'UCL}

Dans la perspective du mouvement social sexué, la motivation individuelle à participer à une organisation représente un indicateur du rapport social constitutif de celle-ci (Dunezat, 1999). J'ai donc demandé aux répondant-e-s de s'exprimer sur les raisons qui motivent leur adhésion à l'UCL afin d'identifier le rapport social prédominant.

Les militant-e-s se disent d'abord attiré-e-s à l'UCL par l'analyse en termes de rapports de classes (critique anticapitaliste) et de rapports politiques (critique antiétatique). Ainsi, dans ce cas, les militant-e-s mettent davantage l'accent sur les rapports de classes que sur la transformation des rapports de genre et l'opposition au Patriarcat.

D'ailleurs, plusieurs reconnaissent que l'UCL est d'abord une organisation axée sur la lutte des classes, qui présente néanmoins des valeurs (pro)féministes.

L'UCL c'est une organisation qui est vraiment phus lutte-de-classiste que féministe. Et ça, c'est ancré dans la tête de beaucoup, beaucoup de gens, que c'est ça avant tout.- Josiane

Toutefois, ceci ne signifie pas que les préoccupations liées au Patriarcat sont absentes. Cela illustre plutôt la tendance des organisations à valoriser un rapport social en particulier, habituellement, le rapport de classe (Blais et Courcy, 2010), ce qui tend à aplatir (sans toutefois les éliminer) les autres rapports sociaux (Kergoat, 2010). C'est principalement pour les femmes militantes que le renversement du Patriarcat et de défense des valeurs féministes sont déterminant dans la décision d'adhésion.

Ce qui m'a attiré à l'UCL, c'est beaucoup l'idée de, la création de contrepouvoir. Pis en même temps, c'est sûr que je suis allée vérifier s'il y avait des valeurs féministes, ça faisait partie des buts et principes, que ce soit anarcha-féministe, ça m'a beaucoup interpellé. Le fait aussi qu'il y avait d'autres féministes que je connaissais qui en faisait partie, je me suis dit que peut-être qu'au sein de l'UCL cette lutte-là aussi était articulée - Ève-Marie 
Les hommes aussi abordent ces questions, mais souvent au sein d'une énumération.

\subsubsection{LES MILITANT-E-S PRÉSENTENT DIVERS DEGRÉS DE FÉMINISME}

$\mathrm{Au}$ niveau individuel, toutes les personnes interrogées s'identifient comme féministes, se situant dans une approche matérialiste et/ou radicale. Certaines reconnaissent s'inspirer des réflexions queer, bien qu'il ne s'agisse pas de l'élément central de leur approche.

Bien que la sensibilité féministe de l'ensemble des militants soit jugée variable, aucun ne remet ouvertement en question la non-mixité, l'analyse en termes de genre ou le bien-fondé du féminisme :

Quand on parle de féminisme, c'est comme tabou dans le mouvement militant, tu peux pas être contre. Ça va paraître dans tes pratiques, mais tu peux pas être théoriquement contre. - Josiane

Plusieurs études portant sur la sphère militante rapportent des analyses biologisantes de la part des interviewé-e-s. (Dunezat, 1998; Bargel, 2007; Quirion, 2008; Quéniart et coll., 2010), qui naturalisent les différences entre les hommes et les femmes pour remettre en question la possibilité de l'égalité : «Selon moi, ça va être difficile d'arriver à un point où on va être égales à tous les points parce que... on peut pas dénier la nature, là » (Quéniart, 2002 : 118). Par contre ces analyses essentialistes sont absentes chez les ucélien-ne-s qui adoptent une vision des genres en tant que groupes sociaux.

Ceci peut paraitre banal, mais cela constitue en fait un effort de déconstruction majeur, les stéréotypes sociaux et la vision essentialiste des sexes étant accessibles grâce à un effort cognitif minimal (Descarries et Mathieu, 2009; Risman, 2004).

Or, plusieurs militant-e-s remarquent que la sensibilité féministe de certains ne se traduit pas nécessairement dans les comportements à l'UCL.

Ce n'est pas tous les membres et sympathisants de l'UCL qui sont féministes 
jusqu'au bout, autrement que dans ce qu'ils se disent. - Yann

Les femmes évaluent que les hommes présentent différents degrés de sensibilité féministe; des hommes proféministes qui agissent en tant qu'alliés, ceux pour qui ce n'est pas une préoccupation (Ceux-ci ne féminisent pas leurs textes ou vont "oublier» de prendre en considération les rapports de genre dans leurs analyses), jusqu'à ceux qui n'ont aucun intérêt pour ces questions.

Mais oui, on voit des personnes dans notre tête. Ces personnes-là, tu te dis wow cette personne-là, elle est tellement... elle est tellement féministe dans ses comportements pis elle est presque égalitaire, pis tandis que tu vois d'autres personnes pis tu te dis : hui, ouf, pas fort. - Josiane

Faque c'est plus des trucs dans les attitudes, c'est ça. Au niveau théorique je pense pas qu'il y ait personne qui appuierait une relation pas égalitaire, mais dans la pratique des attitudes qui se font... qui ont besoin de changer, et moi y compris - Oli

En somme, les thèmes antisexistes, féministes et d'opposition au Patriarcat sont abordées spontanément surtout par les femmes et quelques militants. La prise en compte de ces oppressions est plus déterminante pour les militantes dans leur décision d'adhérer ou pas à une organisation. Par contre, bien que le féminisme et la transformation des rapports de genre ne soient pas considérés comme des axes de lutte centraux, ils demeurent des questions présentes.

Les sections suivantes mettront en relief les points qui traduisent une tension en matière d'articulation des principes féministes et des pratiques qui contribuent à la (re) production des rapports de genre au sein du collectif militant.

\subsection{L'AMBIVALENCE NORMATIVE : L'ÉCART ENTRE LES PRINCIPES ET LES PRATIQUES :}

Bien sûr, le fait d'adopter une éthique égalitariste ne se traduit pas automatiquement par des comportements idoines (Monnet, 2001; Dupuis-Déri, 2008) : «La bonne intention ne suffit donc pas à éliminer la hiérarchie " (Blais, 2008 : 171). En effet, penser que les valeurs d'un 
individu se répercutent nécessairement sur son comportement relève d'une vision idéaliste des individus (Dupuis-Déri, 2008). Selon une militante, les hommes qui adhèrent à l'UCL sont sincères dans leur adhésion aux principes anti-oppression. Plusieurs hommes interviewés soulignent leur propre adhésion aux principes féministes et leur désir d'entrer dans des rapports égalitaires avec les femmes, mais soulignent en même temps que ces principes ne les prémunissent pas contre l'adoption de comportements de domination. Par contre, à cause des principes égalitaires, la reproduction des rapports de genre paraît particulièrement surprenante pour certaines personnes :

Je ne m'attendais pas à ça, les rapports de genre oppressifs quand je suis arrivée comme nowvelle. C'est pas tant que c'était pire qu'ailleurs, c'est que j'avais comme des attentes, un idéal. Je savais que ça allait pas être parfait parce que bon, la réalité et le théorique, c'est deux choses. Mais je ne m'attendais pas à ça. Je pensais que ça allait être mieux, vraiment.- Josiane

Les personnes interviewées identifient des zones où les principes de l'organisation et les pratiques ne coïncident pas. Par contre, au-delà de l'idée de «contradiction », c'est l'idée de la tension qui semble exprimer le mieux la façon dont ce rapport est interprété.

\subsubsection{ORGANISATION PRO-FÉMINISTE ${ }^{24}$ ET SOUS-REPRÉSENTATION DES FEMMES}

Bien que tous les hommes militants n'associent pas spontanément l'UCL aux principes féministes, les buts et principes de l'organisation, eux, en font clairement mention. Par ailleurs, les interviewées considèrent que l'UCL est pro-féministe dans ses principes, mais pas nécessairement dans ses pratiques. Le lien entre la position pro-féministe de l'UCL et la sous-représentation (quantitative et qualitative) des femmes mérite que l'on s'y intéresse. En effet, l'approche du «mouvement social sexué » (Bargel, 2005; Dunezat, 1998; Kergoat, 1992) postule que «l'étude des rapports sociaux de sexe ne peut évidemment pas faire abstraction de cette répartition » (Dunezat, $1998: 171$ ).

\footnotetext{
${ }^{24}$ L'UCL se présente sur son site Internet comme supportant le principe d'anarcha-féminisme en plus de s'opposer, dans ses buts et principes, à la domination des hommes sur les femmes. Cela en fait une organisation pro-féministe, c'est-à-dire une organisation qui appuie la lutte des femmes tout en s'opposant aux oppressions basées sur le genre, sans être nécessairement de sexe féminin.
} 
D'abord, je montrerai que les personnes militantes constatent une sous-représentation des femmes. Ensuite, je mettrai en relief le cadrage féministe (et non libéral) de la notion de parité adoptée par les militant-e-s, puis j'aborderai le rapport de force édifié par les femmes à l'UCL et ses limites. Ce que je souhaite mettre en relief ici, au-delà de l'idée de la tension entre principe et pratique, est le rôle déterminant des rapports de pouvoir dans la sousreprésentation des femmes à l'UCL.

Premièrement, lorsqu'interrogé-e-s sur le ratio hommes-femmes dans leur collectif, les personnes interviewées font toutes le constat de la sous-représentation des femmes. Les évaluations, oscillant entre 20 et $35 \%$ de femmes, correspondent à ce qui est retrouvé dans la littérature (Luck, 2008; Knoll et Aragorn, 2010). Kruzynski et coll. (2007) associent une surreprésentation des hommes aux collectifs dont les activités sont plus «théoriques » (production de journal, développement théorique, informatique), qui sont les activités principales de l'UCL.

Cette sous-représentation est jugée déplorable et problématique par les personnes interviewées. Elles considèrent qu'une organisation avec des valeurs féministes et égalitaires devrait comporter un plus grand nombre de femmes. Un militant souligne que la lutte à la sous-représentation des femmes est nécessaire afin d'être plus cohérent. Ainsi, l'augmentation du nombre de femme témoignerait d'une avancée en matière de rapport de genre. C'est pourquoi les militant-e-s du collectif de Montréal se réjouissent du ratio presque paritaire au sein du nouveau membership, qu'ils et elles analysent comme un résultat du travail qui est fait pour améliorer l'image du collectif et la dynamique interne de l'organisation.

En second lieu, le lien tracé par les militant-e-s entre parité et modification des rapports de genre témoigne qu'elles et ils n'adoptent pas le cadrage libéral, qui conçoit la parité comme un objectif numérique à atteindre, mais le cadrage féministe. En ce sens, elles et ils lient la question du désir d'une meilleure représentation quantitative (nombre) des femmes avec 
celle de la représentation qualitative (la place occupée).

Les femmes militantes expliquent la sous-représentation entre autres en invoquant l'aspect oppressif de la dynamique interne (les rapports de pouvoir genrés). Les militantes qui ont connu la NEFAC soulignent que les rapports de genre y étaient plus oppressifs. « En effet, la proximité des libertaires d'avec la «culture ouvrière » définit la culture militante, ses modes d'action et ses débats, qui se ressent dans son fonctionnement et dans les échanges entre militants » (Luck, 2008 : 586). Ainsi, la centralité des rapports de classe, les modes de communication valorisés et les pratiques témoignent du «caractère institutionnel de la masculinité ». En général, les femmes ne se sentent pas à l'aise dans cette dynamique guerrière (Quéniart, 2002).

Plusieurs interviewé-e-s constatent que depuis la rupture d'avec la NEFAC, les femmes sont plus nombreuses et ont institué un certain rapport de force qui influence les pratiques. Ils et elles établissent un lien entre la présence de féministes et la modification de certains aspects de la domination masculine dans l'UCL, ce qui rend les collectifs plus conviviaux pour les femmes.

Ainsi, elles et ils considèrent que les femmes qui arrivent dans l'organisation ne sont pas qu'un «plus » qui viendrait « colorer » le militantisme (Kergoat, 1992), mais comme ayant un réel impact sur la dynamique interne. Les féministes remettent en question les rapports de genre et viennent donc bouleverser la dynamique organisationnelle et militante (Dunezat, 1998).

Les groupes d'extrême gauche et les groupẹs féministes n'entretiennent pas la même relation avec la question des fins et des moyens de l'action : pour l'extrême gauche (dans la tradition) la fin justifie les moyens. Le mouvement féministe a prêté davantage d'attention aux processus sociaux mis en œuvre dans la lutte contre le sexisme et les oppressions liées au genre (Lamoureux, 2003). C'est d'ailleurs sous l'influence des pratiques horizontales du 
mouvement féministe, en réaction avec les pratiques excluantes des mouvements communistes, socialistes et anarchistes, que les libertaires «contemporains $»^{25}$ ont introduit des formes organisationnelles qui relèvent de la nécessité de rompre avec l'attente du «grand soir »; horizontalité, décentralisation, mode d'organisation réticulaire, qui sont maintenant des pratiques communes dans le milieu anarchiste québécois.

Selon les interviewées, l'augmentation du nombre de femmes féministes dans l'UCL a eu des impacts en matière de dynamique organisationnelle: introduction du point le privé est politique dans la plate-forme organisationnelle, politique de féminisation des textes, introduction de stratégies de gestion des rapports de genre (caucus non mixtes, clarification des malaises) ce qui fait que les militant-e-s de l'UCL observent une amélioration des rapports homme-femmes.

C'était les femmes qui étaient là qui ont réussi à induire un changement du rapport social. Je pense que les femmes reconnaissent plus l'importance du féminisme que les hommes, donc sont plus portées à faire en sorte que nos principes fittent avec nos pratiques. C'est pour ça que c'est plus elles qui développent des stratégies ou qui font en sorte que ça change. Marie-Pier

En matière de modification des comportements individuels en réunion, le rapport de force féministe permet la sanction de certains comportements de domination adoptés par les hommes. Avec le temps, ces comportements se font moins présents;

$Y^{\prime} a$ une certaine sensibilité féministe à l'UCL. Y'a des gens que je ne sais pas si c'est justement parce qu'on leur a rappelé que c'était dans les buts et principes et qu'on a fait justement un travail. D'autres que c'est plus naturel, entre guillemets, que c'est plus intégré, que ça devient de moins en moins qu'ils ont besoin de penser pour l'articuler. Y'a d'autres personnes chez qui c'est plus automatique, qui le font pas parce qu'ils sentent un rapport de force, que sinon ils vont se le faire dire - Ève-Marie

Par ailleurs, selon les militantes, l'augmentation du nombre de femmes a stimulé une solidarité féministe et a instauré un certain rapport de force.

\footnotetext{
${ }^{25}$ Contemporains: désigne les groupes libertaires nés de la mouvance post-Seattle, dans le giron du mouvement altermondialiste. Les libertaires proposent une remise en question plus radicale que ces groupes. Voir Breton, Kruzynski et Sarrasin (2012) et Dupuis-Déri (2010).
} 
Et le fait qu'il y ait de plus en plus de filles, ça fait en sorte que... comment je dirais ça, plus de filles, ça fait qu'une fille qui se sent coupée elle va le dire, alors que si t'es toute seule, tu le dis pas. En tout cas, ça fait comme un effet boule de neige. - Gabrielle

En ce sens, le cadrage féministe de la notion de parité estime que le nombre de femmes joue un rôle dans la possibilité de résister à la domination masculine et de changer la culture militante. Si les hommes sont identifiés comme groupe social en position avantageuse, les femmes doivent, pour leur part, être assez nombreuses pour ne pas se retrouver atomisées (Dunezat, 2007). Ce «brassage » du régime de genre de l'organisation est généralement perçu positivement par les hommes militants rencontrés.

Parce que j'ai l'impression que ça fait comme une roue aussi, on a quelques camarades femmes qui secouent les puces un peu, qui ont beaucoup d'initiative, ben ça invite d'autres femmes, aussi. C'est un peu ça qui s'est passé à Montréal.- Maxime

Par contre, le rapport de force érigé par les féministes demeure. Bien qu'elles soient plus nombreuses qu'autrefois, les femmes demeurent sous-représentées à l'UCL, alors que les hommes restent quantitativement et qualitativement plus présents. Ceci limite une transcription plus littérale des principes féministes dans la pratique. Le processus de transformation du régime de genre génère nécessairement de la résistance de la part des personnes en position privilégiée (Connell, 2002). Ainsi, la construction d'un véritable rapport de force est limité : «Devant ce pouvoir, les femmes ne peuvent opposer qu'une forte hégémonie numérique, à condition que celle-ci s'accompagne d'une interrogation des rapports femmes-hommes traditionnels. $\grave{A}$ cette seule condition, on peut espérer une participation à la fois quantitative et qualitative plus importante des femmes. » (Dunezat, 1998: 179).

Malgré les progrès effectués depuis la refondation, les nouvelles militantes s'insèrent dans un contexte minoritaire structuré par la domination masculine et vivent une intégration parfois difficile (Kanter, 1977, Belle, 1989). Par ailleurs, « the impersonal but pervasive tendency, in organizations dominated by men, to favour criteria and procedures that favour 
men » (Connell, 2002) font que la forme des organisations leur correspond davantage et leur permet de maintenir leur mainmise sur le devenir de l'organisation (sélection des revendications, octroi des rétributions).

Je trouve que l'adaptation pour une femme, c'est vaiment plus difficile, s'adapter à un groupe, parce qu'en phis des relations de pouvoir; majoritairement, y'est composé d'hommes, faque... Moi, je l'ai tellement trouvé difficile, quand je suis arrivée. - Josiane

Mais encore là, si je prends des nouvelles personnes, encore là un nouveau va se sentir phus à l'aise plus rapidement qu'une nouvelle. Ça revient, ça...Yann

Les interviewées soulignent que les rapports de pouvoir peuvent rendre l'intégration difficile aux nouveaux sympathisants, mais surtout aux nouvelles sympathisantes:

Mais au début, si tu connais personne... et c'est vrai que les gars ils parlent plus fort, et qu'ils cèdent pas tout le temps la parole, que y'a des tours de parole, mais ça se coupe, mais c'est pas aussi intégré que dans d'autres milieux. - ל̀ve-Marie

À mon avis, c'est plus difficile pour les filles d'être intégrées dans un collectif. Une nouvelle, à toutes les initiatives qu'elle prend, ce qui arrive c'est : je suis pas d'accord, je te rentre dedans. C'est pas juste, je suis pas d'accord et je formule mon désaccord. C'est comme une coche de plus, tsé y'a pas d'attention particulière sur la façon de formuler les choses.- Émilie

En somme, bien que l'on puisse se demander si une organisation pro-féministe doit ou non être paritaire, et si, dans le cas contraire, la sous-représentation entre en contradiction avec ses idéaux, reste que la répartition quantitative des hommes et des femmes dans une organisation militante signifie quelque chose en matière de rapport de genre.

En effet, la répartition quantitative des hommes et des femmes constitue un indicateur de l'état des rapports de genre à l'intérieur de l'organisation. Une organisation où les pratiques ne relèveraient pas de la domination masculine (c'est-à-dire où les femmes auraient réussi à établir à la fois une hégémonie numérique et un rapport de force dans la remise en question des rapports de genre) permettrait un meilleur investissement des femmes (Dunezat, 1998). La sous-représentation des femmes semble liée, entre autres, aux rapports de pouvoir dans 
l'organisation. En effet, certaines militantes se lassent des pratiques d'invisibilisation et de la négation de l'oppression de sexe et peuvent décider de militer dans une autre organisation (Filleule, Mathieu et Roux, 2007).

\subsubsection{LE PRINCIPE DE ROTATION DES TÂCHES ET LA DIVISION SEXUELLE DU TRAVAIL MILITANT (DSTM)}

Je montrerai ici que selon les militant-e-s, ce sont les principes de rotation des tâches et de volontariat qui gouvernent la division du travail à l'UCL. Ils et elles notent cependant une cristallisation des tâches entre les mains de quelques-un-e-s (surtout quelques-uns) et le maintien de la division sexuelle du travail militant (DSTM). En vertu du rapport entre position et positionnalité, il ne faut pas s'arrêter au constat de la différence entre les tâches assignées aux femmes et aux hommes, bien que cela constitue une étape importante dans la réflexion sur les rapports de genre. Ce qui est crucial, c'est de reconnaître les mécanismes par lesquels le travail est assigné via un rapport de pouvoir : le rapport de genre (Bereni et coll., 2008).

\section{Le principe de rotation des tâches et ses limites}

Lorsqu'interrogées sur la façon dont les tâches sont allouées dans leur collectif d'appartenance, les personnes rencontrées mettent de l'avant la question de la rotation des tâches, principe jugé essentiel:

On essaie de faire rotationner les tâches, mais c'est pas... C'est une préoccupation si on veut. C'est dans les préoccupations des gens, mais pas tout le monde non plus, en fait. - Pierre-Luc

En principe, les tâches sont allouées par volontariat, à main levée ou par auto-proposition. Chaque tâche qui nécessite une prise en charge (animer, rédiger le PV, organiser une activité, contacter un autre groupe, etc.) est offerte à toutes et tous afin d'assurer une rotation, de respecter les capacités, les disponibilités et le degré d'engagement de chacun-e. Les interviewé-e-s soulignent que personne n'est forcé à faire quoi que ce soit. D'autres postes (la trésorerie, les tâches liées au secrétariat fédéral) sont électifs et une rotation est faite une fois par année. 
D'autres méthodes d'allocation des tâches sont expérimentées pour tenter d'assurer une meilleure rotation. Par exemple, pendant quelques mois, les responsables à l'animation et au secrétariat ont désigné-e-s par pige. Lors du tour de table préalable à la pige, les personnes qui ne se sentaient pas d'humeur à animer pouvaient le mentionner. De plus, après avoir constaté une division sexuelle du travail lors des camps de formation et des congrès, l'allocation des tâches ménagères et alimentaires a été modifiée : au début du camp, chaque personne devait s'inscrire dans une équipe responsable d'un repas et du nettoyage.

Ces deux exemples illustrent à la fois l'importance pour les ucélien-ne-s du principe de rotation et le fait que la rotation des tâches n'est pas «automatique ». C'est pour cette raison que diverses stratégies sont expérimentées pour contrer les assignations liées à la socialisation et au Patriarcat, illustrant le rapport dynamique qui lie principes et pratiques.

Par ailleurs, le fait que les membres de l'organisation soient sensibles à l'analyse féministe fait en sorte que sur le plan formel, du moins, les tâches ne sont pas définies selon un schéma sexué. Aucune tâche n'est vue comme féminine ou masculine :

Je pense que contrairement à d'autres groupes et $\grave{a}$ d'autres places l'avantage c'est qu'on n'arrive pas en réunion en disant "un homme ça fait ça, une femme, ça fait ça, donc faudrait que ça reste de même ». Pas mal tout le monde est assez ouvert et ouverte à l'idée qu'il faut qu'il y ait des rotations et tout ça, un meilleur partage, mais dans la pratique c'est ça on reproduit un peu malgré nous ce quion a appris et ce qu'on fait tout le temps.- Oli

Malgré l'importance de la rotation des tâches et l'introduction de certains mécanismes devant la favoriser, la majorité des militant-e-s rencontré-e-s $s$ déplorent le fait que certaines tâches soient souvent assumées par les mêmes personnes. Souvent, les tâches qui sont désignées par l'absence de rotation de tâches sont celles à haute désirabilité sociale ou celles qui sont rétribuables. 


\section{La division sexuelle du travail militant à l'UCL}

Les limites de la rotation des tâches entraînent une concentration de certaines tâches liées au pouvoir entre les mains de quelques personnes, surtout des hommes. Presque toutes les personnes interrogées reconnaissent qu'il existe une division sexuelle du travail militant dans l'organisation.

La division sexuelle du travail s'oppose à des principes centraux pour les militant-e-s de l'UCL : la rotation des tâches, l'égalité, l'éradication de toutes les formes d'oppression. Certain-e-s reconnaissent que la DSTM relève d'un rapport de pouvoir :

Pour égaliser les rapports, moi je pense à un partage égalitaire des tâches, de faire attention à la rotation des tâches. Pour éviter que justement il y ait un développement de leadership ou de rôle de domination quelconque. Antoine

Plusieurs lient la DSTM à la socialisation et aux structures patriarcales :
Je donnerais la même réponse que pour expliquer le ratio hommes-femmes débalancé. C'est-à-dire que la prise de parole et tout, ça implique, ça demande une certaine confiance en soi qui est quelque chose qu'on apprend peu aux filles, malheureusement, à cultiver: Aussi, l'aspect public-privé, encore. J'ai pas de réponse très originale à donner à ce niveau.- Maxime

La DSTM opère à travers les processus de ségrégation horizontale et verticale. Sur le plan de la ségrégation horizontale, les tâches qui sont le plus assumées par les ucéliens sont les tâches visibles: prise de parole publique (porte-parole, conférences, débats, prise du mégaphone, discours, article dans le Cause Commune), tâches liées au rythme (animation de réunion, octroi des tours de parole) et direction du mouvement (développement théorique, interventions sur le forum). Les sept hommes interviewés présentent des profils plus diversifiés, s'occupant autant de tâches logistiques et invisibles que de tâches visibles.

Les tâches attribuées principalement aux femmes concernent surtout les tâches invisibles : tâches logistiques (prendre des notes, aller chercher les Cause commune, planifier et organiser des actions, trésorerie), liées à la cohérence féministe (comité femme, dénoncer 
les rapports de pouvoir, tenir des caucus non mixtes) ou alors des tâches associées à l'extension des responsabilités domestiques (planifier la nourriture, cuisiner, héberger des militants venus de l'extérieur). Les interviewées s'occupent principalement des tâches invisibles. Celles qui ont assumé des tâches visibles ne l'ont fait qu'à de rares occasions.

Sur le plan de la ségrégation verticale, les tâches généralement occupées par les femmes jouissent d'une reconnaissance moins grande, entre autres parce que plusieurs des tâches qu'elles assument ne sont pas considérées comme du travail politique, même si les tâches liées à la dénonciation des rapports de pouvoir est intimement lié aux objectifs politiques de la mouvance libertaire qui est de vivre ici et maintenant ses idéaux. Cela a un impact sur leur «capital militant » futur, surtout si on présume que ces dynamiques sont issues de leur engagement politique antérieur; la marginalisation constante des femmes, et leur mise à l'écart des tâches attribuables font qu'elles acquièrent moins de compétences «reconnues », parce qu'elles y ont moins accès (Filleule, 2008). Elles sont donc moins « visibles »:

Deux gars qui sont membres aussi depuis aussi longtemps que moi sont considérés comme des anciens. Moi, ça fait longtemps que je suis là, mais on oublie que ça fait longtemps que je suis là. Faque tu vois que, comme, moins visible.- Gabrielle

Ceci dit, il existe des hiérarchies et des différentiels de pouvoir au sein même du groupe des hommes et du groupe des femmes. Par conséquent, les catégories « homme » et «femme » représentent des catégories hétérogènes : certains hommes se retrouvent à être plus ou moins visibles que d'autres, en vertu d'autres rapports de pouvoir, tout comme certaines femmes ont accès à une meilleure visibilité et à des responsabilités rétribuables. Le fait que les tâches visibles soient occupées principalement par des hommes occulte le fait que certains hommes sont totalement absents des tâches liées au pouvoir. Par ailleurs, le fait que certaines femmes soient plus visibles illustre le fait que la prise de pouvoir demeure un fait individuel et ne se fait pas de façon collective;

J'ai quand même espoir que les filles un moment donné vont être plus organisées dans la fédération, vont prendre un petit peu plus de pouvoir, mais collectivement, parce que là, ça reposer beaucoup sur les individus ... Émilie 
Or, les hiérarchies au sein même d'un groupe social peuvent quand même être comprises en termes de rapports de genre, si elles se déclinent sur ce registre ${ }^{26}:$ : Relationships may be among men, among women, but still are gender relations - such as hierachies of masculinity among men » (Connell, 2002: 54).

De plus, malgré les hiérarchies inter-groupes, le constat de la ségrégation occupationnelle verticale demeure pertinent: les hommes se situent bel et bien en position de quasimonopole sur les positions à statut plus élevé (Roventa-Frusumani, 2009) et exercent une autorité, une influence et un leadership, orientant ainsi le devenir du mouvement.

\section{La double/triple tâche}

Le schéma de la «ségrégation horizontale » sur l'axe visible/invisible recoupe beaucoup plus que la division formelle des tâches en réunion. Le travail invisible concerne plusieurs tâches qui ne sont habituellement pas considérées comme du travail, et qui se caractérisent donc par leur gratuité et une absence de reconnaissance. Ces tâches ne donnent pas accès à une augmentation du "capital militant». La DST sociale opère à l'extérieur de l'organisation et constitue une « contrainte externe » qui affecte la disponibilité des femmes à s'impliquer politiquement.

D'une part, les femmes assument généralement plus de tâches «domestiques », ce qui affecte leur disponibilité militante. À cet égard, plusieurs hommes sont à la fois pères et militants de l'UCL alors qu'on ne compte aucune militante qui soit aussi mère. Cela interpelle la DST « extérieure » puisqu'elle suppose qu'il est plus facile de se libérer du soin aux enfants quand on est un homme (non-monoparental). Par ailleurs, plusieurs femmes de l'UCL sont en couple avec un militant de l'UCL. Les interviewées pour qui c'est le cas mentionnent l'aider dans ses tâches. Or, ce travail est invisible:

Ça arrive souvent que je lui donne un coup de main pour préparer ses trucs.

26 Les hiérarchies peuvent également faire intervenir d'autres rapports sociaux - de classe, de « race », d'orientation sexuelle, de capacité (handicap)... 
Et c'est pas ume tâche qui paraît. Les autres ne le savent pas nécessairement, tsé que... c'est pas une tâche qui paraît. Mais du fait que je sais qu'il est rushé, ben là tu te dis que je vais prendre une heure, bon je vais pas prendre tout l'après-midi, mais j'ai le temps de prendre une heure pour t'aider: - Emilie

Outre ces tâches de soutien, d'autres tâches invisibles concernent, par exemple, l'accueil et l'intégration des nouvelles personnes (la contacter avant et après la réunion, s'assurer qu'elle s'est bien sentie, vérifier si elle a des questions).

Par ailleurs, les femmes ont plus souvent la responsabilité d'assurer la cohérence féministe dans l'organisation. En vertu de l'idée du rapport de force, il s'agit pour elles de jouer le rôle ingrat de ramener les questions de pouvoir à l'ordre du jour et insister pour la prise en compte des rapports de genre. La création du rapport de force implique un travail, une confrontation et nécessite d'assumer «les contradictions multiples liées à la mutation féministe inachevée de leurs organisations respectives» (Trat, 2006). Dénoncer des oppressions à l'intérieur de l'organisation est beaucoup plus complexe que de dénoncer celles qui ont cours dans la société, puisque cela implique de pointer des camarades du doigt, lesquels réagissent parfois avec vigueur à la confrontation. La construction du rapport de force féministe relève du défi : cela implique une vigilance, une présence (pour que les questions féministes soient intégrées, pour s'assurer que les textes soient féminisés, pour que les questions de genre soient prises en compte) et souvent, des confrontations (pour dénoncer des comportements). Ainsi, cela «les conduit très souvent à cumuler de nombreuses autres responsabilités (...) et démultiplier leur temps de militantisme pour faire face à l'ensemble de leurs responsabilités (...) pour faire reconnaître l'intérêt de leur travail de féministe. » (Trat, 2006: 152). Cela renvoie à la ségrégation verticale et à la sélection des tâches qui obtiennent le statut de travail militant. Dans ce cas, le travail des ucéliennes pour transformer les pratiques oppressives est interprété par certains militants comme des « caractéristiques féminines » et non comme des qualifications militantes (Kergoat, 2010b).

Par ailleurs, plusieurs femmes et quelques hommes indiquent que l'implication dans le 
comité femme s'inscrit dans la réflexion en termes de' double/triple tâche. En effet, l'implication dans ce comité s'ajoute au militantisme "régulier », en plus de soulever des questions en lien avec le statut «particulier » de la lutte féministe, qui, à l'UCL, se situe hors de l'intérêt général;

Un des problèmes dans le fond, c'est que quand on s'implique dans le comité femme, on s'implique pas dans les autres tâches, pis moi ça m'énerve, parce dans le fond, j'ai pas envie de laisser non plus toutes les autres tâches. En tout cas, je trouve qu'on dirait qu'on se tire dans le pied dans un certain sens, parce qu'on veut amener les luttes féministes, mais dans le fond si toutes les femmes laissent tout le reste des sujets aux gars, pis s'en vont toutes faire les affaires «de femmes» tsé entre guillemets... ben... - Gabrielle

En somme, les femmes accumulent les tâches: les tâches traditionnelles, les tâches « domestiques » à la maison, les tâches liées au pouvoir en plus des tâches «féministes » liée à la lutte pour la cohérence dans l'organisation. Par ailleurs, quelques femmes sont aussi impliquées dans le milieu féministe, ce qui leur laisse aussi moins de temps pour s'investir dans des tâches « demandantes » à l'UCL :

C'est sûr qu'on pourrait parler de la conciliation du fait d'être féministe, donc tu t'impliques aussi dans le milieu féministe... avec la conciliation travail, famille, étude, et rajoute autre militantisme par dessus. Souvent, en tant que fille, tu concilies le fait que tu fais des tâches à la maison, pis du soutien, c'est des tâches qui sont cachées. Ce que je suppose en fait, c'est que y'a beaucoup de tâches assumées à la maison, qui font en sorte que dans l'organisation politique, t'arrives. dans le public, et là t'as pu de temps parce que t'es trop loadée à la maison ... et là, tsé faut que tu t'impliques, faut que tu prennes des tâches... - Émilie

Le fait que les tâches visibles soient assumées principalement par les hommes contribue à entretenir l'image publique que l'UCL est une organisation «de gars», ce qui peut constituer une barrière à l'implication de nouvelles femmes.

Dans le fond les femmes font les tâches qui sont plus privées et les hommes font les tâches qui sont plus visibles. De sorte que de l'extérieur, l'UCL va avoir l'air d'une organisation de gars, là. - Marie-Pier

Si ça se reflète pas à l'extérieur qu'on est anarcha-féministe, ce qui va en 
rester c'est que, c'est que c'est pas anarcha-féministe. Quoi, les femmes ont corrigé les articles, et les hommes ont pris la parole? Tsé, faque je me dis, ça sera pas anarcha-féministe tant que je ferai pas cet exercice-là pis que je vais me dire: $y^{\prime}$ a plus de différence. - Eve-Marie.

En conclusion, l'attention portée sur la DSTM est fondamentale puisqu'elle permet de remettre en question le modèle du «militant asexué », extérieur aux rapports de domination. Elle rend également visible les pratiques militantes des femmes qui sont souvent accomplies sur un mode informel (entretien de la sociabilité, cohésion du groupe) et invisibilisées, puisque l'accent est mis sur les tâches publiques des hommes (Filleule, Mathieu et Roux, 2007).

\subsubsection{PRINCIPES D'ÉQUITÉ DANS LA PRISE DE PAROLE ET PRATIQUES DISCURSIVES OPPRESSIVES}

Les libertaires défendent des positions égalitaristes et antihiérarchiques quant à la participation aux discussions collectives (Goldenberg, 2010). Les entrevues confirment que pour les interviewé-e-s, l'accès à la prise de parole doit être le plus équitable possible et que les temps de parole doivent être répartis équitablement. Ainsi, une personne souhaitant s'exprimer ne devrait idéalement pas rencontrer de contraintes, et toutes les personnes seraient entendues avec la même considération. Par ailleurs, la prise de parole ne serait pas utilisée comme un outil pour asseoir une position de pouvoir.

Cependant, les commentaires des interviewé-e-s indiquent que les pratiques liées à la prise de parole (en réunion, dans les débats et dans les moments informels) permettent l'établissement et le maintien des rapports de pouvoir genrés.

Dans un premier temps, les interviewé-e-s ont insisté sur le fait que la prise de parole doit être attribuée équitablement et la communication doit se faire de façon respectueuse. Ainsi, les personnes militantes rencontrées soulignent la nécessité d'appliquer le principe de rotation des tours de parole au sein des réunions. Pour cette raison, l'alternance homme-

femme est pratiquée, tout comme le principe du nombre de tours de parole, où une 
personne qui souhaite prendre la parole pour la première fois pourra le faire avant celle qui est intervenue plus d'une fois.

Ensuite, les interviewé-e-s soulignent l'importance de recourir à des modes de communication qui favorisent des rapports égalitaires, et, à ce titre, d'éviter les pratiques discursives associées à la «langue macho » (annexe 2). Ce texte sur la langue macho, attribué à des activistes pacifistes de Philadelphie, illustre les comportements à éradiquer en matière de prise de parole.

Libertaire c'est un peu. en fait... c'est réellement la conscience de l'autre dans le désir de communiquer sans le dominer - Yann

Pour moi c'est hyper important de faire attention à ce qu'on appelle la langue macho, la manière de s'adresser aux autres, particulièrement aux femmes. De se questionner sur ses manières de faire et sa manière de prendre la parole. De ce qui est dit aussi, de pas se réapproprier à son compte ce que les femmes ont dit avant soi, de pas essaver de parler plus fort que tout le monde, déjà de juste suivre les tours de parole, de respecter les tours de parole homme-femme. Etre alerte aussi aux comportements, pas juste soi, mais autour de soi aussi.- Antoine

\section{Sous-représentation des femmes dans la prise de parole publique}

La plupart des militant-e-s ont conscience que la plupart des communications publiques, en matière de rédaction d'article pour le Cause commune, d'animation de débat, d'intervention dans une conférence, d'écriture de lettre d'opinion parue dans les médias, sont surtout prises en charge par des hommes. La question de la prise de parole est indéniablement liée avec la division sexuelle du travail.

Cette situation n'est pas exclusive à l'UCL : «Les hommes en tant que classe de sexe sont, par exemple, privilégiés dans la prise de parole. Dans un numéro publié en 2004 pour souligner le $50 \mathrm{e}$ anniversaire du journal Le monde libertaire, l'organe de la Fédération anarchiste (FA) en France, on compte trois textes de femmes pour cinquante-trois textes d'hommes. » (Dupuis-Déri, 2009 : 201). 
La dynamique à l'œuvre semble être que les hommes se proposent plus spontanément et que les femmes, plus réticentes, les laissent faire. Plusieurs associent cette dynamique aux impacts d'une socialisation différenciée, qui ferait en sorte que les femmes auraient moins confiance en leurs aptitudes politiques;

Je suis vraiment gênée, mais je me dis qu'un moment donné je ne serai plus gênée de représenter publiquement l'UCL. L'affaire, c'est que je me dis tout le temps, ah ben non, ça me tente pas, ah ben non, ça me tente pas Gabrielle

Les tâches qui sont plus réalisées par les hommes, comme d'écrire, de prendre la parole, c'est plus des gars, et c'est précisément ce que moi je fais le plus.- Maxime

Mais le fait aussi que y'en a qui est très volontaire pour aller jaser. Tsé quand tu vois que quelqu'un c'est son dada, et qui est tout heureux de le faire, ben généralement, on se pose même pas la question et on se dit, ben, ça va être lui qui va y aller. - Yann

Je montrerai plus loin qu'au-delà de la socialisation, ce sont des rapports de pouvoir qui limitent la prise de parole des femmes.

Malgré tout, quelques femmes ont pris la parole à l'occasion. Les interviewées reconnaissent qu'un effort est fait pour que les femmes soient plus visibles dans la prise de parole publique, mais que cette stratégies soulève des ambiguïtés. En effet, certain-e-s attirent l'attention sur le risque qu'une pression soit mise sur les femmes «pour que ce soit une femme qui y aille, genre : ça nous prend une femme », alors que plusieurs auraient besoin de formation et de mentorat. D'autre part, ce sont souvent les mêmes femmes qui agissent comme porte-parole, ce qui montre que les processus d'égalisation ne sont pas achevés: ce sont des femmes qui ont développé des compétences et non l'ensemble du groupe des femmes. Jusqu'à présent, malgré certains efforts collectifs, l'empowerment semble surtout une affaire individuelle, qui concerne quelques militantes.

\section{Sous-représentation des femmes dans les instances}

Les réunions et le forum sont les instances de l'UCL. C'est là où sont prises la plupart des 
décisions et où est organisé le travail. Par conséquent, le fait d'y prendre la parole et d'y être écouté-e augmente la possibilité d'influencer les revendications, les stratégies et les choix d'action.

Tout-e-s les militant-e-s reconnaissent que certaines personnes s'y expriment plus que les autres. Elles et ils ont été invité-e-s à s'exprimer sur les caractéristiques des personnes qui s'expriment le plus. Les aspects qui sont nommés le plus souvent sont le niveau de scolarité, l'âge, l'expérience militante. Le sexe/genre est généralement nommé, mais pas par tous.

Ah là, c'est drôle parce que j'avais pas pensé, mais depuis tantôt j'ai des têtes, pis ben oui, j'avais juste des gars (rires). C'est sûr que y'a des filles qui parlent plus que d'autres, mais je les avais pas en tête, en répondant aux questions - Gabrielle

À l'analyse des entrevues, on se rend compte que le genre est le facteur le plus déterminant, en ce sens que plusieurs femmes dans l'UCL combinent un haut niveau de scolarité et une bonne expérience militante, mais elles ne sont pourtant pas « visibles » :

Tsé comme moi j'en ai de l'expérience, c'est pas comme si j'avais jamais milité de ma vie. On dirait que vu que moi aussi j'ai une scolarité quand même, j'ai comme tendance à faire bon y'est intelligent, mais moi aussi je suis capable d'en dire des affaires Mais je pense qu'il y a plein de filles qui sont là qui ont une expérience militante aussi grande, mais que des fois qu'on se laisse intimider. - Ève-Marie

Par conséquent, sur le plan des tours de parole, malgré l'alternance homme-femme, la plupart des personnes interrogées reconnaissent que les femmes parlent moins que les hommes dans les instances.

Tu regardes sur le forum fédéral, tu regardes dans les 20 premières interventions, ce sont des hommes sauf peut-être une ou deux. Ça adonne que les hommes sont vraiment majoritaires dans les interventions.- Josiane

Les militant-e-s sont divisé-e-s sur cette question : certains croient que c'est parce que les femmes sont moins nombreuses, alors que la majorité reconnaît que le ratio d'intervention faites par les femmes est inférieur à leur proportion dans l'organisation. Le lien avec les 
rapports de pouvoir est tracé par certain-e-s.

Et oui, le sexe est un facteur, c'est assez évident. Le fait que les gars parlent plus est un pouvoir en soi-Maxime

Cela correspond à d'autres observations: les prises de parole en réunion sont majoritairement masculines, et les «orateurs » reconnus pour leur «charisme » sont des hommes. Par ailleurs, cela renvoie à une division entre les rôles d' «attention-getting », socialement dévolu aux hommes, et d'《attention-giving », socialement dévolu aux femmes (Bargel, 2005). Il s'agit en effet d'un prolongement des comportements sociaux : «De manière générale, les hommes - anarchistes ou non - ont tendance à parler plus souvent et plus longtemps que les femmes, à les interrompre et leur imposer les sujets de discussions qu'ils estiment importants. Les études sociologiques à ce sujet sont abondantes, montrant en outre que la parole publique des hommes obtient plus de légitimité que celle des femmes » (Dupuis-Déri, $2009: 201)^{27}$.

L'écart genré en matière de prise de parole est interprété, comme je l'ai dit plus tôt, surtout comme un effet de la socialisation des hommes et des femmes. Par contre, les rapports de pouvoir sont également déterminants dans le fait que plusieurs femmes s'abstiennent de prendre la parole.

\section{Aspect « qualitatif » de la prise de parole : des pratiques discursives oppressives}

Les entrevues ont révélé que la prise de parole est empreinte de rapports de pouvoir genrés.

Or, si la plupart des interviewé-e-s parlent des comportements qui peuvent être associés à la langue macho, ils et elles ne les associent pas nécessairement à des rapports de domination: si ces rapports ne sont pas identifiés comme constituant une oppression, il est difficile alors de pouvoir les éradiquer. ${ }^{28}$

\footnotetext{
27 Corinne Monnet (1998) a réalisé une étude fort convaincante sur le sujet.
}

${ }^{28}$ Je reviendrai sur cette hypothèse au chapitre suivant. 
Les pratiques oppressives qui sont identifiées par les ucélien-ne-s sont : couper la parole, utiliser plusieurs tours de parole et se répéter, faire des sophismes d'autorité, décourager les autres à proposer des idées, nier les sentiments, faire des interventions interminables, et recourir à des attitudes machistes et virilistes. Ce sont toutes des pratiques qui découragent les femmes à prendre la parole dans un débat. Un militant résume les pratiques qui doivent changer :

Dans toutes les choses qui ont besoin de changer, je vois toute la langue macho, toutes les attitudes de parler plus fort, reprendre les propos d'une autre à son compte, répéter ce qui a été dit comme si c'était plus important, le ton, les paroles, on le fait malgré nous sans même y penser. Ça c'est des choses qu'on devrait essayer, mais qui ne se fait pas tout le temps. Si moi je parle et que je m'emporte et que je lève le ton, de un, je devrais être capable de le réaliser, mais vu que ça marche pas tout le temps de même idéalement j'aimerais que quelqu'un soit capable de faire : heille! - Oli

À cause de cette dynamique en matière de prise de parole, toutes les femmes expriment un mécontentement quant à leurs possibilités de prise de parole ${ }^{29}$. Seule une interviewée se dit satisfaite de l'état de la prise de parole à l'UCL.

En somme, les femmes soulignent que ces rapports de pouvoir constituent des obstacles à leur propre prise de parole en réunion ou sur le forum. Les sophismes d'autorité et la rigidité idéologique constituent les types de rapports de pouvoir discursif les plus nommés : «cette tendance à utiliser les références théoriques liées à un courant idéologique pour s'approprier une position d'autorité et être intraitable sur certains sujets ou propositions » (Quirion, 2008: 114). Ceci limite la place que peuvent prendre les autres dans une discussion ou un débat:

Mais quand tu parles avec quelqu'un qui te cite des auteurs à chaque phrase, ben veux, veux pas, tu la crois ou tu prends ton trou, faque ça ça joue, là - Gabrielle

En plus de cette méthode de prise de pouvoir sur autrui, certaines pratiques discursives oppressives, liées à des attitudes virilistes, contribuent à tenir les femmes (et les hommes

\footnotetext{
${ }^{29}$ Goldenberg (2010) fait le même constat.
} 
qui refusent de jouer le jeu du pouvoir) à l'écart de la prise de parole.

$Y^{\prime} a$ des individus dans l'UCL, c'est pas tout le monde, mais qui ont des attitudes assez viriles pis machistes, dans leur façon d'intervenir. Les combats de coqs, ça existe. Pis on voit ça. Pis c'est sûr que ça ne donne à personne l'envie d'intervenir. Mais c'est sûr que le fait que les femmes soient pas socialisées pour prendre la parole de la même façon, à aller déchirer sa chemise. Mais c'est pas si pire que ça, on s'entend. - Pierre-Luc

La littérature relaie l'idée que les rapports de pouvoir limitent la possibilité de contribution des femmes. Plusieurs militants valorisent un style d'intervention plutôt «viril » et belliqueux (Brouillette et coll., 2010) qui leur semble en adéquation avec «l'esprit de la lutte ». Ces militants résistent à une «expertise » de communication mise en pratique dans les milieux féministes, en les interprétant comme des «caractéristiques féminines » et non comme des qualifications militantes permettant une meilleure adéquation entre les principes et les pratiques (Kergoat, 2010).

Par exemple, une militante rapporte une discussion avec un militant à propos de la façon de clarifier ses malaises, d'exprimer des désaccords politiques ou personnels. Or, son vis-à-vis a ridiculisé ce mode de communication, qu'il a taxé de «féminin ». Dans l'optique où les libertaires souhaitent mettre en place des processus égalitaires, ces modes de communication, visant à éradiquer les rapports de pouvoir et les oppressions dans la communication, devraient être considérés comme un travail politique.

Pour sa part, Monnet (1998) propose une approche en termes de division du travail de conversation, en vertu duquel les hommes s'attachent à contrôler la conversation à leur avantage (initiative et contrôle) et relèguent les femmes à des tâches de soutien. Ils utilisent des pratiques qui découragent les initiatives des femmes pour introduire de nouveaux sujets ou pour prendre l'initiative de la conversation (couper la parole, ne pas reprendre les sujets apportés par les femmes, revenir constamment à son idée, ne pas montrer de signe d'attention ou d'intérêt). Ces pratiques participent à l'établissement et au renforcement de la hiérarchie (Monnet, 1998). 
Ces pratiques sont identifiées par presque toutes les personnes rencontrées en ce qui concerne les réunions et les espaces formels. Or, lorsqu'on parle des espaces informels, ces rapports de pouvoir dans la conversation deviennent beaucoup plus visibles. Les interviewé-e-s racontent qu'elles se font souvent couper la parole et peinent à intégrer les conversations enflammées à très forte voix, animées par les hommes. En somme, les femmes considèrent que les pratiques oppressives limitent leur accès à la prise de parole, tant publique, au sein des réunions que dans les conversations informelles. Les hommes identifient majoritairement le facteur de la socialisation.

\subsubsection{TRIPLE OPPRESSION, ANARCHA-FÉMINISME ET HIÉRARCHISATION DES LUTTES}

La plate-forme organisationnelle de l'UCL est largement inspirée de celle de la NEFAC. Cette plate-forme, qui constitue la base d'unité théorique, mentionne que l'UCL se réclame de l'anarcha-féminisme:

"L'anarcha-féminisme est une théorie et une pratique par laquelle nous
critiquons et attaquons le triple règne du Patriarcat, du Capitalisme et de
l'État. C'est seulement en unissant la perspective révolutionnaire de classe
de l'anarchisme et la critique féministe du Patriarcat que le féminisme et
l'anarchisme peuvent atteindre leur but commun de libération humaine. "Il
n'y aura pas de révolution sans libération des femmes. Il n'y aura pas de
libération des femmes sans révolution." " ${ }^{30}$.

Dans cette section, je montrerai à quel point les personnes interviewées jugent souhaitable (ou pas) la réflexion en termes de triple oppression et comment cette réflexion simultanée est mise (ou pas) en pratique dans les actions, les publications et le développement théorique.

\section{Consensus relatif sur la question de la simultanéité des oppressions}

Toutes les interviewées acceptent l'idée que les oppressions capitaliste et patriarcale peuvent être réfléchies simultanément et qu'elles sont d'égales importances dans l'analyse.

\footnotetext{
${ }^{30}$ Site web de l'UCL, Buts et principes [En ligne] http://www.causecommune.net/presentation/buts-etprincipes
} 
Chez les hommes, les propos sont plus polarisés sur la notion de simultanéité des oppressions et d'articulation des luttes. Certains avancent que le lien entre la lutte de classe économique et le féminisme est évident et impossible à remettre en question, alors que d'autres mentionnent ne pas vouloir prioriser une lutte plutôt qu'une autre, mais appellent néanmoins à douter de la possibilité théorique et pratique d'associer ces oppressions:

Les analyses plus profondes et plus réfléchies et je sais pas à quel point estce que le féminisme radical a été capable, ou est capable d'avoir un espèce de renouvellement comme ça, que le marxisme a eu. - Antoine

Plusieurs militants ignoraient que la plate-forme de l'UCL comprenait une position anarchaféministe, tandis que pour d'autres, la notion reste floue, notamment à cause de la difficulté à établir des nuances claires d'avec le féminisme radical et matérialiste. Entre autres, un militant a déploré le fait que cette position ne soit pas plus connue et diffusée.

Selon quelques militant-e-s plus ancien-ne-s, cette méconnaissance est liée à plusieurs facteurs. Par exemple, lors de la refondation, la plate-forme de la NEFAC a été adoptée presque intégralement, et aucune discussion collective ne s'est tenue sur cette question.

Ça c'est une question que je trouve qu'on n'a pas vraiment poussé la discussion de dire : qu'est-ce que ça veut dire pour la majorité du monde. C'est l'impression que j'avais eue à l'époque et que pour ben du monde aussi c'est que anarcha-féministe, ça sonne bien faque on n'a pas de problème à se dire de même, mais la réflexion a pas été vraiment plus poussée que ça.Oli

Enfin, les personnes interrogées remarquent que la question de la triple oppression fait généralement consensus et que, sauf exception, personne ne la remettra ouvertement en question. Par contre, malgré une reconnaissance formelle de la simultanéité des oppressions, elle leur paraît moins ancrée dans la pratique, notamment en matière d'actions, de thèmes abordés dans les publications et de développement théorique, où plusieurs identifient une hiérarchisation des luttes, au profit de la lutte anticapitaliste.

\section{Triple oppression et revendications/actions}

Les militant-e-s de l'UCL organisent des actions militantes qui visent à porter leurs 
revendications dans l'espace public. Lorsqu'interrogé-e-s sur celles-ci, ils et elles identifient surtout des actions qui touchent les rapports de classe : participation au G20, campagne contre le budget provincial, manifestation contre le dégel des frais de scolarité, participation au ler mai, canular dénonçant Charest, participation à la manifestation contre la brutalité policière. Parallèlement, la plupart des militant-e-s reconnaissent que peu d'actions portent directement sur les questions antisexistes, féministes ou contre le Patriarcat.

De leur côté, les militant-e-s du collectif de Québec considèrent que les actions qui touchent les rapports de genre et le féminisme sont assez présentes au sein de leur collectif, ayant pris la relève des vigies pro-choix suite à la dissolution du collectif féministe non mixte qui se chargeait de l'action.

En fait, si les actions portant spécifiquement sur les rapports de genre sont jugées peu présentes, la perspective de genre est rarement intégrée aux autres actions :

\footnotetext{
Mais en même temps, je ne suis pas certain qu'on intègre toujours l'analyse féministe dans les critiques qu'on fait, dans les actions qu'on fait. Je pense que là, c'est plus difficile des fois. La perspective de classe ressort toujours. La perspective féministe, pas nécessairement. C'est plus, c'est quand même un peu plus secondarisé, je pense. - Pierre-Luc
}

Le fait que les principales actions concernent l'opposition au capitalisme ou à l'État révèle une hiérarchisation des luttes, dans ce cas, dans la sélection des actions et revendications. C'est l'une des manifestations des rapports de genre dans l'UCL, où la préséance des hommes octroie un statut «universel » ou «général » à la lutte des classes. Le groupe dominant s'assure que les demandes des autres groupes demeurent «particulières ». Par ailleurs, les rapports de pouvoir, qui écartent les femmes de la prise de parole, font que celles-ci ont moins d'impact sur la sélection des revendications et actions. En somme, cela supporte « [...] l'hypothèse que les revendications [sont] déterminées par l'état des rapports sociaux de sexe au sein du mouvement et qu'elles concernaient davantage les hommes» (Dunezat, $1998: 180$ ). 
Enfin, toutes les militantes rencontrées identifient et déplorent la sous-représentation des thématiques féministes qui traduit la hiérarchisation des luttes.

Tu peux pas manquer le premier mai, mais le 8 mars oui. Les actions plus lutte de classe vont passer avant les actions féministes- Josiane

Certains hommes croient que l'initiative des actions féministes doit venir des femmes ellesmêmes. D'ailleurs, le comité femmes est né, entre autres, du désir des femmes d'octroyer une plus grande place aux questions et actions féministes dans l'organisation. Selon elles, le comité femme joue néanmoins un rôle ambigu : les questions de genre y restent cantonnées et isolées de la responsabilité collective, avec, donc, un statut de «question à côté ». Cette façon de faire est d'ailleurs assimilée par un militant à un rapport de pouvoir :

Je trouve justement que la question du comité femmes a justement été faite dans un rapport de pouvoir, de nous on veut pas en parler, on veut parler de notre comité action ouvrière... voilà. Je donne l'exemple du comité action ouvrière, mais ça aurait pu être n'importe quel autre comité... - Yann

Le fait de taire les questions de genre lors des actions est assimilé par quelques militants à un rapport de pouvoir : qui participe à la sélection des revendications? Le rapport entre les groupes de sexe permet d'expliquer la sélection de certaines revendications plutôt que d'autres (Dunezat, 1998).

Bref, c'est ça antssi les relations de pouvoir dans les associations, c'est qu'est-ce qui va être mis à l'agenda politique. Pis ça, c'était pas en haut de l'agenda politique, clairement.- Raphaël

Quand quelqu'un dit à quelqu'un d'autre: ah ça c'est une affaire de femmes, vous en parlerez au comité, toutes les affaires féministes vous vous en parlerez au comité femmes. Je trouve que c'est un rapport de domination de genre, de dire : ah ben ça m'intéresse pas, vous en parlerez ailleurs- Yann

Par contre, des hommes relativisent la hiérarchisation des luttes dans les actions. Tous vont remarquer le peu d'actions strictement féministes, ou la rare intégration de l'analyse de genre dans les « autres » actions. Ils insistent plutôt sur l'effort qui est fait pour intégrer ces questions et sur le fait que le processus qui mène à ces actions est féministe (recours à des processus égalitaires de prise de décision). 
Est-ce que les personnes interviewées jugent que les principes féministes de l'organisation sont bien représentés dans les actions? Les femmes répondent spontanément par la négative alors que les hommes y vont d'un « oui et non ».

Certaines actions féministes ont lieu. Le collectif de Québec est reconnu pour ses participations aux contre-manifestations pro-choix. À Montréal, c'est la création et la distribution de l'autocollant «Mon cul n'est pas une marchandise » et tous les débats sur le sujet de la prostitution, tant au sein de l'UCL qu'avec d'autres organisations féministes et/ou anarchistes, qui retiennent l'attention. Ces vifs débats ont opposé les approches du travail du sexe et celles de l'exploitation sexuelle. La création et distribution de l'autocollant a été identifié par plusieurs comme une action féministe, qui de plus, intègre des réflexions liées à la lutte des classes, malgré l'absence de consensus sur la position à tenir sur la question de la prostitution.

Enfin, l'éviction ou la sous-représentation des thématiques féministes dans les actions de l'UCL peut être liée à une question que j'ai abordée plus tôt. II s'agit du fait que l'UCL est organisée avant tout autour d'un rapport social considéré prioritaire, soit le rapport de classe. Si les militante-e-s reconnaissent l'aspect lutte-de-classiste de leur organisation, la dimension féministe de l'UCL fait moins l'unanimité. Les questions qui touchent les rapports de genre sont généralement éclipsées au profit des thèmes récurrents de la lutte des classes.

\section{Triple oppression et publications}

Le même phénomène survient en ce qui concerne les publications, comme la rédaction du Cause Commune, qui constitue une activité récurrente et incarne le visage public de l'UCL. En matière de message collectif, on constate que les questions féministes y trouvent une diffusion moins importante que les questions de travail ou de syndicalisme (voir annexe 3).

En effet, les militant-e-s constatent que peu d'articles féministes sont publiés et que 
l'analyse féministe est peu intégrée aux « autres » sujets de façon transversale. Là encore, l'analyse que les militant-e-s font des publications révèle une tension entre les principes liés à la simultanéité des oppressions et la pratique militante où ces luttes sont en fait hiérarchisées au bénéfice de la lutte des classes.

Mais moi ce qui me pose problème c'est le fait qu'il y ait des dossiers «femmes» pis des dossiers comme normal. Alors comme je disais pour moi, ça devrait être une espèce d'analyse transversale, tsé plutôt qu'un truc à côté, ça devrait être ... pas à chaque fois, mais on devrait toujours avoir ça un petit peu dans le discours, de le ramener: - Antoine

Toutes les femmes reconnaissent que les principes féministes sont peu présents dans les publications, sauf lors du 8 mars. Certaines notent que souvent, il n'y a aucun article féministe ou intégrant la question des rapports de genre dans le Cause Commune. D'ailleurs, les femmes contribuent moins à la rédaction. Celles qui le font ne veulent pas nécessairement n'écrire que des articles féministes.

Comme pour la question des actions, les hommes nuancent l'idée de la hiérarchisation et tendent à mettre l'accent sur le fait que beaucoup d'efforts sont faits pour augmenter le nombre de textes féministes dans les publications, même s'ils reconnaissent qu'il y a encore matière à amélioration.

\section{Triple oppression et positionnement théorique}

L'un des aspects importants du travail de l'UCL est de contribuer au développement théorique de notions libertaires et à effectuer des analyses de la conjoncture politique. En ce qui a trait aux débats et aux discussions entourant le développement théorique, les militante-s interrogé-e-s mentionnent souvent l'éviction des positions féministes. Plusieurs remarquent la sous-participation des femmes aux activités de développement théorique, ce qui a certainement un lien avec le premier aspect, et avec les rapports de pouvoir genrés.

Comme l'identifie Delphy, «Selon plusieurs, les femmes sont opprimées, mais pas exploitées comme les prolétaires, donc, leur question est secondaire, ce qui se traduit par 
une négation par plusieurs de la dimension économique de l'oppression des femmes » (Delphy, $2001: 8$ ). Cette subtile distinction théorique entre exploitation et oppression fait l'objet de certains débats à l'UCL. En effet, certains refusent de considérer le groupe des femmes comme une classe exploitée au même titre que les travailleurs, ce qui correspond au malaise que certains marxistes plus orthodoxes (qui ont été et sont toujours présents dans l'extrême-gauche en général, et à l'UCL en particulier) entretiennent avec le féminisme et la notion de triple oppression. Ces militants insèrent des nuances théoriques afin de distinguer les oppressions, au nom de l'unité du prolétariat :

Je sais pas, j'ai trouvé chez mes camarades féministes cette tendance un peu à plaquer lutte de classe sexe/genre, lutte de classe bourgeois -prolétaire comme un peu la même chose, je pense qu'il y a des différences essentielles entre les deux, et je pense que ça peut être un point de départ, un espèce de lieu de repère sur lequel on peut s'appuyer pour aller plus loin, mais pour moi il y a une différence importante. Ça veut pas dire qu'on secondarise une question ou une autre, absolument pas. - Maxime

J'ai mentionné plus tôt comment cette «rigidité idéologique » agit comme rapport de domination. Elle relève à la fois d'une pratique oppressive en matière de prise de parole et comme révélateur de la hiérarchisation des luttes à l'UCL.

Pis sur les autres rapports de pouvoir en général, un peu: $y^{\prime} a$ un groupe de personne qu'on appelle les "redneck», dans l'UCL, c'est comme ceux qui sont plus comme rouges que noirs et qui sont plus autoritaires, pis plus déterministes de classes, et plus lutte-de-classistes. Et quand ils parlent, leur parole a plus de portée que les autres. Ça serait peut-être basé sur l'expérience, et c'est pas surprenant, mais tout les gens qu'on appelle les redneck, c'est des gars. Des gars, vieux, avec beaucoup d'expérience.Marie-Pier

D'avoir des grandes discussions sur les luttes de classes, le travail, le rapport au travail, en parlant jamais de Patriarcat. Pis un moment donné t'a le goût de dire, ouais pis le Patriarcat là-dedans? Mais tsé, vu que c'est des discussions super universitaires et tout ça, je suis sûre qu'il y a des gens qui comme... ont pas envie de confronter - Eve-Marie

En somme, les militant-e-s, surtout les femmes, remarquent que l'analyse «intersectionnelle» est peu mise de l'avant au sein des actions, des publications et du 
développement théorique à l'UCL.

Par ailleurs, les femmes qui prennent la parole introduisent une analyse qui insiste davantage sur la critique du Patriarcat. Cet intérêt des femmes pour le féminisme dans une organisation mixte comporte un effet pervers; le risque d'invisibilisation : « les femmes, en introduisant une voix différente de celle des hommes dans les mouvements sociaux, courent le risque d'être moins entendues, à la fois par leurs pairs masculins, mais aussi par les analystes » (Filleule, Mathieu et Roux, 2007).

En somme, les militantes constatent une «dissymétrie structurelle qui est simultanément l'effet et le garant de la domination : l'un se pose comme le représentant de la totalité et le seul dépositaire de valeur et de normes sociales imposées comme universelles parce que celles de l'autre sont explicitement désignées comme particulières » (Hirata et coll., 2004: 44-45). La pensée anticapitaliste secondarise souvent la pensée antipatriarcale via «la centralité et la primauté des rapports de classe » dans la compréhension des relations de pouvoir (Blais et Courcy, 2010). Les féministes de l'UCL remettent cette priorisation en question, avec un succès mitigé, puisque les questions et actions féministes demeurent souvent «en plus et à côté ». Ce phénomène interpelle à la fois la division sexuelle du travail (les réunions du comité femmes, l'accès à la prise de parole) et la question de la confusion du masculin avec l'universel.

\subsubsection{LE PRINCIPE DU « PRIVÉ EST POLITIQUE » ET LES RAPPPORTS DE POUVOIR DANS LES ESPACES INFORMELS}

L'inclusion du principe du « privé est politique » dans la plate-forme organisationnelle a été proposée par une militante présente au congrès de refondation à l'automne 2008 (quand les collectifs québécois se sont dissociés de la NEFAC ${ }^{31}$ pour former l'UCL). Ce principe postule l'absence de distinction entre sphère publique et sphère privée et soutien que cette

\footnotetext{
${ }^{31}$ La NEFAC (North-est Federation of Anarchist Communist) est une organisation qui regroupait des collectifs du Nord-est des États-Unis, l'Ontario et le Québec. Suite à la refondation, les collectifs du Québec se sont séparés et regroupés dans I'UCL.
} 
dichotomie est instituée par la domination masculine et appelle à ce que les militant-e-s politisent leurs actes individuels, même à l'extérieur de l'organisation ${ }^{32}$.

Les interviewé-e-s savent que ce principe a été adopté et en connaissent bien les tenants et aboutissants. Les personnes qui ont abordé la question l'ont d'ailleurs fait spontanément, puisqu'aucune question du guide d'entrevue ne portait sur ce principe. Cette mention spontanée me porte à penser que l'adoption représente un événement important, à la fois révélateur de la dynamique des rapports de genre de l'époque, et signe d'une volonté de changement.

Tout-e-s les militant-e-s (sauf un homme et une femme) considèrent que leurs principes égalitaires et anti-oppression devraient nécessairement se manifester autant dans leur pratique militante que dans leur vie personnelle. Selon elles et eux, il s'agit d'une question de cohérence avec les idéaux libertaires, c'est-à-dirre de vivre ici et maintenant les principes qui guident leur vision d'un monde post-révolutionnaire; des relations humaines égalitaires, non oppressives et non hiérarchiques tant au sein des activités formelles de l'UCL (réunions) que dans les relations entre militant-e-s et avec des non-militant-e-s.

Etre libertaire, ça signifie prendre tout le temps en compte les rapports de force, les rapports de domination potentielle et les neutraliser avant qu'ils se produisent. Ça c'est tannant, mais c'est comme ça. Je pense que c'est probablement l'aspect le plus concret d'être libertaire en société. C'est tout le temps d'être en mode d'analyse - Hugo

De plus, les militants (2) qui abordent la définition du "privé est politique » offrent une définition conforme à ce que mentionne la plate-forme organisationnelle.

\footnotetext{
32 Voici la proposition adoptée au congrès: "Les membres de l'UCL s'engagent, en tant que communistes libertaires, à renier la dichotomie entre la sphère publique et la sphère privée historiquement instituée pour la domination masculine et largement propulsée par l'État noderne. Les membres de l'organisation s'entendent donc sur la nécessité de politiser les actes individuels, même à l'extérieur « formel » de l'organisation, puisqu'il importe de nous responsabiliser en tant qu'individus dans l'ensemble de nos choix politiques. Reconnaître que le privé est politique, c'est par le fait même reconnaître les oppressions sociales, économiques et politiques fondées sur la sexualité, le sexe, le genre, la couleur de la peau, l'origine ethnique et autres soient partie prenante de notre lutte pour le projet communiste libertaire. " http:/www.causecommune.net/presentation/constitution\#Le priv est politique
} 
C'est reconnattre qu'en dehors de l'organisation et dans nos vies privées ça continue et que l'UCL en tant qu'organisation s'attend à ce que chaque membre ou que chaque sympathisant et sympathisante applique un peu ses principes en dehors des réunions, des manifs et compagnie. Oli

En somme, les militant-e-s connaissent bien le principe du «privé est politique », sa signification et sont en accord avec l'idée que les principes portés par l'UCL doivent également se retrouver dans leur vie privée.

\section{L'adoption du principe vise à réagir à certaines situations}

Pour les femmes, surtout, l'introduction du principe «le privé est politique » marque une rupture avec l'approche de la NEFAC concernant les rapports de genre, en ce sens qu'elle met en lumière le choix collectif de formaliser les principes féministes dans sa constitution et de ne pas tolérer des comportements contraires à l'éthique libertaire, tant au sein de l'organisation politique qu'au sein de la vie quotidienne des individus. Plusieurs jugent qu'il s'agit d'un signe que la question féministe est maintenant intégrée dans les principes (discours public) de la nouvelle organisation (UCL).

Ainsi, le «privé est politique » est considéré, par les interviewé-e-s, comme un principe important et nécessaire dans une pratique anarchiste et féministe.

On a officiellement rentré une position féministe dans la plate-forme, alors pour moi c'était comme on officialise un peu le fait qu'on soit féministes. La NEFAC, je sais pas, j'aurais peut-être dit... c'est sûr qu'il y avait des individus qui étaient féministes, mais pour moi, inclure que «le privé est politique », c'était le point qui était important.-Gabrielle

L'inclusion du "privé est politique » constitue, en quelque sorte, une réaction des femmes militantes (et de certains hommes) à la difficulté d'expulser des militants suite à des situations de violence. Les femmes voulaient camper le fait que certaines situations sont inacceptables, surtout chez des individus qui se disent libertaires.

Y'a eu des histoires d'horreur, aussi, à l'intérieur même de l'UCL, et d'anciennement la NEFAC. Des histoires de couples qui ont mal toumé, des trucs assez trash. - Pierre-Luc 
En même temps, y'a un gars qui a été expulsé du collectif pour violence conjugale et des histoires avec d'autres femmes de la fédé. J'avais ramené cette histoire au congrès pendant les discussions, et comme ça je me disais, grâce à ce cas-là. la proposition de la camarade, elle va passer. Coûte que coûte - Tanya

\section{Les débats qui entourent l'adoption du principe «le privé est politique »}

Toutes les personnes interviewées qui abordent le privé est politique mentionnent le contexte d'adversité qui a entouré le débat de cette proposition lors du congrès de refondation, où quelques militants ont dénoncé et ridiculisé la proposition dans le but d'en éviter l'adoption. Les femmes étaient, pour leur part, toutes en faveur de la proposition et ont dû la défendre.

Or, l'opposition de ces militants n'est pas interprétée de la même façon par les hommes et les femmes interviewé-e-s. Si certains (exclusivement des hommes) présument qu'une mauvaise interprétation ou une incompréhension de la notion du privé est politique est en jeu, d'autres (en particulier les femmes) supportent la thèse de la mauvaise foi et du rapport de pouvoir :

Il faisait exprès. Y'est pas con, ce gars-là, il essayait de ridiculiser la proposition.- Josiane

D'autres personnes, sans s'opposer au principe en soi, ont désapprouvé le fait que le privé est politique soit intégré à un endroit aussi important que la plate-forme organisationnelle.

Par contre, «pour l'organisation, ça allait de soi » (Antoine) que la proposition était pertinente et importante, et ce n'est qu'une minorité de militants qui y a résisté. Le débat n'a certes pas donné lieu à un schisme, mais le climat de tension a poussé des femmes qui prenaient rarement ou jamais la parole à défendre publiquement la proposition. Par la suite, elles ont invité les femmes du collectif féministe non mixte Les Sorcières à venir donner un atelier sur le sujet afin de démystifier la question. Cela illustre que les questions féministes ne font pas l'unanimité. 
Or, malgré le fait que la résistance soit le fait d'une minorité d'individus, cet épisode soulève la passion chez les gens qui le racontent. Dans le champ.lexical, on retrouve des mots comme : malsain, grandes gueules, ridiculiser, monopoliser, démagogie, exploser. Les femmes sont plus nombreuses à aborder la question, et en parlent plus longuement.

Toutefois, certaines personnes concluent néanmoins que les oppositions n'étaient « pas dues à de la mauvaise foi » ou que «à part ça, le débat était quand même correct »; les interprétations qui renvoient ces comportements à des rapports de pouvoir sont plus rares ${ }^{33}$. Plusieurs semblent considérer le tumultueux débat comme un «épisode » qui ne les empêche pas de concevoir l'UCL comme un lieu égalitaire.

\section{Les limites d'application du « privé est politique » : pas dans les espaces informels}

Dans les réunions et les activités, plusieurs mécanismes et stratégies sont mis en place pour amenuiser la distribution inégale du pouvoir (tour de parole, alternance homme-femme, moments pour aborder les malaises). Bien qu'imparfaitement appliquées dans les réunions (j'y reviendrai dans une autre section), ces mesures existent pour rendre les processus cohérents avec les principes égalitaires et non hiérarchiques de l'UCL.

Or, l'absence de ces mécanismes et de «surveillance » dans les espaces informels fait en sorte que plusieurs femmes constatent que dans la «vraie vie », autour d'une bière ou dans leurs rapports de camaraderie, les principes d'égalité leur paraissent mis de côté. Selon elles, les solutions «techniques » appliquées au cadre formel des actions et des réunions assureraient une certaine égalisation, mais non ce changement culturel qui en viendrait à octroyer aux militants une plus grande conscience envers les rapports inégalitaires qu'ils perpétuent à reproduire, et surtout, à les amener à modifier leurs comportements, sans y être contraints par un mécanisme.

On se disait qu'on avait un problème quand on était les membres du collectif, dans un party ou autour du feu, pis là, c'est comme si les principes d'égalité homme-fernme prenaient le bord parce qu'on n'était plus... faque

\footnotetext{
${ }^{33}$ C'est d'ailleurs l'objet du prochain chapitre.
} 
là on se permettrait de faire des jokes sexistes pis toute, pis on se permettrait de se couper la parole parce qu'on était plus dans un cadre formel, donc c'est pour ça que je dis que y'a comme un peu un dérapage. Gabrielle

Tsé c'est ben beau les réunions où on met des trucs et des mécanismes... pour, justement, aller chercher l'égalité, mais à l'extérieur, dans les relations interpersonnelles... Là, on dirait que toutes les barrières tombent, toutes les jokes sont permises, et tous les ... ben pas tous les comportements sont permis, mais c'est plus lousse. On dirait que là, c'est pu l'UCL, c'est une gang d'ami-e-s qui peut faire à peu près tout ce qu'il veut-Josiane

Étant donné la différence d'expérience vécue par les militants et les militantes dans les espaces informels, leur rapport à ces espaces n'est pas le même. En effet, les femmes jugent que les espaces informels représentent le lieu privilégié d'expression des rapports de pouvoir. Libérés du cadre formel de l'organisation politique et des mécanismes de gestion des rapports de pouvoir nommés plus haut, les principes d'égalité hommes-femmes leur semblent mis de côté. Pour ces raisons, elles privilégient les espaces formels pour aborder les questions de pouvoir, à travers des mécanismes institués comme la clarification des malaises, ou la présentation publique des conclusions suivant un caucus non mixte. Elles s'étonnent souvent du peu de réceptivité des hommes lorsque les questions de pouvoir sont abordées en réunion.

A contrario, les hommes se sentent plus à l'aise dans les espaces informels pour aborder leurs malaises et conflits. En ce qui les concerne, ils trouvent humiliant et confrontant de voir leurs comportements dénoncés publiquement. Par conséquent, ils privilégient la discussion informelle, plus privée, comme stratégie de gestion des rapports de pouvoir. Par conséquent, les interventions du type «boyswatch » s'effectuent principalement à travers une discussion informelle ou via le forum.

On est plus informels dans notre façon de l'approcher, moi j'ai plus tendance à aller après la réunion, mais jamais en face de tous. Je pense que y'a pas toujours nécessairement le besoin de faire ça public. Je sais pas, des gens qui vont se voir à la bière après et qui vont faire: j'ai pas aimé ton intervention. Et là ça se discute de un a un, et ça fait du bien des fois de pas être flagellé en public.- Yann 
Les gens se côtoient aussi beaucoup dans l'informel, ça peut faire en sorte aussi que c'est un autre vecteur de communication. Des fois c'est moins "dans ta face " que de en pleine réunion se lever et dire heille, hostie ça a pas d'allure, ça a pas de sens qu'est-ce que tu viens de dire. D'un autre côté si tu vas boire une bière après, tu peux.... Je sais pas si c'est mieux dans l'informel ou d'aborder ça publiquement et politiquement... ça reste à voir, mais entre ça pis rien, je pense que c'est mieux de l'aborder dans l'informel. - Antoine

Certes, les femmes et les hommes gèrent différemment les conflits (Kergoat, 2010), mais au-delà de ce constat gisent des explications qui renvoient à la dynamique interne plutôt qu'à un mode «naturel » ou à une préférence «culturelle ». En effet, les femmes parlent de la sphère informelle comme étant insécurisante, tandis que les hommes s'y sentent à l'aise. Pour ces raisons, les femmes seront réticentes à aborder les rapports de genre en informel, préférant recourir aux mécanismes institués, tandis que les hommes auront tendance à privilégier cette voie.

\section{Les rapports de pouvoir dans les couples}

En matière de privé et politique, nombre de militant-e-s abordent spontanément la question des couples dans l'UCL. En effet, la plupart des femmes de l'UCL sont en couple avec un membre. Des rapports de pouvoir observés au sein des couples se trouvent aux zones de confluence entre le couple (privé) et l'organisation politique (public). Cela interpelle la notion du «privé est politique », puisque l'abolition de la distinction entre privé et politique ne semble pas totalement achevée dans ce cas. Les interviewé-e-s se demandent si et comment intervenir, quand, par exemple :

- c'est plus souvent l'homme du couple qui est interpellé pour donner son opinion

- c'est souvent la femme qui quitte l'organisation suite à une rupture

- rupture amoureuse entre deux membres dans laquelle le collectif doit s'impliquer.

- les femmes font souvent des tâches (invisibles) pour aider leur copain dans une tâche qui octroie un crédit public

- l'homme utilise des pratiques discursives oppressives avec sa partenaire.

- les rapports de pouvoir du couple se répercutent dans les réunions et les activités : Faut prendre parti et intervenir? Est-ce condescendant? Est-ce que c'est 
paternaliste? Est-ce un manque de reconnaissance des capacités de la femme à se défendre elle-même?

Par exemple, des militants réunis lors d'un souper de leur collectif ont remarqué un rapport de pouvoir au sein d'un couple présent. S'ils ne sont pas intervenus sur le champ, les hommes ont toutefois discuté de leur malaise par la suite et ont tenté de trouver une stratégie pour aborder ces problèmes lorsqu'ils se présenteront à nouveau.

Et là, c'était comme on disait tantôt, les gars, personne intervenait, tout le monde était comme... ok, qu'est-ce que je fais? Pis y'a fallu qu'on s'en parle après le souper quand on était juste trois-quatre gars pis qu'on se dise, moi j'ai un malaise là-dessus, moi aussi, qu'est-ce qu'on pourrait faire. C'est un processus de... on se forme à travers ça, la prochaine fois je vais être plus alerte pis je vais être plus apte à intervenir sur le coup. - Raphael

Par ailleurs quelques militants avouent que c'est important pour eux, même si ce n'est pas toujours facile, d'assurer un partage égal des tâches domestiques avec les femmes qui les entourent.

C'est ben beau d'aller à des réunions de trucs féministes nananan pis d'en jaser, pis là je rentre chez nous pis c'est ma copine qui torche la salle de bain plus souvent que moi, pis des trucs comme ça que à long terme j'essaie d'appliquer ça. Pis y'a comme toute la partie individuelle, pis dans les relations avec les autres, d'assumer ce que je peux prendre comme place pour que ce soit mieux partagé et tout ça. - Oli

Moi je trouve que même moi aussi à ce niveau-là j'ai du travail à faire dans des relations..., comme mettons à la maison, que je fais encore peu ou pas, comme préparer les repas je trouve ça difficile, tsé j'en fais de temps en temps, mais c'est tout le temps la même bouffe que je fais. Faque tsé, c'est des trucs qui sont exigeants.- Raphael

En somme, l'apparente contradiction entre le principe du «privé est politique » et la pratique militante n'est pas totale. Les militant-e-s de l'UCL posent certains gestes pour assurer la cohérence en ce sens, ce qui montre que ce concept demeure un principe qui guide la réflexion et l'action, avec un degré de succès mitigé.

Comme pour tous les principes, il existe une position officielle (le privé est politique) qui se traduit dans le discours public, mais qui se retrouve intégrée et mise en œuvre à des degrés 
divers chez les militants. L'application du principe dans la pratique n'est certes pas automatique, mais les personnes interviewées insistent sur la notion de processus, ou de travail en ce qui concerne l'apprentissage de l'application des principes de l'organisation à sa vie privée.

Par ailleurs, le grand nombre de couples au sein des collectifs complique la dénonciation des rapports de pouvoir, puisque les femmes trouvent difficile de dénoncer publiquement les comportements de leur amoureux. Pour sa part: « l'homme anarchiste est trop souvent solidaire à l'extérieur du couple, envers les immigrants, les chômeurs, mais il y a un manque de solidarité dans le couple » (militante citée par Dupuis-Déri, 2009: 203).

\section{CONCLUSION}

Ce chapitre a permis d'illustrer que les principes centraux et les motivations individuelles à l'engagement dans l'UCL sont principalement articulées autour des principes anticapitalistes et anti-oppression. Par contre, pour les femmes, les rapports de genre et le féminisme sont importants, voire centraux à leur présence dans l'UCL. Le rapport social prédominant se retrouve autant dans les motivations individuelles à l'engagement qu'au sein du message collectif qui transparaît dans l'espace public. Ceci démontre que, bien que toutes les personnes rencontrées se revendiquent d'un féminisme radical /matérialiste, l'UCL est structurée autour d'un rapport social prédominant : celui des rapports de classe. Les rapports de genre y trouvent une importance qu'ils n'ont pas dans d'autres milieux, mais demeurent souvent secondarisés. Ceci pourrait illustrer la difficulté des mouvements sociaux à se structurer autour de plus d'un rapport social (Dunezat, 2007).

Plus loin, j'ai illustré les points de tension entre les principes défendus et les pratiques militantes et je les ai liés à des rapports de pouvoir, qui opèrent tant au niveau du processus que des résultats. En effet, mon approche théorique considèrent que les inégalités entre groupes sociaux sont issues de rapports de pouvoir, tout comme les relations sociales (interindividuelles) qui (re) produisent ces inégalités. Par conséquent, les différentes zones 
de tension identifiées dans ce chapitre sont assimilables à des rapports de pouvoir, et ce, même en l'absence de pouvoir coercitif ou formel, assignant de facto certaines positions à certains individus.

Ainsi, cette tension entre principes et pratiques, entre ce qui est valorisé et ce qui est fait instaure une ambivalence normative, entre les normes d'égalité et de féminisme qui semblent faire consensus sur le plan du discours (mais qui sont éclipsés par l'importance que revêtent les rapports de classe) et des pratiques qui se caractérisent par des rapports de pouvoir genrés. À l'UCL, les rapports de pouvoir participent au maintien de la sousreprésentation des femmes, tant sur le plan numérique que sur le plan qualitatif, en matière de prise de parole et de prise de responsabilité «à valeur ajoutée ».

Enfin, se profilent, en filigrane, certaines interprétations de l'articulation du rapport entre principes et pratiques, comme l'idée de l'impact de la socialisation différenciée d'une part, et l'effet des rapports de pouvoir d'autre part. En somme, on voit comment, dans l'idée du mouvement social sexué, les rapports de pouvoir genrés structurent l'évolution de l'organisation. 


\section{CHAPITRE 4}

\section{LES « RESSOURCES EXPLICATIVES » OU \\ LES INTERPRÉTATIONS DE LA TENSION ENTRE PRINCIPES ET PRATIQUES}

Le chapitre 3 a mis en lumière les espaces et les modalités de la contradiction/tension entre principes féministes et pratiques militantes. Les informations fournies par les militant-e-s permettent de postuler que l'UCL et, peut-être, la mouvance libertaire québécoise constituent bel et bien un monvement social sexué (Kergoat, 1992, 2001 et 2010; Bargel, 2005 ; Dunezat 1998, 1999 et 2007$)^{34}$.

Dans ce chapitre, je mettrai en lumière les « ressources explicatives » mobilisées par les interviewé-e-s pour décrire le rapport entre les principes féministes et les pratiques militantes. Autrement dit, je m'intéresserai aux explications, raisonnements et justifications mis en place pour évaluer l'écart entre les expériences quotidiennes et les principes d'égalité qui sont valorisés (Roux, 2009) par les militant-e-s, ou, du moins, qui sont mis de l'avant dans les buts et principes et la constitution de l'UCL ${ }^{35}$ et réitérés dans certains documents. ${ }^{36}$ Le chapitre 3 renvoyait surtout à la manière dont sont (re)produites les différences, tandis que ce chapitre-ci fait plutôt état du rapport à cette différence.

\footnotetext{
${ }^{34}$ Voir le point 1.3 .5

${ }^{35}$ Site web de l'UCL, Buts et principes de l'UCL http $/ /$ www causecommune.net/presentation/buts-etprincipes et Constitution http://www.causecommune.net/presentation/constitution

${ }^{36}$ Consultez, par exemple, les différentes publications de la revue Ruptures hitp://www.causecommune.net/ publications/ruptures ou bien le journal Cause Commune http://www.causecommune.net/publications/ causecommune tarchives
} 
Dans un premier temps, la notion de contradiction entre principes et pratiques sera remplacée par l'idée de la tension entre les deux, puisque les pratiques sont constamment interrogées à la lumière des principes et font l'objet d'un travail militant.

Ensuite, je discute des diverses analyses des rapports de pouvoir chez les personnes militantes: diversité de définitions, difficultés à les identifier «sur le coup» et à reconnaître leur caractère systémique. Par conséquent, plusieurs des interviewé-e-s considèrent l'UCL comme une organisation généralement égalitaire, où les rapports de pouvoir constituent des épisodes et non des processus sociaux qui jouent sur l'historique et la dynamique de l'UCL (sélection des revendications, des modes d'actions, recrutement de nouvelles personnes, etc).

\subsection{LE RAPPORT ENTRE PRINCIPES ET PRATIQUES S'EXPRIME SURTOUT EN TERMES DE «TENSION » QU'EN TERMES DE CONTRADICTION}

Au début du processus de recherche, j'avais approché l'idée de l'articulation entre principes et pratique en termes de contradiction. Certes, mes attentes de recherche ont été confirmées; les interviewé-e-s ont identifié des mécanismes (rapports de pouvoir) qui contribuent à la construction de positions inégalitaires : division sexuelle du travail militant, invisibilisation du travail des femmes, appropriation des tâches rétribuables par les hommes, sous-représentation médiatique des femmes, etc. Il m'apparaissait clair que plusieurs pratiques se situaient aux antipodes des principes d'égalité et de lutte aux oppressions qui étaient proposées dans le discours. Or, lorsque j'ai conduit les entrevues, j'ai rapidement pris conscience des limites de cette approche en termes de contradiction.

En effet, les militant-e-s identifient ces mêmes mécanismes, et en nomment (parfois) la dimension contradictoire. Toutefois, ils et elles insistent sur l'idée d'une tension entre principes et pratiques, surtout parce que pour plusieurs, l'UCL est un endroit considéré plus égalitaire que d'autres espaces d'implication (à l'exception du milieu féministe). 
Par conséquent, l'analyse sociologique doit s'attacher à rendre compte des rapports dynamiques entre les principes de luttes et les pratiques militantes, et ramener la question du rapport entre les fins et les moyens de l'action. Les réponses fournies par les militant-e-s révèlent que ni les fins, ni les moyens de l'action ne sont figés dans le temps. L'aspect informel de ce groupe libertaire permet que les pratiques soient constamment interrogées, et que soient introduites ou supprimées de nouvelles façons de faire. En fait, l'historique de l'UCL semble ponctué de "crises », où les rapports de genre, autrement latents, sont nommés. Ces crises forcent les militant-e-s à réévaluer autant les principes que les pratiques, à introduire ou modifier des mécanismes, ou, à tout le moins, à discuter de ces questions. L'absence de structure formelle favorise cette négociation constante des pratiques.

Les personnes interviewées recourent à des analyses mettant en relief cet aspect dialectique entre principes et pratiques. Ces explications concernent notamment le recours à la notion de processus et de «force de travail» à mettre en œuvre. Par ailleurs, plusieurs interventions militantes mettent en lumière la réflexion collective qui a lieu pour inventer et réinventer des façons de faire, visant à accentuer la cohérence.

Les stratégies et mécanismes ne sont pas appliqués de façon routinière : ces moyens sont vus comme le résultat de discussions collectives, et ne sont pas inscrits dans les chartes formelles, mais représentent plutôt le résultat d'un ajustement constant aux questions qui émergent.
Je sais pas si c'est explicitement écrit quelque part que les femmes peuvent "câller » un caucus non mixte. Pour moi ça va de soi, et j'ose espérer que ça sera nullement discuté. Mais je sais pas si c'est écrit formellement. - Antoine

L'extrême gauche et le féminisme n'ont pas la même relation avec la question des fins et des moyens de l'action : pour l'extrême gauche (dans la tradition anarchiste et communiste), la fin a longtemps justifié les moyens. Le mouvement féministe de la deuxième vague a prêté davantage d'attention aux processus sociaux mis en œuvre dans la lutte contre le 
sexisme et les oppressions liées au genre (Lamoureux, 2003). C'est d'ailleurs sous l'influence des pratiques horizontales du mouvement féministe, en réaction avec les pratiques excluantes des mouvements communistes, socialistes et anarchistes, que les pratiques libertaires contemporaines ont introduit des formes organisationnelles qui relèvent de la volonté de rompre avec l'attente du «grand soir »: horizontalité (volonté d'égalité, éradication des hiérarchies internes), décentralisation (autotomie des collectifs), mode d'organisation en réseau (Lamoureux, 2003).

\subsection{LES DIVERSES DÉFINITIONS DES RAPPORTS DE POUVOIR ET LEURS IMPACTS SUR L'ÉVALUATION DU RAPPORT ENTRE PRINCIPES ET PRATIQUES}

D'une part, la tension entre les principes égalitaires/antisexistes de l'UCL et la pratique militante est alimentée par le fait que.les militant-e-s proposent une variété de définitions de ce que représente, pour eux et elles, un rapport de pouvoir.

Par conséquent, plusieurs rapports de pouvoir (tels que je les ai définis dans le cadre théorique) sont invisibilisés. Plusieurs des contradictions nommées au chapitre 3 ne sont pas interprétées comme relevant de rapports de pouvoir. Ceci pourrait expliquer que de nombreuses personnes militantes rencontrées décrivent l'UCL comme une organisation égalitaire.

D'autre part, dû au caractère assez imprécis et parfois subtil des rapports de pouvoir (dû à leur caractère systémique et systématique), les militant-e-s avouent qu'il leur est difficile de les repérer. À cet effet, plusieurs soulignent la contribution des caucus non mixtes dans l'identification des rapports de pouvoir et la constitution d'un rapport de force. En dernier lieu, je mettrai en relief l'idée répandue chez les militant-e-s que l'UCL est une organisation égalitaire, ce qui n'est pas sans lien avec le fait que beaucoup considèrent les rapports de pouvoir comme des actes d'agressivité ou d'autorité coercitive.

Les tensions que j'ai identifiées au chapitre 3 relèvent de rapports de pouvoir basés sur le 
genre et renvoient indubitablement à des rapports sociaux entre groupes de sexe, c'est-àdire à des rapports antagonistes constitués autour d'enjeux (Kergoat, 2010b). Ces rapports sociaux sont d'ailleurs antérieurs à la relation sociale (ils la déterminent en quelque sorte), mais aussi postérieurs (sont activés et perpétués par l'interaction sociale) (Kergoat, 2010b), révélant de ce fait les aspects à fois structurels et relationnels de la reproduction des rapports de genre.

Or, les personnes militantes rencontrées présentent différentes façons de conceptualiser la notion de rapports de pouvoir genrés : pour certain-e-s, un rapport de pouvoir renvoie aux inégalités entre groupes sociaux (et aux mécanismes qui créent ces inégalités), tandis que pour d'autres, ils relèvent simplement du conflit interpersonnel. Je détaillerai ici ces deux positions.

D'une part, plusieurs militant-e-s réfèrent à la notion de rapport de pouvoir comme une relation découlant des inégalités entre groupes sociaux, ou comme produisant ces inégalités structurelles. Dans cette conception, les rapports de pouvoir sont définis comme la portion visible des inégalités et des oppressions sociales à plus grande échelle, ce qui se rapproche davantage des conceptions sociologiques. Ces interviewé-e-s tendent à repérer davantage de situations de pouvoir au sein de leur collectif et de la fédération. Par exemple, une militante considère que le fait que les hommes se trouvent davantage en mesure d'exercer de l'influence sur le devenir du collectif relève d'un rapport de pouvoir genré. Elle nomme aussi la disparité en matière de tâches et de prise de parole. Quelques interviewé-e-s hésitent à définir les blagues sexistes comme un rapport de domination.

Ben les blagues sexistes, je sais pas si ça peut être caractéristique d'un pouvoir de domination, mais en tout cas, $y^{\prime}$ en a, quand même. Mais ils vont tout le temps dire: ah ben c'est une blague. Mais quand même, ça reste que... Pis justement, vu que c'est nos amis aussi, c'est plus difficile de voir le rapport de domination derrière, tsé vu que c'est une blague de notre ami, c'est un libertaire, c'est un pro-féministe, tsé. - Marie-Pier

Les femmes ont dénoncé les jokes sexistes, même si on le sait ben en tant qu'anar, on le sait ben chacun dans nos têtes que ça peut pas être sérieux, 
mais ça reste des trucs, de temps en temps on va faire une joke et pour les femmes c'est lourd. - Oli

D'autre part, plusieurs autres militant-e-s font référence à des situations assimilables à des rapports de pouvoir sans toutefois les associer à un rapport de pouvoir. Ces militant-e-s réduisent la notion de rapport de pouvoir à des situations d'agressivité verbale, et associe leur propre situation à des caractéristiques personnelles plutôt qu'à des circonstances sociales.

C'est phus de la gêne, c'est plus de la gêne qui fait que je m'exprime moins, qu'une quelconque oppression, je pense.- Tanya

Par exemple, un militant constate une inégalité dans l'allocation des tâches, mais ne considère pas la situation problématique puisqu'elle ne relève pas d'une décision collective où il aurait été établi que les femmes allaient désormais prendre les tâches logistiques et les hommes les tâches visibles. Cette interprétation montre bien comment une définition différente des rapports de pouvoir peut contribuer à les invibiliser :

Ça serait un problème si par exemple, on prenait la décision collectivement de faire de la bouffe, pis on disait; bon c'est les trois filles sur les 27 gars qui vont faire la bouffe, ça, ça serait plus un problème. Non, je vois pas de... même si ça m'agace, je vois pas de problème avec ça.- Hugo

Pour un autre militant, la domination implique nécessairement un aspect coercitif ou répressif, qu'il ne retrouve pas à l'UCL;

En tout cas, avec la domination pour moi y'a une part de coercition, ou de part de représailles, pis je pense pas que si tu t'opposes, si tu prends la parole pour t'opposer théoriquement, qu'elle va se faire rabrouer par cette personne-là ou se faire peinturer dans un coin. C'est ça pour moi avec la domination il doit y avoir une part de coercition. Pis moi je pense pas en aroir jamais vu pour des désaccords ou quoi que ce soit.- Antoine

Ces conceptions duales (entre l'analyse en termes de positions sociales différenciées et celle en termes de comportement oppressant) se répercutent aussi sur l'identification des situations et comportements de pouvoir à l'UCL et fait en sorte que l'organisation est décrite comme « généralement » égalitaire 


\subsection{DES DÉFIS LIÉS À L'IDENTIFICATION DES RAPPORTS DE POUVOIR DANS LA PRATIQUE}

La définition «théorique » des rapports de pouvoir a un impact manifeste sur l'observation de ceux-ci dans la pratique militante. Ainsi, certains comportements «flagrants », qui impliquent, par exemple, des personnes agressives ou violentes sont plus facilement assimilées à des rapports de pouvoir que d'autres situations, plus subtiles, ou qui renvoient à des situations globales (liées aux positions des groupes sociaux et aux mécanismes d'assignation). Par exemple, la division du travail de conversation ou la tendance sociale à la répartition inégale du pouvoir entre les hommes et les femmes représentent des situations sous-jacentes et non des «événements» ou «accidents», lesquels commandent généralement une intervention immédiate.

En effet, «Les rapports de pouvoir liés au sexe ou au genre ne sautent pas nécessairement aux yeux des militant-e-s » (Quirion, 2008). Des militant-e-s avouent trouver difficile d'identifier ces rapports, surtout ceux qui relèvent de la deuxième catégorie :

Il y a peut-être des cas où c'était peut-être borderline, mais pas assez pour intervenir. Tu sais pas trop si tu dois... tu finis par laisser passer en te disant, ah c'est borderline, ça peut être correct.- Antoine

Malgré les zones de tensions identifiées au chapitre 3, et la position critique des femmes par rapport à ces différentiels de pouvoir, elles éprouvent des difficultés à cibler des comportements au quotidien qui participeraient à la reproduction de ces positions. Elles n'assimilent pas nécessairement la question des différentiels de statuts à des rapports de pouvoir :

Ben c'est sûr, qu'il y a des rapports de pouvoir des hommes par rapport aux fernmes, mais on dirait que j'arrive pas à l'articuler d'une manière claire. On dirait que c'est comme omniprésent, mais qu'on essaie comme de le renverser, mais que tsé, je pourrais pas te dire... j'ai de la misère à l'articuler en termes de pouvoir - Eve-Marie

Or, l'identification et l'éradication des rapports de pouvoir sont cruciales chez les libertaires, étant donné leur attachement de principe à l'établissement de relations égalitaires. 
Atteindre l'égalité ici et maintenant devrait donc faire partie de la pratique politique. Or, pour ce faire, la domination doit être définie en terme de rapport de pouvoir, et être identifiée : «En théorie, donc, l'anarchisme inclut la critique de la domination masculine, mais encore faut-il la reconnaître» (Monnet, 2001: 469).

La difficile identification des rapports de pouvoir pourrait aussi être liée au refus des structures formelles; l'absence de "postes" (présidence, vice-présidence...) pourrait contribuer à l'invisibilisation desdites hiérarchies :

Thus structurelessness becomes a way of masking power, and within the women's movement is usually most strongly advocated by those who are the most powerful (whether they are conscious of their power or not). (Freeman, 1972)

\section{L'impact des caucus non mixtes sur la visibilité des rapports de pouvoir}

Les caucus non mixtes femmes jouent un rôle important dans l'augmentation de la visibilité des rapports de pouvoir genrés. Ils permettent de promouvoir une analyse globale de ces derniers, au-delà des interactions directes (couper la parole, parler plus fort, être agressif, manquer de respect). En effet, pour «voir» les rapports de pouvoir et les situations produites par eux (hiérarchisation des luttes, division sexuelle du travail militant, sousreprésentation, etc.), les femmes doivent se constituer en collectif de femmes pour régler leur atomisation et leur éparpillement individualiste, par la création d'espaces de solidarité (Kergoat, 2010).

En dépit du fait que les militantes rencontrées sont en désaccord à propos du niveau de solidarité féministe à l'UCL, les caucus non mixtes jouent néanmoins un rôle déterminant dans l'identification des enjeux de pouvoir (en matière de rapports de genre) dans l'organisation, tant pour les femmes qui sont moins familières avec la grille féministe que pour les hommes, dont la position dans les rapports sociaux leur rend moins accessible la lecture de l'oppression qu'ils contribuent à perpétrer (Delphy, 2001; Kahane, 1998; ThiersVidal, 2001). 
Après le dernier camp, quand on avait eu le caucus non mixte, y'a des exemples qui ont été donnés par les filles là bas que moi j'avais même pas remarqué. Je ne vois pas toujours ces situations tout de suite, et quand les filles nomment des choses, souvent je fais ah ben oui, y'ont raison - Tanya

Les conclusions des femmes et des hommes suite aux caucus non mixtes sont parfois fort différentes. À ce moment, les caucus femmes permettent de nommer les choses du point de vue de celles qui vivent l'oppression et de contrer l'impression d'égalité que certains hommes ressentent;

Quand on avait fait chacun non mixte et on était revenu ensemble et c'est les gars qui avaient commencé, pis eux autres c'étaient genre, «ben nous on trouve que ça va beaucoup mieux: les filles prennent do plus en plus de tâches, elles sont bien réparties». En tout cas, je me rappelle plus du reste, mais c'était tout le contraire de ce qu'on avait sur notre papier. Pis ah oui, le pire, c'est qu'un gar's a commencé par une joke sexiste, comme pour détendre l'atmosphère. Pis après, la fille qui a parlé a dit : "ben nous on est vraiment pas d'accord", pis là elle a tout déconstruit ce que le gars avait dit en expliquant, out les filles prennent beaucoup de tâches, mais quelles tâches? Et là, elle commencé à décortiquer et là à la fin, les gars ont comme été d'accord avec nous, la plupart, en tout cas. Et tu vois que là ils avaient pas du tout vu la même réalité que nous, pour eux autres, tout était correct. Gabrielle

Les militantes soulignent qu'à force de caucus, les hommes sont de plus en plus sensibilisés à la division sexuelle du travail militant et sont plus à même d'identifier ce qui constitue un rapport de pouvoir.

\section{Est-ce que tu penses que si cette discussion avait lieu aujourd'hui, leur analyse serait différente?}

Ouais, parce que ça leur a rentré dedans, là quand même. Y'ont fait: ouais, une chance qu'on en parle, une chance que vous le dites, parce qu'on l'avait pas va comme ça.- Josiane

L'analyse des interprétations militantes des caucus non mixtes révèle à la fois la tension persistante entre principes et pratiques et les progrès inachevés en matière de féminisme. Malgré des améliorations, liées aux conclusions répétées des caucus non-mixtes, tous les hommes ne présentent pas le même degré de sensibilité par rapport aux questions de rapports de genre. Tous les degrés sont présents : de ceux qui savent définir, identifier et 
dénoncer les comportements sexistes (boyswatch), ceux qui les identifient sans agir et à ceux qui, par exemple, ne perçoivent pas nécessairement de division sexuelle du travail ou autres inégalités:

Non c'est super bien réparti les tâches. C'est bien réparti, je pense-Hugo

Par contre, le simple fait d'avoir introduit les caucus non mixtes dans la pratique organisationnelle de l'UCL témoigne de l'instauration graduelle d'un rapport de force par les femmes. De plus, cela illustre une évolution des rapports de genre, même par rapport au milieu libertaire, où la réflexion collective sur les rapports de genre ne va pas nécessairement de soi :

Mais en pratique, la plupart des groupes n'ont pas d'analyse collective sur les rapports hommes-femmes, laissant donc à l'informel les discussions à ce sujet. En fait, notre étude a permis de constater que les groupes qui agissaient explicitement sur les rapports hommes-femmes étaient ceux qui étaient composés d'une majorité de femmes. Ce sont souvent ces femmes, en s'inspirant d'outils produits par des groupes de féministes radicales, qui doivent nommer, concevoir et mettre en pratique les mécanismes permettant de travailler à la déconstruction des rapports de pouvoir. (Kruzynski et coll., 2007)

En somme, les situations d'inégalité ne sont pas toujours définies par les militant-e-s comme des rapports de pouvoir, ce qui a, sans contredit, un impact sur l'identification subséquente des rapports de pouvoir dans les réunions, les actions, les congrès et autres instances. D'autres auteur-e-s (Quirion, 2008) ont aussi fait mention du fait que les rapports de pouvoir genrés sont difficiles à démasquer, même dans les groupes où les membres sont sensibilisé-e-s à la question. L'identification des rapports inégalitaires représente un défi, notamment parce que les seuils de tolérance et de sensibilité diffèrent. Par conséquent, certaines situations qui constituent pourtant des rapports de pouvoir genrés sont interprétées comme non problématiques ou comme normales, la division sexuelle du travail influençant aussi les subjectivités (Kergoat, 2010). Malgré tout, une solidarité féministe en construction participe à la prise de conscience collective des rapports de genre, malgré que les résultats soient inégaux. 


\subsection{PAR CONSÉQUENT, L'UCL EST DÉFINIE COMME ESPACE ÉGALITAIRE}

À cause de la diversité des définitions des rapports de pouvoir genrés et des défis liés à l'identification de ces derniers, plusieurs militant-e-s définissent l'UCL comme un espace égalitaire, où les rapports de pouvoir genrés sont interprétés comme des « dérapages 》.

Il a été dit plus tôt que les rapports de pouvoir constituent des oppressions érigées en systèmes et qui sont reproduites à travers la pratique militante mixte. J'ai aussi démontré que cette dimension «systémique » n'est pas toujours reconnue par les militant-e-s lorsqu'il est temps de définir et identifier les rapports de pouvoir, et ce, malgré les efforts des caucus non-mixtes.

Par' conséquent, plusieurs interviewé-e-s (surtout les hommes) interprètent les rapports de pouvoir comme des «épisodes 》, des « choses qui arrivent », des « dérapages ». Selon eux (et elles), les rapports de pouvoir n'entachent pas la dimension égalitaire de l'UCL, puisque ces rapports sont perçus comme des «événements » et non comme des manifestations des inégalités structurelles qui placent les hommes et les femmes en position différenciée. C'est pourquoi plusieurs interviewé-e-s considèrent que l'UCL demeure, en dépit des rapports de pouvoir, une organisation égalitaire. Son rôle dans la reproduction des inégalités n'est pas reconnu. La mixité, pour les hommes surtout, n'est pas conçue comme la contradiction principale entre principes féministes et pratiques militantes. Par la bande, certains nient les inégalités structurelles, pour les raisons que j'ai identifiées plus tôt : définition en termes d'agressivité, vision des rapports de pouvoir comme des événements ou des épisodes, résistance aux caucus :

Il va peut-être y avoir des cas individuels, mais ça va pas être des cas généralisés. D'après moi les rapports sont égalitaires, mais j'ai peut-être assisté à une ou deux situations ou il y avait utilisation de langage macho. Mais pour moi c'est marginal.- Antoine

En général, oui, I'UCL est égalitaire. Oui, les rapports de pouvoir, ça peut arriver. C'est quand même pas généralisé. Pierre-Luc

Les quelques fois où il y a eu des dérapages, la plupart des gens sont 
intervenus sur le fait ou s'én sont parlés, faque ... dérapage. - Raphael

Pourtant, ces interviewés se montrent très critiques de l'état des rapports de genre à d'autres moments de l'entrevue, alors qu'ils apparaissent réticents à définir leur organisation (et ses membres) comme participant à la (re)production des inégalités lorsque la question est posée directement. Pour leur part, les femmes vont parfois analyser les rapports de pouvoir comme des dérapages, mais selon elles, l'organisation peut difficilement être définie comme égalitaire.

Non, c'est pas égalitaire c'est pas ça ma vision de l'égalité. Je pense qu'on a encore beaucoup de travail avant qu'on puisse parler d'égalité. Je trouve que dans le fond on reproduit beaucoup les choses qu'on critique. Dans le fond, les gars avec lesquels on va sentir qu'il y a plus d'égalité, ça va être les gars dans la fédé qui vont avoir le moins d'influence. Faque c'est... Alors que ça devrait être le contraire, ça devrait être ent... qui ont phus d'influence. - Émilie

Comment expliquer le fait que les rapports de pouvoir ne soient pas interprétés comme altérant le caractère égalitaire de l'organisation, où, sauf exception, les femmes s'occupent surtout de tâches logistiques et les hommes de tâches visibles? Quelles interprétations supportent le fait que les rapports de pouvoir sont vus comme des choses « qui arrivent » et non comme la manifestation de l'état des rapports de genre médiatisés par des individus? Les comportements plus «flagrants » sont interprétés souvent comme des cas individuels (lié à un individu en particulier) et isolés et non comme la manifestation de la présence d'un système qui rend l'adoption de certains comportements plus probables pour les hommes et qui fait en sorte que certaines positions plus « rétribuables » sont plus susceptibles d'être occupées par ceux-ci. Les collectifs contestataires jouent un rôle, souvent non reconnu par les militants (plus reconnu par les militantes par contre), dans le soutien et la reproduction du système.

D'ailleurs, des comportements liés aux rapports de pouvoir sont parfois attribués à la personnalité des individus et à la qualité de la relation entre ces deux individus, sans nécessairement renvoyer aux groupes sociaux auxquelles ils et elles appartiennent. En 
outre, plusieurs militants ont tendance à banaliser les rapports de pouvoir en soulignant que la situation est «mieux qu'avant» ou «mieux qu'ailleurs ». D'autres font état de la force des structures sociales en expliquant que les militant-e-s reproduisent des inégalités « malgré eux ».

\subsection{LES DÉFIS LIÉS À L'INTERVENTION SUR LES RAPPORTS DE POUVOIR}

Afin de réduire la tension entre principes et pratiques, certaines actions et interventions doivent être mises en œuvre pour contrer et dénoncer les rapports de pouvoir genrés. En effet, il est faux et idéaliste de penser que la verbalisation d'une sensibilité féministe se traduise nécessairement par l'évitement des rapports patriarcaux (Blais, 2008; Dupuis-Déri, 2008; Filleule, 2008; Drapeau, 2008; Monnet, 2001). Souvent, la préoccupation à l'égard du Patriarcat demeure théorique (Drapeau, 2008). Le chapitre trois a d'ailleurs mis en lumière les zones où les principes s'écartent des pratiques.

Plusieurs militant-e-s soulignent que, sur le plan théorique, il existe un consensus sur la nécessité d'une égalité entre les hommes et les femmes, mais que c'est en matière de mise en œuvre que ça achoppe. Un militant explique que les principes ne dictent pas des conduites précises :

Surtout sur des comportements qu'on va avoir notamment dans l'organisation qui sont pas automatiques à... c'est pas en lisant la plateforme qu'on découlerait qu'on va savoir ce que c'est un monde égalitaire, après ça, comment on fait pour gérer le pounoir dans les relations humaines. - Pierre-Luc

On ne peut pas seulement conclure que la bonne intention ne suffit pas à éliminer les hiérarchies (Blais, 2008; Filleule, 2008). Les militant-e-s abordent certes cet aspect, mais l'enrichissent d'autres interprétations. La «prise de conscience » représente une étape sur le chemin de la transformation.

Pourtant, la mouvance libertaire se caractérise par l'importance qu'elle octroie aux processus dans l'engagement militant: «les pratiques sont aussi importantes que nos 
idées! »(Luck, 2008). De plus, les moyens utilisés dans la lutte politique doivent préfigurer de l'objectif recherché; c'est pour cela que les collectifs libertaires tentent de construire des modèles alternatifs d'organisation sociale. Ceci renvoie au rapport entre les fins et les moyens de l'action; la cohérence n'est pas obtenue par magie, en nommant des principes, mais à travers un travail politique qui requiert des actions et des interventions.

Je montrerai ici que les interviewé-e-s insistent sur la nécessité de mettre en œuvre une force de travail et des stratégies pour diminuer l'écart entre les fins et les moyens de l'action. Cela m'amènera, dans un premier temps à présenter les diverses stratégies de gestion des rapports de pouvoir genrés implantées à l'UCL. Par la suite, j'aborderai plus en détails les défis reliés à une stratégie centrale à la transformation des rapports de genre dans l'UCL, soit le fait d'aborder publiquement les rapports de pouvoir.

\subsubsection{L'INTERVENTION POUR ANNIHILER LES RAPPORTS DE POUVOIR GENRÉS: UNE FORCE DE TRAVAIL QUI S'INCARNE DANS DES STRATÉGIES}

Stratégie de justification ou tentative de montrer un peu d'optimisme envers l'articulation des principes et des pratiques? Ces deux éléments semblent motiver tour à tour les interventions des militant-e-s qui abordent la tension qui subsiste entre les fins et les moyens de l'action. Pour l'expliquer, les militant-e-s rencontré-e-s soulignent que l'égalité implique un travail à long terme. Bien que l'égalité ne soit pas atteinte, plusieurs considèrent que les stratégies introduites permettent de penser qu'ils et elles sont «sur la bonne voie ».

En effet, l'égalité est analysée comme un objectif à atteindre, moyennant des efforts et une force de travail. Plusieurs refusent l'idée d'une égalité qui serait déjà atteinte (mêmes s'ils définiront l'UCL comme étant égalitaire), mais acceptent l'idée d'une égalité en construction, qui progresse à travers les stratégies, les prises de conscience, les ambiguïtés et la résistance de certains aux changements du régime de genre. 
Je ferais la nuance que l'UCL essaie d'être une organisation pro-féministe, mais c'est pas une organisation égalitaire. Dans le sens où l'égalité se construit, puis elle se construit parce que y'a des individus qui veulent la construire. Mais je pense pas que l'arganisation pourrait se targuer d'etre égalitaire, qui peut dire: "nous sommes égalitaire et nous avons aboli les rapports d'oppression " Emilie

Certain-e-s considèrent que ces efforts, quoiqu'insuffisants, illustrent néanmoins le fait que des interventions ont lieu pour modifier les rapports de genre. L'égalité est perçue comme un idéal qui anime les militant-e-s à transformer les pratiques de l'organisation et, parfois, à mettre en place des stratégies personnelles (comme le disempowerment ou le boyswatch) et collectives (féminisation, alternance homme-femme, changement dans les modes d'attribution des tâches). Comme mentionné plus haut, la notion de work in progress explique l'écart entre principes et pratiques: les stratégies représentent, justement, des pratiques militantes, du travail, visant à accroître la cohérence.

Ces stratégies, établies collectivement sont implantées pour agir en tant que « règles de gestion de la mixité », ce qui permet la régulation d'une organisation sociale mixte en se substituant au libre jeu des rapports hommes-femmes. Des solutions «techniques » sont mises en place, en attendant un changement « culturel » ou «politique » qui ferait en sorte que les un-e-s et les autres agiraient de façon égalitaire, sans y être contraint-e-s par un mécanisme ou une stratégie.

Toutefois, les femmes militantes sont en général extrêmement critiques par rapport à l'effort fourni ou par rapport à l'efficacité des stratégies mises en place. Elles se réjouissent néanmoins qu'une certaine attention soit être portée à l'égalisation, ce qui n'était pas nécessairement le cas de d'autres endroits où elles ont milité.

Les principales stratégies nommées par les militant-e-s sont détaillées ci-dessous, par catégorie: la gestion des tours de parole, la répartition des tâches, les caucus non-mixtes, le disempowerment, l'empowerment et le boyswatch. 
Gestion des tours de parole:

L'alternance homme-femme est inscrite dans la culture organisationnelle de l'UCL, et est assez répandue dans le milieu libertaire québécois ainsi que dans certaines associations étudiantes. Elle s'accompagne souvent du comptage des tours de parole, censé favoriser les gens qui en sont à leur premier tour de parole;

$Y^{\prime} a$ des gens qui parlent beaucoup, $y$ vont être en fin de liste, et ceux qui on pas encore parlé, y vont parler en premier. Et puis après, on essaie aussi de prendre en compte l'aspect genré, faque là, les femmes ont toujours un avantage en quelque sorte, pour parler. Pour que ça redevienne plus égalitaire. - Francois

Les militant-e-s pratiquent à l'occasion des tours de table, où chaque personne donne son opinion sur une question. Une dimension d'empowerment est associée aux tours de table :

Faire un tour de table ça force chaque personne à s'exprimer, à s'habituer à voir que c'est pas si sorcier que ça de parler:-Oli

Stratégies pour gérer la répartition des tâches

Ces stratégies visent une meilleure répartition des tâches dites «domestiques » qui semblaient échoir principalement aux femmes, et des tâches liées au pouvoir, qui se retrouvent concentrées surtout dans les mains de certains hommes.

Dans le premier cas, les tâches domestiques peuvent soient être allouées par pige ou par la construction collective d'un horaire pour la prise en charge des tâches identifiées.

Dans le cas des tâches liées au pouvoir, la question de la pige (au hasard) des présidences et des secrétariats a été nommée à quelques reprises, tout comme le fait de proposer des tâches de représentation publique à une femme ou à une personne qui ne l'a jamais fait. Les militantes en soulignent toutefois les effets paradoxaux, comme de mettre de la pression sur des femmes qui ne se sentent pas nécessairement prêtes à prendre la parole (manque d'expérience, manque de confiance). La stratégie du mentorat leur apparaît pertinente, mais peu mise en pratique. 
Et il m'a demandé : ça te tenterait tu de faire la conférence avec moi, on pourrait travailler ensemble. Pis ça pour moi, ça fait partie du mentorat. Ça m'a aidé beaucoup à développer une confiance dans mon discours. Émilie

Certaines femmes soulignent que les encouragements de leurs camarades les poussent à assumer des tâches que, pour diverses raisons, elles n'auraient pas assumées sans cela :

Pis tsé, le Cause Commune, des gars et des filles de l'UCL m'ont encouragé à écrire dedans. Je sais pas si c'est une stratégie, mais y'a quand même une volonté.qu'on soit plus visible. C'est des initiatives individuelles qui finissent par être adoptées par le collectif. - Eve-Marie

Ainsi, il existe des moyens visant une meilleure répartition du pouvoir, mais les militant-e-s soulignent qu'ils sont moins intégrés que, par exemple, l'alternance homme-femme ou les caucus non mixtes.

Par ailleurs, les interviewé-e-s notent que l'efficacité et l'intégration des stratégies pour favoriser une meilleure répartition des tâches semblent limitées. Certes, le mentorat et les encouragements fonctionnent, mais les hommes ne travaillent pas à attribuer du pouvoir collectivement aux femmes, mais plutôt individuellement à quelques femmes «particulièrement brillantes ou méritantes » (Kergoat, 2001a).

\section{Caucus non mixtes}

En ce qui concerne les stratégies des femmes pour modifier les rapports hommes-femmes, c'est la question des caucus non mixtes qui est la plus nommée et qui suscite le plus grand nombre d'interventions. D'une part, le caucus sert à valider avec d'autres femmes leurs impressions, et de s'assurer le support des autres femmes si cette question est ramenée en mixte. Ceci met en évidence la constitution d'une solidarité féministe à l'UCL. Par ailleurs, comme je l'ai dit plus tôt, «instaurer des espaces de non mixité pour les femmes peut être une bonne solution pour éviter (ou plutôt démystifier) les rapports de domination liés au sexe et au genre dans les organisations » (Quirion, 2008).

En fait, les caucus permettent aux femmes, minoritaires, de développer une solidarité et de 
cesser d'être atomisées et dissoutes dans la fédération (Kergoat, 2010). Enfin, la pratique des caucus non mixtes et l'instauration de comités «femmes» ou «luttes féministes » constitue une étape vers une meilleure visibilité des rapports de pouvoir et des luttes féministes. Par contre, comme c'est sur les femmes que retombe le fardeau de la preuve, la militante qui agit en tant que porte-parole peut aussi être vue comme une «casseuse de party » (ASSÉ) ou une «mauvaise tête » (Trat, 2006). Par ailleurs, les femmes ont l'impression que la pugnacité des caucus non mixtes s'érode à force de répéter les mêmes conclusions.

\section{Empowerment}

L'empowerment consiste à ce que les femmes reprennent individuellement et collectivement du pouvoir. La première étape consiste à prendre conscience de l'oppression qui est vécue, et ensuite de lui attribuer une cause politique et non psychologique. De plus, l'empowerment vise à élaborer des stratégies et tactiques pour s'opposer à l'oppression, mais également pour augmenter sa propre capacité d'action (Dupuis-Déri, 2008). En fait, les premières étapes de l'empowerment semblent franchies à l'UCL, mais il reste à ce que l'UCL en tant que groupe développe des stratégies et tactiques pour résoudre les disparités en matière de prise de tâches. Selon les interviewé-e-s qui abordent la question, l'empowerment est peu pratiqué. Ils et elles considèrent que plus d'efforts devraient être faits, notamment en matière de formation et d'autoformation, pour permettre aux femmes de développer une confiance dans leur discours, de prendre plus de place, et ce, collectivement.

\section{Disempowerment}

Les stratégies qui sont associées aux hommes sont surtout des stratégies individuelles, comme de s'intéresser individuellement aux questions féministes ou de réfléchir à ses propres pratiques. Le disempowerment consiste à réduire volontairement son pouvoir personnel sur les autres, notamment sur les femmes (Dupuis-Déri, 2008). C'est le pendant de l'empowerment: si les femmes souhaitent prendre plus de place, conséquemment, il faut 
que certains réduisent leur pouvoir et leurs privilèges;

Nous aussi on a un travail à faire, mais les gars aussi avant de sauter sur la tâche, ils pourraient attendre quelques secondes, ou la proposer à une fille

- Gabrielle

Ces stratégies peuvent aussi être appliquées collectivement : certains hommes discutent de questions féministes ou de stratégies sur le forum interne de l'UCL ou discutent ensemble de stratégies à adopter pour réduire leur pouvoir. Ces pratiques relèvent toutes du disempowerment.

Boyswatch

Le boyswatch implique, pour un homme, de rompre la solidarité avec son groupe social afin de réduire le pouvoir exercé collectivement sur les femmes (Dupuis-Déri). Par exemple, il peut s'agir de dénoncer le comportement oppressif d'un autre homme, afin que la responsabilité de dénonciation n'incombe pas toujours aux femmes. Dans les entrevues, cette stratégie n'est pas celle qui a suscité le plus d'interventions. Par contre, les exemples donnés montrent que certains hommes pratiquent le boywatch: en dénonçant des blagues/commentaires sexistes, en interpellant un gars qui coupe la parole, en rappelant aux autres de féminiser, en supportant les analyses féministes quand elles sont remises en question ou en rappelant aux autres de les intégrer.

Pis d'essayer d'intégrer des stratégies entre hommes, de dire que si quelqu'un fait une blague, je vais le nommer. Mais dans un sens, c'est pas correct d'essayer de briser la solidarité masculine. D'abord, c'est quelque chose qui n'est pas facile, quand même. C'est partout présent. - Pierre-Luc

On a eu dernièrement, quelques gars, des discussions là-dessus, sur le boyswatch. Ça a mené à ce qu'il y ait un "post "sur le fortm interne ou y'avait des textes qui expliquent c'est quoi le langage de domination, et les points sur lesquels les gars il faut se tchéquer: Quand je dis qu'il y a du travail à faire, ben c'est ça, c'est commencé un peu, et je trouve ça extrêmement stimulant depuis que je suis arrivé à l'UCL y'a des choses qui étaient pas faites, et ça se construit. -Raphaël 


\subsubsection{ABORDER LES RAPPORTS DE POUVOIR GENRÉS}

Outre les défis et difficultés liés à la définition des rapports de pouvoir en tant que situation d'inégalité et à leur identification «sur le terrain », les militant-e-s justifient également le maintien de la tension entre principes et pratiques en nommant les aléas qui entourent le fait d'aborder politiquement et publiquement les rapports de pouvoir genrés dans leurs collectifs.

La possibilité d'aborder publiquement les rapports de pouvoir genrés constitue une stratégie centrale pour les militant-e-s de l'UCL. Pour quelques personnes, il s'agit de l'unique stratégie de gestion des rapports de pouvoir qu'ils et elles ont identifiée. L'intérêt pour le pouvoir et ses manifestations constitue selon elles et eux une particularité du milieu libertaire, puisque, selon leurs observations, les autres groupes issus de la gauche radicale ne réservent pas nécessairement d'espaces pour discuter de ces questions (partis de gauche, associations étudiantes, fédérations étudiantes).

Ainsi, certains collectifs de l'UCL ont introduit un point «clarification des malaises » en début de réunion. Il ne s'agit pas d'un processus strictement féministe, mais cet espace est néanmoins utilisé pour aborder les rapports de pouvoir genrés. Par contre, les femmes soulignent que ce point est souvent bâclé, pris à la légère ou encore tourné en dérision. Les caucus non mixtes, tenus à la demande des femmes (s'ils n'ont pas été prévus à l'horaire), sont habituellement suivis d'un retour en groupe où les femmes rapportent leurs conclusions. Là encore, bien que le simple fait que cet espace soit prévu et accepté représente une ouverture en matière de rapports de genre, les femmes (et certains hommes) soulignent que les caucus ne produisent pas toujours les effets escomptés.

Comme je l'ai souligné plus tôt, les rapports de pouvoir ne font pas l'objet d'une définition consensuelle et ils sont souvent difficiles à identifier, notamment lorsqu'on parle des différences de positions produites par les rapports de pouvoir (sociaux ou issus du régime de genre du groupe). Enfin, en plus de ces obstacles, l'écart entre principes et pratiques 
pourrait être maintenu, à cause des difficultés qui entourent le fait de dénoncer publiquement et politiquement les rapports de pouvoir. D'ailleurs, plusieurs interviewé-e-s font écho aux libertaires rencontré-e-s par Quirion (2008), qui affirment qu'il leur est difficile de nommer certains problèmes liés au Patriarcat. J'exposerai ici certaines de ces difficultés.

D'une part, les rapports de pouvoir genrés sont généralement abordés hors de la vie régulière et routinière de l'organisation : outre le point "clarification des malaises » (qui semble sous-utilisé), les différentiels de position dans l'organisation et autres rapports de pouvoir sont surtout abordés dans les moments extra-ordinaires, par exemple, lorsque les femmes demandent un caucus non mixte, ou lorsque l'une d'elles soulève une question spontanément.

La lecture duale des rapports de pouvoir (agressivité versus différentiel de pouvoir) se répercute aussi sur la manière de les aborder. En effet, les comportements macho, virilistes, irrespectueux ou agressifs, bref, ceux qui émanent d'un comportement immédiat d'une personne identifiable sur le moment, semblent plus souvent faire l'objet d'une intervention immédiate. Ainsi, couper la parole, répondre de façon agressive, «monopoliser le crachoir » est plus facilement abordé que les situations d'inégalités plus générales entre les hommes et les femmes de la fédération:

Quand il y a quelqu'un qui féminise pas et qu'il se fait taper sur les doigts par un autre gars. Quand on se fait couper la parole... et qu'un autre le dénonce, et ça pour moi c'est noweau, parce que quand j'étais à la NEFAC je me faisais tout le temps couper la parole, pis là tsé comme la solution c'était de gueuler plus fort, c'était pas de....avant il fallait parler juste plus fort si tu voulais te faire entendre. - Gabrielle

Toutefois, il est moins évident et moins courant d'aborder les différentiels de situations entre les hommes et les femmes (division sexuelle du travail militant, sous-représentation, éviction de la prise de parole, hiérarchisation des luttes, sexisme en général). Ces questions sont généralement abordées hors de la vie routinière des collectifs : sur le forum interne, 
lors des assemblées générales, mais le plus souvent, c'est à l'initiative des caucus non mixtes que ces questions seront ramenées à l'ordre du jour.

Les militantes identifient certains obstacles qui rendent difficile la dénonciation des comportements oppressifs. Les militant-e-s insistent surtout sur l'obstacle que représente la dimension affinitaire pour aborder la domination masculine.

\section{L'aspect affinitaire}

Le caractère affinitaire des collectifs de l'UCL représente, pour les militant-e-s, le principal obstacle. En effet, à force de se côtoyer dans un militantisme parfois très intense, des liens étroits d'amour, de camaraderie et de solidarité se tissent entre militant-e-s, et en vient à remplacer des amitiés extra-militantes. D'autre part, la plupart des femmes du collectif de Montréal sont en couple avec un homme du collectif. Cet aspect affinitaire est identifié comme une difficulté pour nommer les comportements oppressants :

Mais en même temps, nous on était super mal, parce qu'on savait pas comment réagir parce que c'est comme tes amis, pis on disait pis on est une couple à être en couple avec des membres, pis là on se disait, là, ouais mais dans le fond c'est nos chums, c'est nos amis, qu'est-ce qu'on fait, qu'est-ce qu'on leur dit, faque on savait pas trop comment dealer avec ça. - Gabrielle

C'est pour ça que je dis que c'est délicat, ovant l'entrevue tsé on parlait des nombreux couples, ben des fois c'est des discussions de couples, et est-ce qu'on intervient au sein du couple pour dire au gars qu'il a tort et blablabla. Est-ce que ce serait condescendant, comme de dire à la fille, ben qu'elle se défend mal ou quoi que ce soit? Et si elle se défend bien, est-ce qu'il faut l'appuyer aussi? - Raphaël

L'aspect de la domination masculine :

La plupart des militantes, ainsi que certains militants soulignent que le refus, pour certains hommes, de se reconnaître comme «dominants » dans un espace supposé égalitaire, attaché à l'horizontalité peut rendre intimidante la dénonciation publique des comportements liés au pouvoir :

Je sais que ça peut être intimidant pour elles, pis euh, franchement, je trouve ça courageux de leur part, de le faire. Parce qu'elles sont vraiment 
minoritaires. À cause de la prédominance des hommes, ça peut être difficile. En plus c'est des camarades, c'est pas comme si c'était le méchant gouvernement - Hugo

D'autre facteurs participent également à compliquer la dénonciation publique des comportements, notamment, la priorité mise sur d'autres points de l'ordre du jour, le «manque de temps » et le sentiment que les gens sont peu intéressés, alors que l'idée de vivre ici et maintenant ses idéaux anti-domination devrait normalement faire en sorte que la réduction de la tension entre principes et pratiques soit considérée comme un objectif politique.

\subsection{RÉDUIRE LA TENSION ENTRE PRINCIPES ET PRATIQUES?}

Je viens d'exposer les moyens et stratégies auxquelles les militant-e-s recourent pour réduire la tension entre principes et pratiques, en matière de rapports de pouvoir genrés. Selon les interviewé-e-s, ces méthodes de «gestion de la mixité » signifient que les membres et sympathisant-e-s de l'UCL ne restent pas inertes devant les rapports de pouvoir, contrairement à ce qu'ils et elles ont constaté dans les autres organisations de gauche. En ultime instance, quelle évaluation est faite du rapport entre principes et pratiques? Quel degré de cohérence est observé? La première catégorie d'explications, plus «optimiste », concerne le constat de l'amélioration dans le temps, qui fait que l'UCL se compare avantageusement à d'autres espaces où les pratiques génèrent plus d'inégalités. Ces interprétations sont néanmoins nuancées dans un second temps par le constat du chemin qu'il reste à parcourir.

\subsubsection{DES EXPLICATIONS RELATIVISTES}

Les militant-e-s constatent que grâce à l'introduction de nouvelles stratégies, les rapports de genre «sont mieux qu'avant » et «mieux qu'ailleurs », ce qui démontre le travail qui est fait, de sorte que «c'est pas si pire ». Ces interprétations « relativistes » nuancent l'écart qui existe entre principes et pratiques, en soulignant l'amélioration qui a cours depuis le temps de la NEFAC. 
"Mieux qu'avant»

Les militant-e-s remarquent plus d'ouverture et plus de sensibilité envers les questions féministes, et réfèrent notamment à la question d'un work in progress qui se traduit par les stratégies dont j'ai parlé plus tôt. Du chemin a effectivement été parcouru depuis le temps de la NEFAC, qui présentait une quasi non mixité masculine, des pratiques cavalières, un intérêt pour le sport, un look uniforme. Plusieurs interviewé-e-s ont décrit ces aspects comme «peu invitants pour les femmes». La NEFAC avait d'ailleurs une mauvaise réputation concernant la situation des femmes. Or, les choses semblent s'être améliorées depuis ce temps, sans doute à cause de la « force de travail » mise en cuvre.

\footnotetext{
J'ai l'impression qu'il y avait une certaine contradiction, et qu'on travaille à ce qu'il y en ait plus. Parce que j'ai comme l'impression qu'on est partis du théorique et que là on essaie de mettre ça dans la pratique. Moi je trouve que c'est ça le chemin qu'on fait. Je dis pas non plus que c'était affreux, là, c'est sûr que c'est quand même une organisation que les gens te respectent, mais j'ai l'impression qu'on est en train de devenir quelque chose pour vrai. - Gabrielle
}

D'ailleurs, cette amélioration, cette idée que «c'est mieux qu'avant » est très présente dans le discours des militants. Plusieurs constatent que les rapports de genre se sont améliorés et qu'ils continuent à être transformés sous l'effet des stratégies mises en place. Le discours des interviewé-e-s distingue une rupture, entre un " avant », du temps de la NEFAC, et un « maintenant », qui semble s'être édifié à partir du congrès de refondation et de l'inclusion du principe du «privé est politique » dans la plate-forme de l'UCL.

C'était plus les anciens de la NEFAC qui parlaient, pis y disaient qu'entre la NEFAC pis l'UCL y'avait eu un gros changement... mais non, les femmes aussi le disaient, comme mettons une fille qui a été dans les deux collectifs, elle disait que depuis qu'on était l'UCL ça c'était beaucoup amélioré et que ça se traduisait notamment par le fait qu'il y a plus de fermmes. Pis c'est vrai que dernièrenent, le membership féminin a pas mal augmenté par rapport à ce qu'était la NEFAC. Elle disait aussi qu'à l'époque, la NEFAC ça donnait une image un peu gang de gars, tsé un peu macho, toute un peu du même style, et que là l'image a un peu changé et que ça faisait probablement que plus de femmes s'impliquaient. -Marie-Pier

Pour cette raison, les nouveaux et nouvelles ont l'impression d'être arrivé-e-s à l'UCL à un 
moment charnière de sa trajectoire.

Ces explications témoignent que le régime de genre d'une organisation n'est pas totalement figé et peut être modifié, même si certains résistent au changement (Connell, 2002; Kergoat, 2001a).

Quelques militant-e-s attribuent les changements positifs au départ de certains militants plus orthodoxes, plus déterministes de classe, plus catégoriques, qui résistaient à l'introduction des analyses féministes, ou à l'augmentation du rapport de force féministe. Ces débats témoignent $d u$ 《refus de changer » de certains militants, qui préfèrent quitter l'organisation plutôt que de remettre leurs analyses en question.

«Mieux qu'ailleurs »

D'autre part, l'idée que "c'est mieux qu'ailleurs » est très répandue et semble être une justification liée à un survol des autres expériences militantes vécues en mixité. Les personnes interviewées sentent que l'UCL met de l'avant plus de stratégies pour gérer les rapports de pouvoir et qu'ils et elles ressentent une volonté de lutte aux rapports de genre plus forte qu'ailleurs.

C'est quand même le lieu dans ma vie où est-ce que les rapports hommes femmes sont les plus égalitaires [...]. Par exemple, les blagues sexistes sont normalement pas tolérées, ces trucs là.-Pierre-Luc

Je pense que si on regarde le milieu de l'UCL c'est beaucoup mieux qu'ailleurs, dans le sens qu'on travaille fort, et on a les mécanismes dont on a parlé tout à l'heure pour tenter de limiter tout ça, mais on reproduit quand même les mêmes torts et travers et la même aliénation qu'on retrouve ailleurs, on est tous à un certain niveau réifié, et chaque organisation et réifiée, on peut pas y échapper. Est-ce que c'est mieux qu'ailleurs, oui.Maxime

Par contre, cette explication peut également jouer un rôle de banalisation des rapports de pouvoir. Surtout pour les hommes militants, il existe une impression que «ce n'est pas si pire que ça $»$ :

Après leur caucus non mixte, je pense que leur conclusion c'était «on est pas si pire que ça». Ben c'est vrai qu'on est pas si pire que ça, mais y'a quand même 
encore des trucs. - Marie-Pier

Ainsi, ces interprétations démontrent que les mêmes rapports de pouvoir que dans les organisations militantes en général ont cours à l'UCL, mais avec un degré moindre.

Sauf exception, les personnes militant-e-s rencontré-e-s proposent rarement ce type d'explications sans offrir de nuances. Elles et ils évaluent l'efficacité relative des stratégies et concluent presque toutes et tous à la nécessité de fournir plus d'efforts pour réduire la tension entre principes et pratiques. En effet, les militant-e-s interviewé-e-s vont, du même souffle, souligner les progrès accomplis, faire ressortir les efforts qui sont déployés pour assurer une meilleure cohérence entre principes et pratiques, avant de souligner les limites des transformations. Ceci démontre encore que l'idée de la tension convient davantage que l'idée de la contradiction.

\subsubsection{DES AMÉLIORATIONS À APPROFONDIR}

Ainsi, malgré les stratégies et les efforts, la tension persiste. J'illustrerai ici les raisons proposées par les militant-e-s. Plusieurs femmes militantes sentent que les hommes banalisent, ridiculisent ou accordent peu d'importance aux stratégies. Bien qu'en général, les hommes ne remettent pas en question les stratégies, font preuve de disempowerment et s'ouvrent au féminisme en démontrant un intérêt, d'autres résistent aux stratégies et semblent refuser de réduire leur pouvoir par rapport aux femmes. De plus, les stratégies ne sont pas toujours prises au sérieux et sont parfois ridiculisées, surtout si on pense aux caucus non mixtes. En outre, plusieurs soulignent que si les caucus permettent de nommer les inégalités et les rapports de pouvoir, peu de solutions sont trouvées ou mises en place.

\section{Ridiculiser, banaliser les stratégies}

Plusieurs femmes et certains hommes considèrent que l'écart entre principes et pratiques est maintenu, voire encouragé par les réactions aux stratégies existantes. En effet, ces personnes dénoncent le fait que certains hommes ridiculisent et banalisent les stratégies existantes, ce qui en limite l'efficacité. Ce sont surtout les caucus non mixtes et la 
« clarification des malaises » qui font l'objet de ces attitudes.

D'une part, les femmes ressentent que les hommes acceptent les caucus non mixtes, mais qu'ils n'en ont pas encore totalement intégré le sens, la nécessité et la pertinence.

Je pense que ce sont des affaires qui sont acceptées. Mais je suis pas certaine que ce soit tout à fait intégré. Y'en a des gars qui vont penser, au fait de le prévoir, dans l'horaire, mais c'est pas tous les gars. Et si on pense au demier camp de formation, ben les gars y s'étaient pas rétinis en non mixte. En général, les stratégies, c'est comme oui-oui on va le faire, mais de pas prendre le temps pour le faire. - Carolane

Par exemple, le caucus non mixte du camp de formation à l'automne 2010 a fait l'objet de nombreux commentaires. Aucun caucus non mixte n'étant prévu à l'horaire, les femmes ont spontanément décidé d'en tenir un en début de soirée. Elles ont ramené les conclusions en grand groupe le lendemain matin, tout en déplorant le fait que les hommes n'aient pas fait leur propre caucus et aient plutôt choisi de se divertir.

Y'a eu des discussions... ben pas un retour formel sur le caucus non mixte du camp de formation, mais je sais que les gars en ont reparlé. Après, l'issue de ces conversations-là, faudrait voir avec les gens qui $y$ ont participé, $y^{\prime}$ a des gens qui saluaient l'initiative pis d'autres qui trouvaient que c'était moralisateur de la part des filles d'exiger que les gars fassent des caucus non mixtes aussi. - Eve-Marie

Les femmes ont également dénoncé les blagues dont ce caucus a été l'objet;

Ah oui, ça je pense que c'est vraiment de nous ridiculiser, pis de nous... de nous ... de banaliser vraiment. "Bon les filles s'en vont parler entre elles...» «elles vont faire la liste des gars les plus macho, et elles vont faire la listes des gars les plus beaux en même temps». Genre des trucs vraiment ridicules. J'avais jamais pensé à ça, mais les gars vont vraiment essayer de ... en tout cas, y'ont vraiment une autre vision. Ils vont pas dire «ah, vous allez parler entre femmes, on comprend parce que vous vivez une oppression spécifique blablabla». Ils vont vraiment plus ridiculiser. Gabrielle

Les militantes n'ont pas l'impression que les hommes prennent leurs propres caucus au sérieux;

C'est drôle parce que quand on était dans le caucus de notre AG annuelle, 
on avait vraiment des super bonnes discussions, pis là le temps se termine faque on s'en va voir les gars, pis honnêtement, y'étaient vraiment tous en train de faire n'importe quoi, genre de se toumer les pouces et de checker le mur; et on voyait vraiment qu'ils prenaient pas ça au sérieux autant que nous, ils nous prennent pas au sérieux quand on fait ça, et ils prennent pas au sérieux les trucs non mixtes. Marie-Pier

Le point «clarification des malaises » qui peut servir à aborder des questions de pouvoir ne semble pas atteindre tout à fait ses objectifs, en raison d'une certaine incompréhension de la stratégie, mais aussi parce qu'elle n'est pas prise au sérieux.

Pis là on fait le point malaise, mais y'est pris en joke. Des fois on le prend au sérieux, pis après ça on se met à faire des jokes.[...] On a de la difficulté à appliquer sérieusement ces choses-là-Gabrielle

Cette réaction ne semble pas être unique dans le milieu militant. Des documents de l'ASSÉ font état de réactions similaires, et rapportent que les hommes font souvent porter aux féministes le fardeau de la preuve, montrant un certain manque d'ouverture et d'écoute. Beaucoup entrent dans un mode défensif, refusent de se critiquer, caricaturent des propos féministes ou les accusent de mauvaise foi (ASSÉ, 2004).

Ces réactions ont été nommées par toutes les femmes et certains hommes rencontré-e-s. Par contre, bien qu'il ne semble pas s'agir de comportements généralisés, les personnes qui mettent de l'avant ces comportements sans être dénoncées contribuent au maintien de la tension et de la contradiction.

\section{Manque de stratégies, de solutions}

Plusieurs déplorent le manque de stratégies et de solutions concrètes à apporter aux questions qui touchent l'écart entre les principes et pratiques en matière de rapports de genre. Comme je l'ai dit plus tôt, le fait de défendre certains principes ne garantit pas que les comportements iront automatiquement de pair. Par conséquemment, une force de travail doit se mettre à l'œuvre pour que changent les choses.

S'il s'avère qu'aborder et nommer les questions de pouvoir représentent une étape sur le 
chemin de la transformation, plusieurs soulignent que plus d'efforts doivent être faits. Par contre, ils et elles peinent à identifier de nouveaux moyens pour engager une véritable transformation du régime de genre de l'organisation, c'est-à-dire une véritable modification des paramètres organisationnels de l'UCL : qui est recruté-e, qui reste, qui fait quoi, quelles divisions sociales sont reconnues (Connell, 2002).

Je pense qu'on a un problème au niveau des solutions, parce qu'on nomme plein d'affaires, mais on sait pas quoi faire après. Par rapport aux tâches, par exemple, on l'a nommé aussi, mais concrètement, qu'est-ce qu'on fait? On n'a pas établi une façon de fonctionner; on se l'est juste dit. Faque c'est ça, je pense qu'on est rendu peut-être penser à des moyens, parce qu'on a peut-être la pensée magique de dire: on va le nommer ça va changer. Gabrielle

$J^{\prime} e n$ ai déjà parlé avec quelques personnes, mais c'était plus un constat, pis y'avait pas de stratégies sinon y aller avec des interventions... pis je trouve que le fait que le comité femmes justement à ce niveau-là, je dis qu'il pourrait apporter différents outils pour.. Mais même moi je compte le faire, là, à moyen terme, mais faire des outils, là, de gars.- Raphaël

Cette interprétation du manque de stratégie, de la nécessité de mettre en place plus d'efforts, de fournir un plus grand travail est plus répandue chez les femmes militantes. Un militant identifie l'un des écueils de l'amélioration de la cohérence entre principes et pratiques. Cela renvoie une fois de plus à la dichotomie entre «comportements » et «situations », que j'ai exposée en début de chapitre. En effet, si les comportements autoritaires sont plus faciles à évincer étant donné leur immédiateté, les questions d'inégalité demandent un travail de plus longue haleine :

C'est comme entre les deux, que c'est un travail à long terme et que justement parce que c'est un travail à long terme, on a tendance à la remettre à plus tard, à la faire passer après des choses qui se passent et qui sont plus importantes. Je sais pas ce serait quoi le bon mot... sur la bonne voie, mais insuffisant.- Oli

Par ailleurs, en ce qui concerne les stratégies et mécanismes mis en place pour gérer et transformer les rapports de pouvoir, une militante note que de réels efforts doivent être mis de l'avant pour que la transformation se fasse. Par conséquent, lorsqu'une solution est proposée, toutes les personnes militantes doivent foumir l'effort et la «force de travail» 
pour instaurer le changement. En l'absence d'une volonté et d'un effort de mise en œuvre, la stratégie demeure lettre morte et sans effet;

Tu mets un nouveau mécanisme, mais c'est pas dij jour au lendemain que ça va changer les choses, je pense que c'est avec la pratique du mécanisme. C'est pour ça que je dis que ça prend du temps: mais le temps, pas juste s'asseoir et regarder le temps passer, là (rires). On adopte des trucs, mais après il faut les faire. C'est là que ça bogue: on les nomme, on les a nommés plein de fois.- Gabrielle.

Impression du peu d'effet des stratégies

D'autres raisons sont invoquées par les militant-e-s pour expliquer le maintien de la tension.

En effet, plusieurs soulignent que les stratégies mises en œuvre n'ont pas l'effet désiré, et ce pour plusieurs raisons. D'une part, les militantes jugent que les caucus perdent leur efficacité et de leur pugnacité sous l'effet de la « désensibilisation » des militants.

L'effet de répétition dont j'ai fait mention plus tôt, en vertu duquel les femmes ont l'impression de répéter les mêmes conclusions, caucus après caucus, laisse supposer que les comportements identifés lors des caucus n'ont pas changé. Il s'ensuit un cercle vicieux; comme les comportements ne changent pas, les conclusions des caucus restent les mêmes, les hommes connaissent les conclusions des caucus d'avance, et l'effet de surprise et de prise de conscience s'émousse.

C'est tout le temps les mêmes choses qu'on dit. Pis là après les gars sont éccuturés d'entendre les mêmes points. Mais si c'est encore les mêmes points, ben c'est parce que ça change pas, tsé. Pis là j'étais comme, ben là c'est parce que penses-tu que ça me fait plaisir moi de répéter la même affaire?Gabrielle

En effet, les militantes supposent que les hommes agissent sous la pression du rapport de force, ce qui fait qu'ils identifient les situations de pouvoir, mais ne transforment pas nécessairement leurs pratiques dans le sens correspondant.

J'ai l'impression que les gars se sentent un peu obligés de faire comme s'ils l'avaient remarqué. Je sais pas si les hommes vont soulever des affaires, sans vraiment y croire, mais juste pour dire: " on s'en est rendu compte». Peut-être qu'ils l'ont remarqué aussi, sont pas caves, ils le remarquent, mais 
après ils font pas grand-chose et ça, les femmes elles remarquent aussi. Marie-Pier

Cette dynamique - conscience sans passage à action - est associée à plusieurs stratégies. En général, les militants sont conscients des diverses inégalités de positions à l'UCL et savent qu'une politique de féminisation a été adoptée, qu'il y a des tours de parole à respecter, mais plusieurs «oublient» de les mettre en pratique. Comme je l'ai dit plus tôt, ces stratégies sont acceptées, mais pas pleinement intégrées, au sens où elles seraient mises en œuvre. Les femmes ne comprennent pas pourquoi certains militants résistent autant à des stratégies pourtant simples:

Alors si les gars féminisent même pas leurs textes, tsé c'est comme on part de loin. Y'avait aussi des gars sur le comité qui ont contribué à féminiser les textes et tout ça. Faque ça montre encore que d'une part, y'a encore plein de monde qui y pense pas, mais que d'autres part, y'a des gens qui leur rappellent. La féminisation des textes $y^{\prime}$ en a qui ont fait «ben, pourquoi mon texte est autant féminisé?». Ben, parce que tu l'as pas féminisé toi même! Pis si tu le sais, pourquoi tu l'as pas féminisé, ton texte?- Eve-Marie

Un gars, avant le caucus, a dit que de toute façon, il savait ce qu'on allait dire, c'était genre, qu'on vous coupe la parole, que vous prenez les mêmes tâches tout le temps... des affaires de même. Faque là, j'étais comme, si tu le sais, pourquoi est-ce que ça s'arrange pas, et est-ce que tu penses que ça nous fait plaisir de revenir là-dessus à toutes les réunions. - Gabrielle

Par ailleurs, plusieurs militantes sentent que les stratégies mises en place ont des effets limités, puisqu'en dehors des réunions et des activités régulières (assemblées générales, congrès, camps de formation, réunions du conseil fédéral...), les comportements ne changent pas, comme je l'ai souligné dans la section qui traite du «privé est politique ».

Certains hommes rencontrés avouent que même s'ils sont conscients et sensibles aux rapports de domination basés sur le genre, ils leur arrivent d'oublier de mettre en œuvre les stratégies, comme de pratiquer le disempowerment ou de féminiser :

En général, j'essaie de limiter mes.., mais souvent je l'oublie, et je vais quand même partir dans une longue tirade, quand c'est pas tout le temps nécessaire. Il va m'arriver de couper la parole. Je pense que, en général, si tu coupes la parole à quelqu'un, tu vas te le faire dire. - Pierre-Luc 
En conclusion, ce chapitre visait à faire ressortir les interprétations des militant-e-s des diverses contradictions soulignées au chapitre 3. D'abord, j'ai constaté que celles-ci sont plutôt interprétées en termes de tension par les interviewé-e-s.

En début de chapitre, j'ai exposé les diverses étapes de la gestion des rapports de pouvoir : définir, identifier, aborder, et agir. J'ai également souligné les défis rencontrés à chaque étape par les militant-e-s.

La coexistence de deux définitions des rapports de pouvoir, l'une en termes d'agressivité et l'autre en termes de différentiels de situation sociale limite l'identification des rapports de pouvoir qui relèvent de la deuxième catégorie. Par ailleurs, dans tous les cas, ces derniers sont difficilement identifiables sur le coup car ils «ne sautent pas aux yeux», contrairement à ceux qui relève d'une oppression plus « directe». Les caucus non mixtes ont joué, et continuent de jouer un rôle important tant dans l'élargissement des conceptions de la notion de pouvoir que dans la dénonciation publique de ces rapports. Néanmoins, les ucélien-ne-s mentionnent que l'aspect affinitaire du collectif rend plus difficile d'aborder les rapports de pouvoir. Agir sur les rapports de pouvoir pour les éradiquer comporte également un lot de défis à surmonter, puisque, malgré l'introduction d'un certain nombre de stratégies de gestion des rapports de pouvoir genrés, plusieurs soulignent les limites rencontrées dans leur mise en cuvre. En dépit de la résistance de certains, qui banalisent, ridiculisent ou « oublient » les stratégies, les ucélien-ne-s remarquent une amélioration des rapports de genre à travers le temps : un meilleur ratio femme-homme, une sensibilisation aux enjeux de pouvoir, l'édification graduelle d'une solidarité féministe et d'un rapport de force, de plus grands efforts envers l'octroi de tâches visibles aux femmes.

En somme, animé-e-s d'un idéal d'égalité, les ucélien-ne-s disent mettre en place des stratégies et une «force de travail », qui fait en sorte que les rapports de genre sont vus comme «mieux qu'ailleurs » et «mieux qu'avant», malgré tous les défís qu'il reste à surmonter. Un militant résume: « sur la bonne voie, mais insuffisant ». 


\section{CHAPITRE 5}

\section{SYNTHÈSE ET DISCUSSION}

$\mathrm{Au}$ chapitre précédent, j'ai présenté les interprétations que proposent les ucélien-ne-s en réponse à la tension persistante entre les principes et pratiques. Or, ces seules explications sont incomplètes et ne répondent pas entièrement à mon objectif initial, qui était de saisir ce qui permettait, en ultime instance, la reproduction de rapports de pouvoir genrés, en dépit des principes féministes formalisés dans le discours et les principes.

J'ai convenu que certaines explications fournies par d'autres auteur-e-s n'étudiant pas le milieu libertaire ne s'appliquaient pas à l'UCL. En guise de rappel, les interviewé-e-s de ces recherches invoquent des arguments essentialistes (référant aux différences biologiques des hommes et des femmes) ou fatalistes (concluant que «c'est toujours comme ça ») alors que parfois, la sexuation du mouvement social s'avérait carrément invisible pour les militant-e, dont le discours ne mentionne pas les inégalités produites par les rapports sociaux (Bargel et coll., 2007, Dunezat, 2007).

Par contre, à l'UCL, les militant-e-s souscrivent tous et toutes à l'approche du genre comme construit social. Ceci n'élimine pas les logiques de justification, mais donne plutôt lieu à d'autres registres de discours. L'objet de ce mémoire n'était pas de comparer le déploiement des rapports de pouvoir dans différentes organisations, mais je me permets de souligner au passage que l'adoption, par les militant-e-s, d'une analyse en termes de «genre » et non de «sexe », leur fournit une grille d'analyse permettant de " voir » les rapports de genre, ce qui explique que les femmes anarchistes peuvent sembler plus insatisfaites de leur position 
dans leur collectif respectif que des femmes qui s'impliquent dans d'autres milieux.

Par ailleurs, l'argument du «grand soir », comme quoi le renversement du capitalisme réglerait toutes les autres oppressions dites «spécifiques» (dont l'oppression des femmes) (Dorval et coll., 1978), est beaucoup moins populaire chez les libertaires « contemporain-e$s$ » que chez les groupes d'extrême gauche des années soixante, soixante-dix et quatre-vingt (Dupuis-Déri, 2009). Cette logique de justification est donc également écartée, bien que la hiérarchisation des causes au détriment de la lutte féministe demeure, et prenne différentes formes.

Les obstacles qui s'érigent sur le chemin de l'égalité sont donc autres. Quels sont-ils? Le présent chapitre fera ressortir les analyses plus «macro» du maintien de la tension entre principes et pratiques. En effet, il ne suffit pas de conclure, comme je l'ai fait au dernier chapitre, que les rapports de pouvoir sont difficiles à définir, identifier, aborder et transformer. D'autres interprétations y apparaissent en filigrane et interpellent des questions intéressantes. Je souhaite ici les mettre en évidence et les approfondir. Il s'agit donc de faire dialoguer les analyses sociales (produites par les acteurs et actrices) et les analyses sociologiques (produites par les sociologues), puisque le genre est à la fois un travail d'analyse des chercheuses et un effort d'auto-interprétation variable de la part des interviewé-e-s (Bargel et coll., 2007). En somme, les usages sociaux (des acteurs et actrices) et sociologiques (des sociologues) du genre diffèrent, mais s'influencent mutuellement à travers le jeu des interprétations Ainsi, les chercheurs et chercheuses peuvent contribuer à la construction du genre des enquêté-e-s (et vice-versa) (Bargel et coll., 2007).

J'illustrerai d'abord que les rapports de pouvoir des hommes sur les femmes sont des processus qui contribuent à maintenir les femmes en périphérie de l'action et limitent leur participation à la direction du mouvement. Ceci se situe dans l'optique du concept de «mouvement social sexué ». Ensuite, j'aborderai l'impact de la socialisation genrée des 
militant-e-s sur les comportements politiques. Enfin, je discuterai d'une piste avancée par les femmes militantes, soit que le rapport des hommes avec le féminisme pourrait contribuer au maintien de l'écart entre principes et pratiques, puisque l'expérience différente des hommes au sein du Patriarcat institue une autre lecture des rapports de pouvoir, ce qui, combiné aux privilèges dont jouissent les hommes, contribue au maintien du statu quo genré.

Dans tous les cas, je souhaite mettre en évidence le fait que plusieurs niveaux d'analyse sont en constant dialogue; la dynamique interne des organisations (comme lieu de reproduction et de maintien des hiérarchies sociales) et le niveau structurel (le genre comme structure sociale qui oriente les comportements des individus). $\mathrm{Si}$, dans mon analyse, ces aspects ont été présentés séparément pour des raisons d'intelligibilité, reste que les structures sociales et les dynamiques internes des groupes militants entretiennent des rapports dialectiques indéniables. Par ailleurs, j'exclue la personnalité des individus, puisque cela ne fournit pas d'explications intéressantes au problème en cause : « Dans la mesure où ils [les conflits] ont pour objet la façon dont une personne dans le groupe prend l'ascendant ou semble le prendre, il est tentant d'y lire un effet de personnalité : c'est la meilleure manière de n'y rien comprendre, et de n'y rien changer » (Jordan et Vercauteren, 2007). Or, certain-e-s militant-e-s semblent attaché-e-s à ce type d'explication. J'analyserai donc en dernier lieu ce qu'il en est. Je tiens à souligner que cela ne m'amène pas à écarter le niveau de l'agencéité, puisque c'est à ce niveau que se situe l'intégration et la contestation des normes.

\subsection{LES EFFETS DES RAPPORTS DE POUVOIR SUR LA (RE)PRODUCTION DES POSITIONS SOCIALES INÉGALES}

Comme je l'ai exposé, l'approche en termes de «mouvement social sexué » postule que les rapports de genre déterminent plusieurs aspects d'un mouvement social ou d'une organisation : la sous-représentation des femmes, les comportements en réunion, la division du travail, la sélection des revendications, les structures, règles et modes d'action. 
Parallèlement, l'approche en termes de positions et positionnalités considère que les inégalités sont à la foịs mécanismes et résultats, production et produit.

Du côté des ucélien-ne-s, c'est l'aspect résultat qui est principalement reconnu. En effet, les inégalités de positions entre hommes et femmes y sont fréquemment vues comme des «pouvoirs en soi », c'est-à-dire que certains et certaines «ont» le pouvoir, comme s'il s'agissait d'un attribut. Cette approche n'est certes pas fausse, s'agissant d'un constat lucide de l'état des inégalités dans le collectif. Elle est seulement incomplète. Qu'est-ce qui participe à l'édification des positions sociales inégales?

L'aspect des mécanismes d'assignation de ces positions sociales inégales est beaucoup moins abordé par les hommes interviewés. Ce sont, en fait, principalement les militantes qui soulignent l'effet des rapports de pouvoir sur leur propre position dans leur collectif et dans la fédération. Elles reconnaissent que la dynamique qui s'opère entre les représentante-s des groupes sociaux «hommes » et «femmes » (et au sein du groupe des hommes où certaines observent des hiérarchies de masculinité) participe à la (re) production des inégalités, en dépit du fait que l'UCL se veut un espace de remise en question des rapports sociaux.

Par conséquent, les militantes abordent aussi les rapports de pouvoir genrés comme mécanismes et non seulement comme résultats. Selon elles, si les femmes sont moins nombreuses à l'UCL, c'est que les rapports de pouvoir les en détournent souvent. Elles soulignent aussi que si elles s'expriment moins (en réunion, sur le forum, dans les débats), c'est que (entre autres) des pratiques discursives oppressives ont contribué à les faire taire. Les conséquences des rapports de pouvoir sur leur participation sont donc considérables. Par exemple, leur sous-représentation dans la prise de parole fait en sorte qu'elles contribuent moins au devenir de l'organisation, à la sélection des revendications et actions :

«il y a toujours un rapport conflictuel entre les luttes féministes et celles du mouvement ouvrier et démocratique, dans les pays capitalistes développés comme ailleurs. Ceci s'explique par la reproduction, à l'intérieur des 
organisations mixtes, des rapports de pouvoir entre hommes et femmes qui se traduit à la fois par des processus plus ou moins subtils d'exclusion des femmes des instances dirigeantes et par une vision du monde et des luttes où les contradictions de sexe sont relativisées, voire masquées" (Trat, $1997: 11)$.

Ces phénomènes d'exclusion, qui n'ont pas cours qu'à l'UCL ont un impact également sur le militantisme futur des femmes impliquées.

Par conséquent, malgré certaines améliorations notables en matière des rapports de genre, l'UCL demeure un mouvement à hégémonie masculine, tant sur le plan des revendications que sur le plan des pratiques. Elle reste une organisation dont les critères et les pratiques favorisent les hommes, et où la «culture militante » est « masculine $»^{37}$. Par conséquent, les hommes semblent s'attendre à ce que les femmes intègrent les codes de l'organisation (vus comme neutres). Bien qu'il y ait une franche ouverture à l'UCL envers l'introduction de certains mécanismes pour égaliser la participation, les femmes sentent néanmoins qu'elles doivent intégrer la norme masculine dominante pour accéder à la reconnaissance des pairs. Plusieurs militantes ont l'impression que l'empowerment est parfois synonyme d'incorporation de la culture militante masculine, puisque c'est ce type de leadership qui est valorisé.

Mais pour moi, il y a comme le leadership politique et le leadership organisationnel, faque à mon avis c'est deux types de leadership qui sont assez importants au nivean de notre organisation politique. Mais en même temps ce qui est plus reconnu, c'est le leadership politique.- Émilie

À titre d'exemple, les femmes qui reçoivent des «reconnaissances symboliques » ou qui sont reconnues pour leur «capital militant» sont celles qui ont le mieux intégré les codes masculins $^{38}$.

\footnotetext{
${ }^{37}$ L'héritage de la quasi non-mixité masculine ainsi que le poids de l'esthétique traditionnelle de l'extrême gauche (ancêtres barbus, le révolutionnaire au poing levé, l'étudiant soixante-huitard, etc.) favorisent un militantisme plus ancré dans la « virilité » : langage belliqueux, emportements verbaux, etc. J'aborderai cette question plus en détail au point 5.4

${ }_{38}$ L'une des militantes qui aborde cette question identifie ces éléments comme faisant partie des codes masculins : ne pas chercher l'harmonie, ne pas avoir peur de créer des conflits politiques, passer beaucoup de temps sur le forum, lire beaucoup de théorie, s'approprier des tâches, etc. Quéniart, 2002 arrive aux mêmes
} 
Ainsi, au sein de l'UCL, des rapports de pouvoir ont cours, et ces derniers contribuent à maintenir certaines personnes à l'écart, surtout des femmes, mais aussi, aux dires de ces dernières, certains hommes qui ont atteint un niveau de « disempowerment ».

Bien entendu, ces mécanismes d'assignation ne sont pas les seuls vecteurs du maintien de l'écart entre principe et pratique, mais dans l'approche du mouvement social sexué, et selon les militantes ucéliennes, ils s'avèrent extrêmement déterminants, et enrichissent l'explication la plus répandue de la duplication des structures sociales à travers la socialisation.

\subsection{LA SOCIALISATION ET LES STRUCTURES SOCIALES PATRIARCALES}

La socialisation et l'impact des structures sociales patriarcales constituent une explication supplémentaire au maintien de l'écart entre principes et pratiques. Il s'agit d'une interprétation très répandue chez les ucélien-ne-s rencontré-e-s.

Au lieu d'invoquer un ordre naturel qui régirait les rapports entre les hommes et les femmes, plusieurs interviewé-e-s insistent sur les effets puissants de la socialisation de genre et l'influence déterminante du système patriarcal. Ces facteurs meneraient à la reproduction « automatique » ou « malgré nous » de certains comportements de domination ou de soumission.

D'une part, les auteur-e-s qui s'intéressent à la gauche radicale soulignent aussi le défi que représente l'aspect hiérarchique de la société dans la quête de l'égalité. Étant donné que les structures sociales favorisent peu l'entraide, la démocratie directe et l'horizontalité (DupuisDéri, 2009 et 2010; Kruzynski et Sévigny, 2005; Luck, 2008), « les anarchistes incorporent, comme tout le monde, certains éléments structurants de la culture nationale, notamment dans sa dimension patriarcale. Eux aussi sont susceptibles d'adopter des comportements 
misogynes, ce qui les place dans une posture contradictoire par rapport à un anarchisme qui se pense porteur d'un projet d'émancipation des femmes » (Dupuis-Déri, 2009).

Par conséquent, même si les individus possèdent une capacité à évaluer rationnellement les données du monde, et à se distancier de leur trajectoire de genre, des contraintes structurelles contraignent ces possibilités d'émancipation (Connell, 2002; Risman, 2004). Sur le plan organisationnel, les militant-e-s sont convaincu-e-s que leur forme d'organisation (le collectif), et que leur projet politique (le communisme libertaire) assure l'égalité et constitue une source d'émancipation, "alors qu'elle ne remet pas fondamentalement en cause les phénomènes d'auto-exclusion des femmes et ne permet donc pas nécessairement à chacune d'entre elles d'oser intervenir dans les débats dominés par les hommes " (Luck, 2008; 587). Si les rapports de pouvoir jouent certes un rôle dans l'écart entre les principes et les pratiques, la socialisation intervient également pour orienter les individus et rétribuer certains comportements qui sont conformes aux stéréotypes sociaux et aux définitions de ce qui constitue la féminité et la masculinité.

Les militantes reconnaissent que le monde est organisé autour de la division sexuelle du travail, à l'extérieur de l'organisation, et que cela exerce également un impact sur la division sexuelle du travail militant dans les collectifs (Luck, 2008) : «Women's participation in broad-focused social movements (e.g., civil rights, general environmental, antiwar, etc.) has often reflected society's gendered division of labor » (Culley et Holly, 2003).

D'autre part, le Patriarcat se reproduit à travers la socialisation des individus. Les ucélienne-s attribuent les différences de positions à la socialisation, à l'apprentissage de normes de comportements «masculin » et «féminin » qui se répercutent dans l'UCL, parce que profondément ancrées dans les personnes.

Les femmes sont vues, par les ucéliens, comme étant peu poussées vers la prise de responsabilités dans la sphère publique, comme prenant peu de tâches, comme peu enclines 
à assumer des prises de position tranchées ou radicales. Ainsi, on retrouve, même chez certains militants libertaires, cette croyance en la relative modération politique des femmes (Contamin, 2007). Le défi des femmes en politique est souvent de vaincre la timidité, le manque de confiance en soi (Quéniart, 2002), en plus d'apprendre à passer outre les rapports de pouvoir, et de s'affirmer (dans un contexte où elles sont souvent minoritaires). Plusieurs militantes soulignent qu'elles ont dû surmonter leur sentiment d'incompétence politique et qu'elles se sentent souvent intimidées, parce que des camarades ont usé de stratégies pour rappeler leur incompétence.

Pour cette raison, plusieurs interviewées ne se sont pas senties immédiatement à leur place dans l'organisation politique (hormis dans des tâches traditionnelles). Ce phénomène contribue autant à leur sous-représentation quantitative que qualitative. Les militantes évitent certaines tâches liées au pouvoir, prétextant ne pas les aimer, ou ne pas avoir le temps. En somme, ces arguments semblent plutôt liés à leur sentiment d'incompétence politique, à la division sociale du travail et aux rapports de pouvoir. Ces trois facteurs expliquent sans doute que les militantes sentent que les tâches liées au pouvoir leur prennent beaucoup de temps :

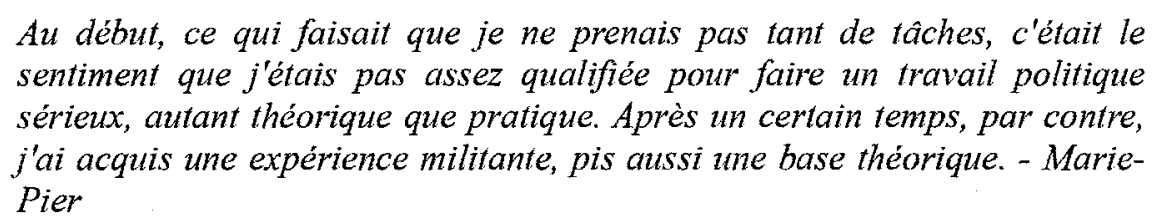

Ainsi, les attitudes politiques sont en partie déterminées par les effets de la socialisation primaire et de la socialisation politique (secondaire). Les organisations politiques jouent un rôle de socialisation secondaire: elles peuvent confirmer ou réorienter à la marge les comportements appris (Luck, 2008). C'est ce qui constitue autant le projet que le défi de l'UCL.

Or, le plus souvent, cette socialisation secondaire est genrée (Bargel, 2005) et renforce les processus de mise à l'écart des femmes à travers les rapports de pouvoir, les jeunes femmes 
étant souvent exclues des postes à responsabilité et des tâches attribuables à travers des processus qui font intervenir des mécanismes genrés (Bargel, 2005). À l'UCL, la plupart des militant-e-s ont fait leurs premières expériences dans des associations étudiantes. Elles et ils reconnaissent que ces organisations s'inscrivent dans le renforcement des socialisations genrées : la préoccupation pour le féminisme et les rapports égalitaires leur y semble moindre. Ceci laisse supposer, comme je veux le démontrer, que la problématique de la reproduction des rapports de pouvoir se joue à plusieurs niveaux : structurel (impact des normes culturelles et des divisions sociales), organisationnel (historique particulier et dynamique interne de l'UCL) et relationnel (entre représentants de groupes sociaux).

La socialisation genrée favorise les hommes pour occuper des tâches liées au pouvoir et au travail politique, étant donné que les comportements associés à la masculinité sont plus en phase avec les formes idéalisées du militantisme.

Les caractéristiques qui ont été plutôt associées aux hommes par les interviewé-e-s sont l'aptitude à la prise de parole, au leadership, la capacité à prendre leur place, entrer dans des débats, "faire le coq », adopter des prises de positions radicales. Ceci a un impact sur la mobilisation des biais masculins, c'est-à-dire la tendance des organisations dominées par des hommes à favoriser les critères et les procédures qui favorisent les hommes (Connell, 2002) . Ces attributs contribuent à faire des organisations militantes en général, et de l'UCL en particulier, des organisations sexuées.

En tant que ressource explicative, la justification de la socialisation et du Patriarcat est utilisée par les ucéliens pour expliquer leurs comportements oppressifs et leur position dominante au sein de l'UCL. Ce registre d'explication est sollicité pour atténuer l'impression de contradictions, ou pour justifier le fait qu'elles subsistent;

Les gars vont le faire aussi, ils vont le faire en joke: C'est le système, c'est pas de ma faute, j'ai été socialisé de même. Ils vont tout le temps faire ça, la joke classique, là. On est conscients et conscientes, notre analyse on est supposé le savoir, pis on sait ou s'en va, mais c'est tellement ancré en nous qu'on le fait quand même. - Marie-Pier 
Or, abolir les effets de la socialisation et déconstruire le Patriarcat implique un travail à long terme. Pour cette raison, les changements peuvent être remis à plus tard dans la pratique, au profit de problématiques plus urgentes.

C'est comme entre les deux, que c'est un travail à long terme et que justement parce que c'est un travail à long terme on a tendance à le remettre à plus tard, à la faire passer après des choses qui se passent et qui sont plus importantes. - Oli

Cette logique de justification peut être utilisée par les militants pour se déresponsabiliser en quelque sorte, et ne pas être obligé de changer eux-mêmes, ou de remettre sur le groupe dominé la responsabilité de changement (Roux, 2003). Comme je l'ai dit plus tôt, c'est souvent aux femmes de s'adapter aux codes et pratiques de l'organisation, et non l'inverse. L'empowerment est souvent vu comme un processus à sens unique, alors que le disempowerment et l'empowerment devraient être vus comme des vases communicants.

Or, si les hommes ne peuvent détruire la structure inégalitaire patriarcale ou supprimer les avantages institutionnels dont ils jouissent, ils ont néanmoins le devoir, étant donné ses principes, de s'inscrire en rupture avec l'ordre établi et de briser la solidarité masculine (Thiers-Vidal, 2001; Dupuis-Déri, 2010). Certains y mettent des efforts considérables, mais ce sont rarement les militants ayant le plus d'influence dans l'UCL. D'autre part, les hommes qui résistent le plus à la transformation des rapports de genre à l'UCL ont quitté l'organisation plutôt que d'entrer dans une remise en question critique.

Utiliser la justification de «la socialisation » pour légitimer la reproduction de certains comportements s'éloigne de l'objectif politique d'agir «ici et maintenant». Cette justification remet à un autre niveau d'intervention la lutte aux rapports de pouvoir genrés, en niant sa propre responsabilité individuelle dans le maintien et la reproduction des rapports de pouvoir genrés.

Prioriser limpact du Patriarcat et du genre en tant que structure sociale, sans invoquer la 
dynamique interne et le rôle des relations sociales dans la reproduction des rapports sociaux inégalitaires entre femmes et hommes, peut amener à discréditer les stratégies qui visent à changer les relations femmes/hommes dans l'organisation. Ainsi, les enjeux des rapports de genre sont souvent évacués parce que les militants blâment principalement leur socialisation ou le système patriarcal au lieu de se reconnaître une certaine responsabilité, pour se dédouaner de leurs comportements oppressifs (Dupuis-Déri, 2010).

\subsection{LE RAPPORT DES HOMMES AVEC LE FÉMINISME}

En filigrane des interprétations du rapport entre principes et pratiques, se profile, comme obstacle à l'abolition de la distance entre principes et pratiques, le rapport ambigu des hommes avec le féminisme et la transformation des rapports de genre. Cette question fait l'objet d'incessants débats théoriques et militants ${ }^{39}$. Je n'entends pas statuer sur la possibilité pour les hommes d'être (ou pas) féministes. Pour plusieurs raisons, dont certaines sont évoquées par les militant-e-s de l'UCL, force est de constater que le rapport des hommes au féminisme soulève des ambiguités et est sujet à débat. Cette question a été évoquée par certain-e-s militant-e-s comme obstacle à la subversion des rapports sociaux de sexe dans leur organisation.

D'une part, les militantes rencontrées constatent que les rapports de pouvoir genrés semblent moins «visibles » aux hommes, parce qu'ils ne vivent pas la même oppression, étant situé différemment dans les rapports sociaux. Ainsi, cela suppose que la différence d'expérience des hommes et des femmes au sein du Patriarcat influence nécessairement leur lecture des rapports de genre. Par conséquent, ce «poids épistémologique du vécu» pourrait représenter un facteur supplémentaire dans la reproduction des rapports de pouvoir

\footnotetext{
${ }^{39}$ Des auteurs comme Christin Delphy, dans L'ennemi principal. (2001), ou Blais (2008), Dupuis-Déri (2010), Kahane (19988) et Thiers-Vidal (2001 et 2002) émettent des réserves et soulignent les ambiguïtés du partage de la lutte féministe avec des membres du groupe social des hommes, et plaident en faveur de la pertinence du " nous femmes » et de la non-mixité organisationnelle. D'autres féministes, issues de la « troisième vague», soulèvent pour leur part les diversités de parcours des hommes et plaident pour une critique anti-essentialiste de la non-mixité et pour la construction d'un «nous féministes » plus inclusif, envers les hommes, les personnes transsexuelles ou queer (Bartky, 1998, Béchard, 2005, Mayer, 2012).
} 
genrés (Thiers-Vidal, 2002). «L'oppression est une conceptualisation possible d'une situation donnée; et cette conceptualisation ne peut provenir que d'un point de vue, c'est-àdire d'une place précise dans cette condition : celle d'opprimée » (Delphy 2002b : 281). Cette recherche a mis en lumière à plusieurs reprișes le rapport différencié des hommes et des femmes aux caucus non mixtes et la lecture différenciée qui avait été faite de la division du travail militant à l'UCL.

D'autre part, les hommes sont situés en position dominante au sein du Patriarcat, ce qui leur octroie des privilèges de classe dont ils profitent même s'ils les dénoncent. En effet, même si les hommes se disent sympathiques au féminisme, il existe un fossé entre la conviction intellectuelle et position réelle dans la division de classe (Delphy, 2001). Kahane (1998), pour sa part, suppose qu'il n'est pas impossible, quoique peu probable, qu'un homme décide de se désolidariser de son groupe social et d'abandonner volontairement les privilèges intrinsèques dont il jouit: accès probable de meilleurs revenus, sa parole sera toujours perçue comme plus crédible, pas peur de marcher seul le soir, de voyager seul, profite du rôle de protecteur, profite du travail gratuit des femmes, avoir des femmes à sa disposition pour ses plaisirs ou pour prendre soin de lui, s'attendra à inspirer le respect et l'admiration, solidarité masculine s'il s'avère antiféministe ou misogyne, moins de risque d'être harcelé ou abusé sexuellement (Dupuis-Déri, 2010).

Le décalage entre les analyses sexuées de la sexuation pourrait s'avérer un vecteur de la reproduction des rapports de pouvoir genrés, surtout si « la question du lien entre position sociale genrée et analyse des rapports sociaux de sexe n'était pas réellement posée du côté des hommes engagés et cette résistance bloque toute dynamique constructive entre féministes et hommes engagés» (Thiers-Vidal, 2002). Certains hommes à l'UCL reconnaissent leurs privilèges, mais pas nécessairement la façon dont leur position sociale limite leur capacité à identifier des situations de domination dont ils sont acteurs.

J'ai mentionné au point 5.2 que quelques militants proposent une analyse où un obstacle 
important à l'atteinte de l'égalité est «la socialisation des femmes». La responsabilité du changement de situation des groupes dominés est souvent mise dans les mains de celui-ci.

Ainsi, l'exclusion des femmes est analysée par les hommes comme étant le problème des femmes, et non pas comme le produit de la domination dont ils sont acteurs. Selon Kahane (1998), la conscience féministe chez les hommes serait en soi contradictoire puisqu'elle implique une lutte contre des avantages symboliques et matériels, qui ne leur apparaissent pas nécessairement comme des privilèges, mais comme naturels et mérités. Ces privilèges concernent tant les perspectives de vie plus avantageuses (salaire, prestige, sécurité) que la plus grande possibilité à imposer des sujets de discussion, à être considéré comme une « référence », ou la possibilité à s'identifier à des figures puissantes.

Dans nos entrevues, certains hommes identifient leur position privilégiée, mais aucun ne mentionne limpact que leur position sociale peut avoir sur leur capacité à lire les rapports de pouvoir, ou à reconnaître la sexuation du social.

Par ailleurs, les hommes n'ont objectivement, pas grand-chose à gagner du féminisme. Il est vrai que le pouvoir patriarcal bénéficie aux hommes et opprime les femmes, et alimente l'édification d'un réflexe social de domination (Dupuis-Déri, 2010). Par conséquent, certains hommes ont avantage à ce que soient maintenus intacts l'ordre social et l'état de la dynamique interne de leur organisation. Un comportement féministe implique la double nécessité d'adopter des comportements associés au groupe dominé et d'abandonner des privilèges, comme la visibilité associée au leadership politique et médiatique, souvent attribuée à la concentration de tâches entre les mains d'une même personne. Les femmes, en représentant le principal groupe subordonné dans les rapports de pouvoir patriarcaux, possèdent un intérêt structurel de changement et cherchent donc à résoudre la contradiction entre le présupposé d'égalité entre les hommes et les femmes, et une réalité où il existe un contrôle des hommes sur le travail (Connell, 2002). Les hommes qui sont désavantagés par les hiérarchies de masculinité peuvent bénéficier de la valorisation d'une plus grande 
égalité et d'une déconstruction des hiérarchies genrées, et partant, être plus motivés envers la mise en pratique et la promotion du disempowerment.

Lors d'une conférence, une participante a souligné que les hommes libertaires semblaient plus motivés à changer les structures qu'à se changer eux-mêmes. En ce sens, les militants interrogés semblent tout à fait en accord avec l'introduction de nouveaux mécanismes dans la pratique militante, mais semblent plus réticents (non pas sur le plan du discours, mais sur le plan de ce qui est dit à propos de leurs pratiques) à adopter des comportements de disempowerment, et à réduire leur pouvoir individuel et collectif. En somme, les militants et militantes identifient, d'une part des difficultés à surmonter pour qu'un homme acquière un comportement égalitaire avec les femmes, comme de réduire ses privilèges et revoir son histoire de vie à la lumière d'un historique d'oppression et de complice d'oppression (Dupuis-Déri, 2010; Kahane, 1998).

D'autre part, les militantes identifient une poignée de militants qui résistent au féminisme et refusent de changer. Ces derniers constituent une minorité décroissante, mais certes présente, au sein de l'UCL. Plusieurs militant-e-s plus ancien-ne-s attribuent l'évolution positive des rapports de genre depuis la NEFAC au départ de certains militants plus orthodoxes, plus déterministes de classe, plus catégoriques, qui résistaient à l'introduction des analyses féministes. Or, ces départs sont doublement révélateurs. $\mathrm{Si}$, d'une part, ils illustrent l'érosion de l'orthodoxie marxiste au sein de l'UCL, ils mettent aussi en lumière un certain «refus de changer» chez des hommes qui refusent de se remettre en question devant la critique féministe : ces hommes choisissent de quitter au lieu d'entrer dans une autocritique de leurs attitudes: manque de motivation à se remettre en question (Blais, 2008).

\subsection{UNE CULTURE MILITANTE MASCULINE ... MÊME DANS LE MILIEU LIBERTAIRE}

Plusieurs militantes évoquent l'idée d'une «culture militante masculine » qui contribuerait à 
la construction d'une hégémonie masculine sur les processus militants. Les personnes qui abordent cette question font surtout référence à la réputation de l'UCL. Il est nécessaire de prendre également en compte l'impact de la tradition anarchiste, qui, par son esthétique combattante, contribue à alimenter, encore aujourd'hui, une esthétique et une culture militante masculine. Ces deux aspects contribuent à édifier et entretenir une norme masculine de l'engagement, qui se traduit par une non-reconnaissance et une sousvalorisation du travail des femmes.

En effet, les sphères politiques ont longtemps été investies par une majorité d'hommes, ce qui a sans doute participé à l'édification de pratiques organisationnelles et conversationnelles associées à la construction masculine traditionnelle et « viriliste » qui se cache sous le couvert de la neutralité des pratiques. Ainsi, les nouvelles militantes qui s'impliquent doivent composer avec « un univers construit historiquement par et pour les hommes [où] rien n'est joué quant à l'admission des femmes dans le groupe; en d'autres termes, rien n'est joué par rapport à l'égalité » (Kergoat, 2001).

Cette mainmise sur les processus de mobilisation a comme conséquence «l'hégémonie masculine ». La dynamique interne de la NEFAC et de l'UCL s'est caractérisée par des comportements oppressifs qui se déroulaient presque sans opposition, comme le fait de couper la parole, d'être catégorique, d'adopter une certaine rigidité idéologique et d'être agressif dans la façon de défendre ses idées. Ainsi, la dynamique interne de la NEFAC a installé un régime de genre particulier, c'est-à-dire une série d'arrangements en matière de genre qui déterminent qui est recruté, les tâches de chacun-e, et quelles divisions sociales sont reconnues (Connell, 2002), ce qu'illustre la priorité stratégique et théorique octroyée aux divisions sociales de classes (économiques). Ainsi, les pratiques militantes des hommes représentent encore une «norme » à atteindre pour les groupes dominés. C'est une chose que remarquent les militantes, mais pas les militants, puisque souvent, «si le genre est considéré comme producteur de rapports de pouvoir, c'est uniquement à l'extérieur de l'organisation » (Bargel, 2007). Les hommes militants de l'UCL associent plus volontiers 
les inégalités à des phénomènes comme la socialisation et le Patriarcat, et moins à des processus qui impliquent une responsabilité de l'organisation.

Pourtant, les entrevues révèlent que l'UCL travaille à défaire cette réputation d'hégémonie masculine. Plusieurs personnes rencontrées ont invoqué certains aspects de la dynamique interne en abordant ce que plusieurs appellent l'aspect « gang de gars » de l'UCL, c'est-àdire qui met en œuvre ce que je viens de décrire au point précédent; culture militante masculine, où les femmes, minoritaires, ne pouvaient exercer de rapports de force, jusqu'à tout récemment. L'UCL traîne encore la réputation de la NEFAC, et le poids d'un passé anarchiste où la lutte de rue antifasciste était très présente, empreinte d'une esthétique de combats et d'opposition.

Et l'intérêt porté par tout le monde pratiquement à la NEFAC pour le foot, et c'était assez proche des milieux skinhead, et l'espèce de comportement que tut dois connaittre ... en tout cas, l'image qu'on se fait, un peu macho, dans les groupes skinhead et tout ça. Je pense que ça ça a joué et c'est une image qui est restée très longtemps à la NEFAC, même après que des gens soient partis.- Antoine

Ainsi, plusieurs interviewé-e-s associent la sous-représentation des femmes au peu d'intérêt que les femmes féministes portent à l'UCL, entre autres à cause de cette réputation de « gang de gars » issue de la NEFAC, mais qui affecte encore I'UCL. Cette réputation peut être liée aux aspects nommés précédemment: une quasi non-mixité masculine, des pratiques cavalières, un intérêt pour le sport, un look uniforme.

Enfin, la façon dont la NEFAC intervenait dans l'espace public a contribué a alimenter la réputation de la «masculinité conquérante », dont j'ai parlé plus tôt. Certain-e-s interviewée-s font référence à la nature et au mode du discours, ainsi qu'à certains différends politiques avec d'autres groupes, s'étant terminés par des foires d'empoigne.

Un, le discours public de la NEFAC était assez râpeux, c'était... pas violent, mais c'était vraiment des débats acrimonieux avec les autres tendances de l'anarchisme, surtout contre l'anarchisme que j'appelle pas organisé. C'était assez violent au niveau du langage, et c'était très tranché... tsé, eux, nous... assez binaire dans ce genre et je pense que ça ça a pas aidé, et c'est arrivé à 
plusieurs moments qu'il y a eu de la baston entre le monde de la NEFAC dans des bars avec du monde avec qui ils s'engueulaient. - Antoine

D'autre part, l'esthétique anarchiste met en scène des modèles qui invoquent la masculinité combattante et virile : l'ancêtre barbu, le terroriste, l'étudiant sur les barricades de Mai 68, black bloc (Dupuis-Déri, 2009). Dans l'esthétique militante et dans les représentations de la lutte, où sont les femmes? Pourtant, l'histoire compte plusieurs militantes, dont Emma Goldman, Louise Michel, Voltairine de Cleyre, en plus de toutes celles qui n'ont pas été retenues par l'Histoire.

De plus, même si les actions posées sont rarement violentes, la violence fait partie de l'image. De plus, la valorisation et la prégnance de la «culture ouvrière » peuvent limiter l'engagement ou la participation des femmes, qui sont historiquement et sociologiquement éloignées de la pratique militante des ouvriers (Luck, 2008).

Néanmoins, les militant-e-s de l'UCL font un certain effort, sur le plan graphique, pour changer cette esthétique, notamment en mettant en scène des femmes militantes, ou en sortant complètement de l'esthétique du combat et de l'imagerie traditionnelle ouvrière.

En somme, ces deux aspects (héritage de la NEFAC et esthétique militante) alimentent l'idée fort répandue que le «bon » militantisme est en fait lié à «norme masculine » de l'engagement. Les femmes ne se reconnaissent par dans la dynamique guerrière des organisations, et les pratiques qu'elles apportent du milieu féministe ne sont pas toujours reconnues comme du «travail politique ». Par exemple, certains hommes résistent à la « clarification des malaises », qu'ils considèrent comme une façon mièvre de communiquer. En outre, les militantes qui accèdent aux reconnaissances symboliques liées au leadership politique doivent intégrer le modèle politique dominant et se poser en adéquation avec ce qu'elles critiquent (Quéniart, 2002). Ainsi, la culture militante masculine contribue à limiter les aspirations au renversement des rapports de genre. 


\subsection{L'INDIVIDUALISATION DE L'ANALYSE DES RAPPORTS DE POUVOIR}

Les explications les plus répandues chez les militant-e-s concernent la duplication « automatique » des comportements comme effet de la socialisation et du Patriarcat. Les femmes proposent, de plus, une réflexion sur la production des hiérarchies et positions par les rapports de pouvoir entre les représentant-e-s des groupes sociaux de sexe.

Or, bien que cela puisse paraître contradictoire, certain-e-s militant-e-s expliquent les positions différenciées en les renvoyant à la personnalité des individu-e-s. Ainsi, chez certains militant-e-s, l'appropriation du pouvoir serait vue comme l'effet des dispositions psychologiques des un-e-s, par exemple: l'absence de timidité ou certaines positions professionnelles.

Ceci est une piste d'analyse secondaire, mais intéressante, en ce sens qu'elle interroge la vision que ces militant-e-s ont de leur propre organisation. Elle illustre le fait que certains militants (surtout les hommes) hésitent à reconnaître le rôle de l'UCL tant dans la subversion que dans la reproduction des rapports de pouvoir genrés. Selon ces derniers, la fédération ne modifie pas les individus et leurs rapports sociaux: les rapports entre individus et groupes sociaux seraient tributaires de mécanismes se situant au-delà (les structures sociales), et en deçà (la personnalité des individus), de l'UCL.

Dans cette optique, les individus qui sont timides restent timides, ceux et celles qui prennent la parole avaient déjà une confiance inscrite dans leur personnalité. Or, psychologiser les «désirs de prééminence " «est la meilleure manière de ne rien n'y comprendre, et de n'y rien changer » (Jordan et Vercauteren, 2008).

Or, les militant-e-s reconnaissent que les dynamiques qui déterminent l'arrangement des rapports de genre et des rapports de pouvoir sont nombreuses et fort complexes, et font intervenir de multiples dimensions: structurelles, interpersonnelles, sociales, et interindividuelles. Les militant-e-s en sont conscient-e-s et ne réfèrent généralement pas à la 
personnalité des individus sans la lier à d'autres forces et catégories d'explication.

En effet, l'UCL joue bel et bien un rôle dans la trajectoire des individus qui s'y impliquent. $\grave{A}$ travers le militantisme et les rapports qui l'entourent, il y a production, reproduction, subversion et réorganisation des rapports sociaux. C'est pourquoi toutes les stratégies et mécanismes d'égalisation sont mis en place. Produits d'une réflexion critique et collective, ces aspects permettent aux ucélien-ne-s d'accorder leur pratique à leur projet (LaurinFrenette 1981). 


\section{CONCLUSION}

Cette recherche visait à identifier les différentes analyses des militant-e-s de l'UCL en ce qui concerne l'écart entre les principes féministes de l'organisation (discours d'égalité) et les pratiques militantes. Ultimement, je souhaitais identifier les vecteurs les plus coriaces de la reproduction des rapports de pouvoir genrés, c'est-à-dire les modalités de la reproduction dans un contexte où l'idéologie dominante en la matière, c'est-à-dire l'essentialisme, le naturalisme et l'approche «culturelle » ne peuvent être invoqués pour justifier la différence. Les libertaires post-Seattle ont également évacué l'idée du « grand soir » et s'organisent afin que les fins et les moyens de l'action correspondent. Quelles analyses invoquées par les ucélien-ne-s nous informent sur les vecteurs de reproduction des rapports de pouvoir et le maintien de l'écart entre principes et pratiques?

Pour répondre à ces questions, je devais d'abord m'intéresser aux formes particulières que prend l'écart entre les principes et les pratiques. Ensuite seulement, j'ai pu dégager les justifications mises en place par les ucélien-ne-s pour expliquer le rapport entre les pratiques et principes. Ces «ressources explicatives » renvoient aux modalités de l'écart, dans la pratique. Puis, en dernière instance, j'ai voulu souligner les explications plus générales, plus « macro » que l'on pouvait dégager des récits des interviewé-e-s.

Ainsi, en premier lieu, j'ai identifié les principaux espaces où les pratiques s'écartent des principes que les interviewé-e-s associent à l'UCL. En dépit du fait que les militant-e-s se disent plus attirés par l'anticapitalisme et l'antiétatisme que par la lutte antipatriarcale, ils et elles semblent néanmoins avoir un attachement profond pour l'éradication des oppressions et des hiérarchies. Ces principes s'expriment plus précisément par le désir d'une distribution 
équitable des tâches et des prises de parole, Or, dans la pratique, les interviewé-e-s en constatent les limites : division sexuelle du travail, langue macho, et sous-représentation quantitative et qualitative des femmes. Par ailleurs, deux principes mis de l'avant dans la constitution et les buts et principes, soit «le privé est politique » et «l'anarchaféminisme », se transcrivent difficilement dans la pratique. Le premier, parce que les espaces informels (le «privé ») sont considérés par les femmes comme les lieux privilégiés de l'expression des rapports de pouvoir, qui ne sont pas soumis au même examen critique que lorsqu'ils ont lieu dans l'organisation (le "politique »). Le deuxième, parce que l'affirmation d'une articulation de toutes les sphères d'oppression (marché, État, patriarcat) se traduit néanmoins par une hiérarchisation des luttes au profit de la lutte anticapitaliste. Les militantes (surtout) considèrent que leur position dans le mouvement est le résultat des rapports de pouvoir genrés qui opèrent à l'UCL.

Avant d'expliquer ce rapport entre les principes féministes et une pratique militante qui permet la reproduction des rapports de pouvoir genrés, j'ai souligné que le rapport entre principes et pratiques s'exprimait en terme de tension plutôt qu'en terme de contradiction. En effet, les militant-e-s ne demeurent pas inertes devant les rapports de pouvoir et les zones d'achoppement de l'inégalité. Bien qu'ayant un degré de succès variable, les militante-s interrogent sans cesse les principes et leur transcription dans le réel, en introduisant, transformant ou retirant des stratégies. Ainsi, le terme de "contradiction » ne rend pas justice aux réels efforts qui sont faits et qui provoquent une impression presque unanime chez les ucélien-ne-s : celle que c'est «mieux qu'ailleurs » et que c'est « mieux qu'avant ». On ne peut toutefois pas parler de « cohérence », puisque cela occulterait les défis qu'il reste à surmonter, notamment pour faire en sorte que tous et toutes considèrent que le travail sur la dynamique interne constitue du travail politique.

Ceci dit, j'ai souligné et analysé les diverses « ressources interprétatives » mises en place par les militant-e-s pour expliquer cette tension, qui est alimentée par les rapports de pouvoir genrés. En fait, l'absence de consensus sur la notion même de rapport de pouvoir 
fait en sorte qu'il est difficile de les identifier et d'intervenir. Les militant-e-s qui définissent les rapports de pouvoir uniquement sur le plan de la relation sociale, c'est-à-dire en termes d'agressivité ou de coercition directe ont tendance à se montrer plus satisfait-e-s de l'état des rapports de genre à l'UCL, puisque selon ces personnes, ces situations se produisent plutôt rarement. Par contre, les personnes qui définissent les rapports de pouvoir en termes de mécanismes d'assignation de positions sociales remarquent d'autres défís quant à l'identification et l'intervention sur les rapports de pouvoir, notamment le fait que les pratiques oppressives sont parfois très subtiles et « ne sautent pas aux yeux ». Les caucus non mixtes semblent jouer un rôle important à l'UCL en ce qui concerne l'identification des rapports de pouvoir genrés. Par ailleurs, agir sur les rapports de pouvoir comporte certains défis. Ainsi, les diverses stratégies introduites pour rendre l'UCL plus égalitaire et « encadrer » le libre jeu des rapports de pouvoir genrés (rotation des tours de parole, empowerment, disempowerment, boyswatch, caucus non mixte) donnent des résultats mitigés, entre autres parce qu'elles constituent des solutions techniques à une question sociale. Par ailleurs, l'aspect affinitaire du collectif rend plus difficile le «bris de solidarité » que peut sembler constituer la dénonciation de comportements oppressifs. Personne n'apprécie voir ses comportements dénoncés publiquement.

Les stratégies et actions mises en place pour « gérer la mixité » donnent certains résultats, mais la tension est néanmoins maintenue. D'une part, les moments où les rapports de pouvoir sont abordés ne sont pas toujours pris au sérieux par les hommes, qui ne prévoient pas toujours un temps à l'horaire. Les stratégies sont parfois banalisées ou ridiculisées, ce qui fait que certain-e-s militant-e-s ont l'impression que les actions entreprises ont peu d'impact sur les rapports de pouvoir genrés. Par ailleurs, plusieurs soulignent qu'ils et elles se sentent à bout de ressources pour inventer de nouvelles stratégies; au-delà du constat des inégalités, peu de nouvelles pistes d'actions sont proposées.

En dépit de ce constat, les militant-e-s de l'UCL sentent qu'ils et elles sont sur la bonne voie. Ils et elles relativisent la tension entre principes et pratiques en soulignant que les 
rapports de genre à l'UCL se sont améliorés depuis le temps de la NEFAC, et que ces rapports demeurent plus égalitaires que dans d'autres sphères militantes qu'ils et elles ont expérimentées.

En dernier lieu, j'ai fait ressortir les interprétations plus larges qui se dégageaient des analyses militantes. Celles-ci expliquent, selon moi, la persistance des rapports de pouvoir genrés dans un contexte militant où l'essentialisme de genre, et l'idée que « la fin justifie les moyens" ne peuvent pas constituer des arguments. Les explications suivantes conditionnent, en quelque sorte, les comportements nommés au chapitre quatre.

L'interprétation la plus prisée concerne la socialisation (dont la socialisation politique genrée) et les structures sociales patriarcales qui orientent les femmes et les hommes vers certains comportements et aptitudes. La socialisation masculine est vue comme en adéquation avec le travail politique. Par conséquent des comportements sont reproduits en dépit de la volonté de les éradiquer. Cette explication correspond avec la vision « structuraliste » privilégiée par les anarchistes (Dupuis-Déri, 2009). Or, certains éléments à l'intérieur de l'UCL permettent une réinvention, certes inachevée, des rapports sociaux : certaines pratiques, certaines stratégies et techniques permettent de réduire les effets de la socialisation.

Les militantes rencontrées invoquent certes le rôle de la socialisation, mais insistent surtout sur les effets des rapports de pouvoir. Ceux-ci limitent la prise de parole des femmes, favorisent leur sous-représentation et entraînent une hiérarchisation des luttes au profit de la lutte anticapitaliste et un «isolement de la question femme ». Elles font intervenir le rôle des individus dans la (re)production des rapports de pouvoir genrés à l'UCL; certains militants pratiquent réellement le disempowerment, et arrivent à relativiser les effets de leur socialisation, ce qui prouve que si la socialisation genrée constitue un obstacle à l'égalité, elle ne représente pas une fatalité. 
En somme, les interprétations du maintien de la tension entre principes et pratiques sont variées et constituent autant de défis à surmonter sur la voie de l'égalité. L'UCL constitue bel et bien une organisation sexuée, où la dynamique des rapports de genre imprime le devenir du mouvement et son image publique.

$\mathrm{Au}$ terme de ce mémoire, pourrait-on affirmer que la principale contradiction entre principes féministes et pratique militante est le fait d'être organisé en mixité? La possibilité pour les hommes d'être féministe est une question qui soulève un vif débat au sein des études féministes. Certaines croient cela impossible (Delphy, 2001), d'autres en entrevoient la possibilité, mais identifient les ambiguïtés et défis spécifiques à surmonter (Kahane, 1998; Dupuis-Déri, 2009 et 2010; Thiers-Vidal, 2001), tandis que certaines jugent qu'il n'est pas approprié de considérer tous les hommes comme des vecteurs d'oppression (Bartky, 1998; Béchard, 2005). Ce clivage divise les tenant-e-s de la deuxième et la troisième vague féministe.

Or, le débat peut se déplacer vers des questions plus intéressantes. On peut notamment s'interroger sur la façon dont les hommes peuvent réfléchir leur propre rapport avec le Patriarcat. Comment, en effet, en arrivent-ils à reconnaître les privilèges dont ils bénéficient et à y renoncer? Dans tous les cas, cela implique de dépasser le poids épistémologique de leur vécu de genre. En dépit du débat théorique et politique qui pourrait fait l'objet de futures recherches, force est de constater que les militantes et les militants croient en la possibilité de s'engager ensemble sur la voie d'une égalité générale; économique, politique et entre les hommes et les femmes. 


\section{BIBLIOGRAPHIE}

ASSÉ. Document de réflexion sur le féminisme ASSÉ, 2004 [consulté le 16 mars 2012]. www.asse-solidarite.qc.ca

Alvarez, Elvita et Parini, Lorena. «Engagement politique et genre: la part du sexe. » Nouvelles questions féministes 24, no. 3 (2005), pages 106-121.

Anthias, Floya. "Rethinking social divisions: some notes towards a theoretical framework." Sociological Review 46, no. 3 (1998): pages 505-535.

Baillargeon, Normand. Les chiens ont soif: critique et propositions libertaires. Montréal: Lux Éditeur, 2001.

Bargel, Lucie. «La socialisation politique sexuée. » Nouvelles questions féministes 24 , no. 3 (2005): pages $36-49$

«Socialisation politique. » Dictionnaire des mouvements sociaux, dirigé par Olivier Fillieule. Paris: Les Presses de Science po, (2009) : pages 510-517.

Bargel, Lucie, Éric Fassin, et Stéphane Latté. «Usages sociologiques et usages sociaux du genre: le travail des interprétations. " Sociétés et Représentations, vol 2, no. 24 (2007): pages 59-77.

Bartky, Sandra. «Foreword » Men doing Feminism, dirigé par Tom Digby. New York: Routledge, (1998). pages xi-xiv.

Béchard, Marie-Josée. «La relation entre les hommes et le féminisme: une question de rapports de pouvoir.» Dialogues sur la troisième vague féministe, dirigé par Maria Nengeh Mensah. Montréal: Remue-ménage, 2005. pages 174-189. 
Bereni, Laure, Sébastien Chauvin, Alexandre Jaunait, et Anne Revillard. Introduction aux gender studies: manuel des études sur le genre. Bruxelles: Groupe de Boeck, 2008.

Blais, Mélissa, Isabelle Courcy. «Prolétaire de tous les pays, qui lave vos chaussettes? » Nouveaux Cahiers du socialisme, no. 4 (2010).

Blais, Mélissa. «Féministes radicales et hommes proféministes: l'alliance piégée » dans Québec en mouvement, dirigé par Francis Dupuis-Déri. Montréal: Lux, 2008, pages 147-175.

Breton, Émilie, Julie Grolleau, Anna Kruzynski et Catherine Saint-ArnaudBabin, «Mon/notre/leur corps est toujours un champ de bataille: discours féministes et queers libertaires au Québec, 2000-2007 ». Recherches féministes, vol. $20, \mathrm{n}^{\circ} 2$ (2007) p. 113-139.

Brouillette, Véronique, Nathalie Guay et Flavie Achard. "Portrait des luttes féministes dans les organisations mixtes." Nowveaux Cahiers du socialisme : Luttes, oppressions, rapports sociaux de sexe, no. 4 (2010): pages 219-229.

Collectif Ephesia. La place des femmes: les enjeux de l'identité et de l'égalité au regard des sciences sociales. Paris: La Découverte, 1995.

Connell, R. W. «Gender Relations.» dans Gender, édité par Polity Press. Cambridge, 2002, pages 53-70.

Daune-Richard, Anne-Marie, et Anne-Marie Devreux. «Rapports sociaux de sexe et conceptualisation sociologique » Recherches féministes, vol 5, no. 2 (1992): pages 7-30.

Delphy, Christine, 2002b «Pour un féminisme matérialisme », dans C. Delphy, L'ennemi principal, tome I : «Économie politique du patriarcat », Paris, Syllepse : 271-282.

Descarries, Francine, et Marie Mathieu. Entre le bleu et le rose: stéréotypes sexuels et construction sociale du masculin et du féminin. Québec: Conseil du statut de la femme, 2009. 
Drapeau, Marie-Hélène. «Lutte pour le droit au logement: perspective de déconstruction du pouvoir dans un espace autogéré : le squat du 920 de la Chevrotière. " Mémoire de maîtrise, Université Laval, 2007.

Dunezat, Xavier. «Des mouvements sociaux sexués. » Recherches féministes 11, no. 2 (1998): pages 161-195.

- "La division sexuelle du travail militant dans les assemblées générales: le cas des mouvements "Sans". » AMNIS: Revue de civilisation contemporaine de l'Université de Bretagne, no. 8 : Femmes et militantisme (2008).

- _La fabrication d'un mouvement social sexué; pratiques et discours de lutte. 》Sociétés et Représentations 2, no. 24 (2007): pages 269-283.

- «Le traitement du genre dans l'analyse des mouvements sociaux: France et ÉtatsUnis. " Cahiers du Genre hors-série no 6: Féminismes: recompositions et mutations, dirigé par Dominique Fougeyrollas-Schwebel. Paris: L'Harmattan, 2006. pages $117-142$

_ـ «Mouvements sociaux sexués: reproduction et changements. » Cahiers du Genre Un continent noir: le travail féminin, no. 26 (1999): pages 101-110.

- "Trajectoires militantes et rapports sociaux de sexe. » Paris: Les presses de sciencesPo, (2009). pp. 243-260

Dupuis-Déri, Francis. «Hommes anarchistes face au féminisme: pistes de réflexions au sujet de la politique, l'amour et la sexualité. » Réfractions, no. 24 (2010): pages 107-121.

. «'anarchisme face au féminisme: comparaison France-Québec. 》 Le sexe du militantisme, dirigé par Les Presses de SciencesPo. Paris, 2009. pages 187-204.

- «Les hommes proféministes: compagnons de route ou faux amis. » Recherches féministes, 21, no. 1 (2008): pages 149-169.

Falquet, Jules. «Trois questions aux mouvements sociaux «progressistes ». Apport de la théorie féministe à l'analyse des mouvements » Nouvelles questions féministes 24 , no. 3 (2005): pages $18-39$.

Filleule, Olivier. « Travail militant, action collective et rapports de genre. » Travaux de science politique - Political Science Working Paper Series Université de Lausanne, 
no 36 (2008): pages 1-61.

Filleule, Olivier, Patricia Roux, et Lilian Mathieu. «Introduction. » Politix - Militantisme et hiérarchies de genre 2, no. 78 (2007): pages 7-12.

Freeman, Jo. «The Tyranny of the Structurelessness.» Berkeley Journal of Sociology, no 17 (1972): pages 151-165.

French, Marilyn. La fascination du pouvoir. Paris: Acropole, 1986.

Foucault, Michel. "Le sujet et le pouvoir. 》 Dits et écrits 1954-1988 par Michel Foucault. Paris: Éditions Gallimard, 1982 : pages 222-243.

—. «Les mailles du pouvoir. » Dits et écrits 1954-1988 par Michel Foucault. Paris: Éditions Gallimard, 1982 : pages 183-201.

—_. "Cours du 14 janvier 1976. » "Il faut défendre la société »: cours au Collège de France. Paris: Editions Gallimard, 1976.

Gauthier, Benoît. «La structure de la preuve. » Recherche sociale: de la problématique à la collecte des données. Québec: Les presses de l'Université du Québec, 2009b. pages $169-198$

Graeber, David. «La démocratie des interstices. » Revue du MAUSS 2, no. 26 (2005): pages 41-89.

- Fragments of an Anarchist Anthropology. Chicago: Prickly Paradigm Press, 2004.

Grolleau, Émilie et Kruzynski, Anna. «Vers la construction d'un nouvel imaginaire : un exposé sur la théorie de la stratification sociale de Floya Anthias » Communication présentée dans le cadre du colloque "Différenciation sociale et épistémologie féministe », ACFAS Trois-Rivières, les 8 et 9 mai 2007.

Guillaumin, Colette. "Sexe, race et pratique du pouvoir: l'idée de nature. » Dirigé par Côtéfemmes, Paris, 1992, 239 pages. 
Gygax, Pascal, et Noelia Gesto. «Féminisation et lourdeur de texte. » L'année psychologique, no. 107 (2007): pages 233-250.

Harding, Sandra. «Can Men Be Subjects of Feminist Thought.» Dans Men Doing Feminism, dirigé par Tom Digby, New York: Routledge, 1998. pages 171-195

- The Feminist Standpoint Theory Reader: Intellectual and Political Controversies. New York: Routledge, 2004.

- «"Strong Objectivity": A Response to the New Objectivity Question.» Synthese 104, no. 3: Feminism and Science (1995): pages 331-349.

Héritier, Françoise. Masculin/féminin: dissoudre la hiérarchie. 2 vols. Vol. 2. Parod: Odile Jacob, 2002.

Hirata, Helena et coll. Dictionnaire critique du féminisme. Paris: Presses Universitaires de France, 2000.

Kahane, David. «Male Feminism as an Oxymoron.» dans Men doing feminism, dirigé par Tom Digby. New York: Routledge, 1998. pages 213-235

Kergoat, Danièle. Les infirmières et leur coordination 1988-1989. Paris: Éditions Lamarres, 1992.

- «Le syllogisme de la constitution du sujet sexué féminin: Le cas des ouvrières spécialisées. " Travailler 2, no. 6 (2001): pages 105-114.

« Le rapport social de sexe: de la reproduction des rapports sociaux à leur subversion. " Actuel Marx, no. 30 (2010a): pages 60-75.

-.. "Une sociologie à la croisée de trois mouvements sociaux.» L'homme è la société 2, no. 176-177 (2010b): pages 27-42.

Knoll, Stefanie, and Elof Aragorn. 2010 Anarchist Survey Report 2010 [page consultée le 3 
mars 2012]. http://www.anarchistsurvey.com/.

Kruzynski, Anna. «De l'opération SalAMI à Némésis; le cheminement d'un groupe de femmes du mouvement altermondialiste québécois. " Recherches féministes 17, no. 2 (2004): pages 227-262.

Kruzynski, Anne, G. Lambert-Pilotte, et Marie-Hélène Drapeau. « La révolution est possible: un portrait des groupes libertaires autogérés au Québec. » Possibles 1 (2007).

Kruzynski, Anna, et Marcel Sévigny. «Réhabiliter la politique: Une option libertaire. » Possibles 29, no. 1 (2005): pages 28-45.

Kruzynski, Anna, Rachel Sarrasin, et Émilie Breton. (proposition d'article accepté, publication prévue au printemps 2013). Une culture politique antiautoritaire au Québec (titre provisoire), Lien social et politiques (Radicalités et radicalisations la fabrication d'une nouvelle "norme" politique?). 2013.

Laurin-Frenette, Nicole. "Féminisme et anarchisme: quelques éléments théoriques et historique pour une analyse de la relation entre l'État et le mouvement des femmes " Femmes et Politique, dirigé par Yolande Cohen. Montréal: Les Éditions le jour, 1981. pages 147-191

Lamoureux, Diane. «Et si le socialisme avait à apprendre du féminisme? 》 Tribunes solidaires (2003).

Luck, Simon. «Sociologie de l'engagement libertaire dans la France contemporaine: socialisations individuelles, expériences collectives et cultures politiques alternatives. », Thèse de doctorat, Université Paris 1 Panthéon-Sorbonne, 2008.

Lukes, Steven. Power, a Radical View. Londres: MacMillan Press, 1974.

Mathieu, Nicole-Claude. L'anatomie politique: catégorisations et idéologies du sexe. Paris: Côté-Femmes, 1991.

Miles, Matthew and Michael Huberman. Analyse des données qualitatives. Bruxelles: De Boeck Université, 2003. 
Militantisme et hiérarchie de genre, (sans auteur), Politix, 2007, vol 2, no 78, pp 3-4.

Monnet, Corinne. «De l'antiféminisme chez les anarchistes. " L'anarchisme a-t-il un avenir? Histoire de femmes, d'hommes et de leurs imaginaires, dirigé par Collectif. Lyon: Ateliers de création libertaire, 2001. pages 467-473

— - "La répartition des tâches entre les femmes et les hommes dans le travail de conversation. » Nouvelles questions féministes 19 (1998): pages 9-34.

Ostrander, Susan A. «Gender and Race in a Pro-feminist, Progressive, Mixed gender, mixed race organization.» Gender \& Society 13, no. 5 (1999): 628-642.

Pilotte, Magaly. « Modalités, formes et significations de l'engagement au sein des collectifs du mouvement de justice globale: étude de cas auprès de 16 militant-e-s montréalais-e-s. » Mémoire de maitrise, Université du Québec à Montréal, 2006.

Piotte, Jean-Marc. «Foucault et les anarchistes. » Combats, vol 9, no. 3-4 (2007): 20-21.

Quirion, Marie-Ève. "Les rapports de pouvoir au sein des groupes militants radicaux.» Mémoire de maîtrise, Université du Québec à Montréal, 2008.

Roux, Patricia. «Détournements et retournements du principe d'égalité » Nouvelles questions féministes 22, no. 3 (2003): 4-11.

—. "Résistances à l'égalité entre femmes et hommes.» Recherche féministe francophone: langue identité enjeux, dirigé par Fatou Sow. Paris, 2009. 108-117.

Roux, Patricia, et coll. « Le militantisme n'échappe pas au patriarcat. » Nouvelles questions féministes 24, no. 3 (2005).

Roux, Patricia et Olivier Filleule. «Avant-propos. » Le sexe du militantisme, dirigé par Olivier Filleule and Patricia Roux. Paris: Les Presses de Science Po., 2009. 11-22

Roventa-Frusumani, Daniela. Concepts fondamentaux pour les études de genre. Paris: Éditions des archives contemporaines, 2009. 
Thiers-Vidal, Léo. «De la masculinité à l'anti-masculinité: penser les rapports sociaux de sexe à partir d'une position sociale oppressive. » Nouvelles questions féministes 21 , no. 3 (2002): $71-83$.

- « Rapports sociaux de sexe et de pouvoir: une comparaison des analyses féministes radicales avec des analyses masculines engagées. » Université de Genève, Université de Lausanne, 2001.

Trat, Josette. «Introduction. » Cahiers du GEDISST, no 18: Hommes et femmes dans le mouvement social, dirigé par Josette Trat et Jaqueline Heinen. Paris: L'Harmattan, 1997. pages $5-21$

_. «La responsable féministe, la "mauvaise tête" dans les organisations mixtes. » Cahiers du Genre 3, no. 1 (2006): 143-158.

Yin, Robert K. Case Study Research: design and methods. 2 ème édition. London: SAGE Publications, 1994. 


\section{ANNEXE 1}

\section{PROTOCOLE D'ENTREVUE}

\section{QUESTIONS BRISE-GLACE}

1.1. Tu milites dans l'UCL depuis combien de temps?

1.2. Es-tu membre ou sympathisant-e? (pourquoi l'un et pas l'autre)

1.3. Combien y-a-t-il de membre dans ton collectif? De sympathisant-e?

\section{PRINCIPES DE L'ORGANISATION}

2.1. Comment en-es tu arrivé (e) à militer dans l'UCL?

2.2. Quels aspects de l'UCL t'ont particulièrement intéressé?

2.3. Que répondrais-tu à une personne qui te demande ce qu'est l'UCL et qui ne connaît rien à ce sujet? (buts, principes, actions?)

2.4. Que dirais-tu à cette même personne si elle te demandait ce que ça veut dire d'être libertaire?

2.5. Selon toi, qu'est-ce qui fait en sorte que l'UCL ait décidé de se définir comme anarcha-féministe après sa rupture avec la NEFAC? (quels débats, prises de conscience). Es-tu en accord avec ces buts et principes?

2.6. Comment te situes-tu par rapport à la question du féminisme?

2.7. Penses-tu que la question de la lutte de classe et du féminisme sont compatibles?

2.8. Dans ton organisation, sens-tu une sensibilité à l'égard du féminisme?

\section{QUESTIONS CONCERNANT LES PRATIQUES}

Sous-représentation des femmes

3.1. Quel est le ratio homme/femme

a)dans ton collectif local?

b)Au Fédéral

3.2. Comment expliques tu le fait que les femmes soient moins nombreuses dans l'organisation?

Thématique des actions et publications

3.3. Quelles sont les dernières actions que ton Collectif a organisé ou auquel il a participé?

3.4. Quelles sont les thématiques de tes publications (si tu publies)? 
3.5. Penses-tu que les principes féministes de l'organisation sont bien représentés dans les actions et publications? En quoi?

Tâches, division du travail

3.6. Peux-tu me parler un peu de la façon dont sont attribuées les tâches dans ton collectif? (mode de prise de décision).

3.7. Quelles sont les tâches que tu assumes le plus souvent?

3.8. Y-a-t-il des tâches que tu voudrais assumer mais que tu ne fais pas? Pourquoi?

3.9. Quelles sont les tâches généralement assumées par les femmes dans ton collectif?

3.10. Quelles sont les tâches généralement assumées par les hommes dans ton collectif?

3.11. Que penses-tu de cette différence? La juges-tu souhaitable ou au contraire problématique? Comment expliques-tu que cette différence perdure malgré les principes antisexistes de l'organisation?

Leadership et prise de parole

3.12. Est-ce que certaines personnes exercent plus d'influence (leadership) que d'autres dans ton Collectif ou dans la Fédé? Quelles sont les caractéristiques de ces personnes (âge, sexe, études)?

3.13. Est-ce que certaines personnes prennent plus la parole que d'autres en réunion, dans les congrès...

3.14. Est-ce que certaines personnes sont plus écoutées quand elles parlent? Quelles sont les caractéristiques de ces personnes?

4.RAPPORT ENTRE PRINCIPES ET PRATIQUES

4.1. Qu'est-ce que ça implique pour toi d'être libertaire, dans tes relations avec les autres?

Application pratique du féminisme

4.2. Te considères-tu fémịniste (pro-féministe)?

a) Quelles actions cela implique-t-il?

b) Que fais-tu pour mettre ça en pratique?

4.3. Quelles sont les actions mises en ouvre dans l'UCL pour être cohérent avec les principes féministes qui ont été adoptés?

\section{Rapports égalitaires}

4.4. Penses-tu que les membres entretiennent des rapports égalitaires dans l'UCL?

4.5. Les rapports hommes-femmes te semblent-ils égalitaires?

4.6. Remarques tu des rapports de pouvoir? A quels niveaux?

4.7. Quelles sont les stratégies des femmes pour modifier les rapports femmes-hommes dans ton Collectif?

a) Comment réagis-tu à ces stratégies?

b) Comment les autres réagissent-ils généralement? 
4.8. Quelles sont les stratégies des hommes pour modifier les rapports femmes-hommes dans ton Collectif?

a) Que penses-tu de ces stratégies?

b)Comment les autres réagissent-ils généralement?

Articulation principes/pratiques

4.9. Lors que tu penses aux principes de l'UCL et aux pratiques dont on a parlé, as-tu en tête une impression de cohérence ou de contradiction (développper)

4.10. Que penses-tu du fait que l'UCL se présente comme une organisation anti-sexiste et anarcha-féministe, mais que tu observes en même temps une DST et des RPBG?

4.11. Par quoi est-ce causé selon toi? (socialisation? Nature humaine? Manque de volonté?) 


\section{ANNEXE II}

\section{LA LANGUE MACHO}

JOUER AU "SOLUTIONNEUR" DE PROBLEMES

Être toujours celui qui donne la réponse ou la "solution", avant que les autres n'aient eu quelque opportunité de contribuer à l'échange.

MONOPOLISER LE CRACHOIR

Parler trop souvent, trop longtemps et trop fort.

\section{PARLER EN "MAJUSCULES"}

Présenter ses opinions et ses solutions comme le point final sur tout sujet, attitude renforcée par le ton de la voix et l'attitude physique.

\section{ATTITUDE DEFENSIVE}

Répondre à toute opinion contraire à la sienne comme s'il s'agissait d'une attaque personnelle.

\section{COUPER LES CHEVEUX EN QUATRE}

Soulever chaque imperfection des interventions des autres et une exception à chaque généralité énoncée.

\section{DIRIGER LA SCENE}

Prendre continuellement la responsabilité des tâches-clé avant que les autres n'aient la chance de se porter volontaires.

\section{REFORMULER}

Reprendre en ses propres mots ce qu'une personne (le plus souvent une femme) vient de dire de façon parfaitement claire. Embarquer sur la conclusion d'une intervention pour la récupérer à ses propres fins (phénomène du "recouvrement").

\section{CHERCHER LES FEUX DE LA RAMPE}

Se servir de toutes sortes de stratagèmes, de mises en scène, pour attirer un maximum d'attention sur soi, ses idées, etc. 


\section{RABAISSER}

Commencer ses phrases avec des effets du genre : "Auparavant je croyais cela, mais maintenant..." ou "Comment peux-tu en venir à dire que..."

\section{PARLER POUR LES AUTRES}

Faire de ses opinions la voix d'une collectivité pour leur donner plus de poids : "Beaucoup d'entre nous pensons que...". Interpréter à ses fins ce que disent les autres : "Ce qu'elle veut dire, en fait, c'est que...".

\section{FAIRE DU "FORCING"}

Imposer comme seuls valables la tâche et le contenu, en éloignant le groupe de l'éducation de chacun-e, ainsi que d'une attention au processus de travail collectif et à la forme des productions.

\section{DÉPLACER LA QUESTION}

Ramener le sujet de la discussion à quelque thème que l'on maîtrise, de façon à briller en donnant libre cours à ses dadas.

\section{NÉGATIVISME}

Trouver quelque chose d'incorrect ou de problématique à tout sujet ou projet abordé.

\section{N'ÉCOUTER QUE SOI}

Formuler mentalement une réponse dès les premières phrases de la personne qui parle, ne plus écouter à partir de ce moment et prendre la parole à la première occasion.

\section{INTRANSIGEANCE ET DOGMATISME}

Affirmer une position finale, sur un ton indiscutable, même à propos de sujets mineurs.

\section{JOUER A LA HIÉRARCHIE}

S'accrocher à des positions de pouvoir formelles et leur donner plus d'importance qu'il ne faut.

\section{ÉVITER TOUTE EMOTION}

Intellectualiser, blaguer ou opposer une résistance passive lorsque vient le temps d'échanger des sentiments personnels.

\section{CONDESCENDANCE ET PATERNALISME}

Infantiliser les femmes et les nouveaux arrivants. Phrase typique : "maintenant, est-ce qu'une des femmes a quelque chose à ajouter ?"

\section{DRAGUER}

Traiter les femmes avec séduction, se servir de la sexualité pour les manipuler. "Humour" ambigu, pro-féminisme de façade. 
JOUER AU COQ

Aller chercher l'attention et le soutien des femmes en entrant en compétition avec les hommes face à elles.

ESTUDIANTITE AIGUE

Concentrer jalousement les informations-clé du groupe entre ses mains pour son propre usage et profit. 


\section{ANNEXE III}

\section{LISTE DES THÉMATIQUES DU JOURNAL CAUSE COMMUNE (JANVIER 2011)}

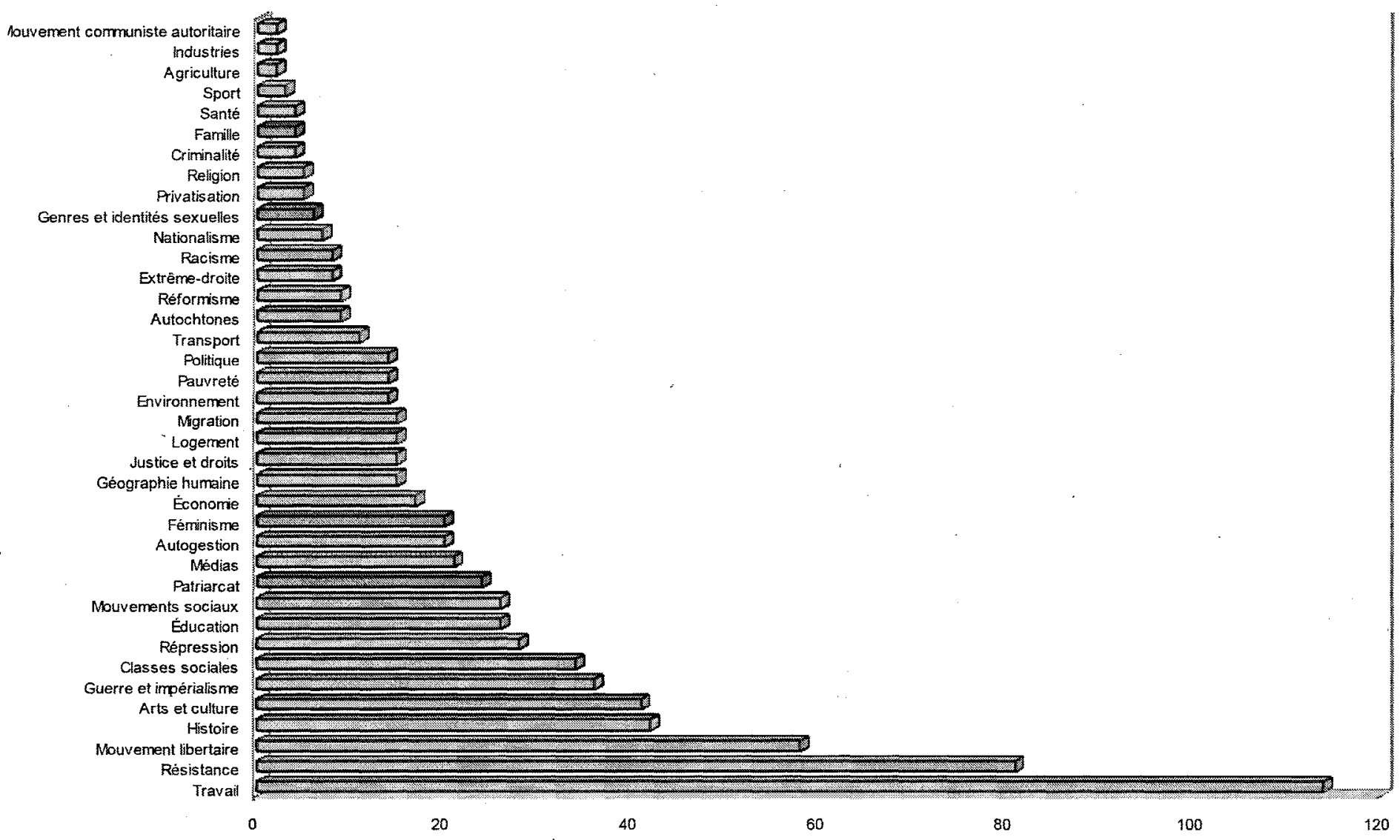

